\title{
Equilibrium and Nonequilibrium Behaviour of Ferrofluids - Experiments and Theory
}

\author{
By Jan Peter Embs ${ }^{1, *}$, Björn Huke ${ }^{2}$, Andreas Leschhorn², and \\ Manfred Lücke ${ }^{2}$ \\ ${ }^{1}$ Laboratory for Neutron Scattering ETH Zurich, Paul Scherrer Institut, \\ CH-5232 Villigen-PSI, Switzerland \\ ${ }^{2}$ Universität des Saarlandes, D-66041 Saarbrücken, Germany
}

(Received May 4, 2007; accepted August 1, 2007)

\section{Magnetic Fluids / Transport Properties / Pattern Formation}

Selected results on the spatiotemporal behaviour of equilibrium and nonequilibrium properties of ferrofluids in different magnetic fields are reviewed. They have been obtained in the project B13 Transport, response and instability behaviour of ferrofluids of the SFB 277 by experiments and by various theoretical methods ranging from purely analytical calculations to full numerical approaches.

\section{Introduction}

Ferrofluids are colloidal suspensions of permanent magnetic particles with magnetic moments of about $10^{4} \mu_{\mathrm{B}}$ in carrier liquids such as, e.g., oil or water. The small size of the particles in the range of about $10 \mathrm{~nm}$ provides stability against segregation due to gravity and common magnetic field gradients. To prevent aggregation due to van-der-Waals forces one customarily coats the particles by suitable surfactants.

Macroscopically, ferrofluids appear as paramagnetic liquids with a susceptibility $\chi \approx 1$ and higher, which is very large compared to molecular paramagnetic liquids. For this reason they are called super-paramagnetic. The large magnetic moments easily allow to exert significant magnetic forces and magnetic torques on the particles. This in turn has been stimulating not only a wealth of interesting basic research activities but also the development of many technical and medical applications: The forces and torques on the particles are mediated via the surfactant coating to the carrier liquid and with it to the ferrofluid. Thus, one can easily modify and control - on macroscopic

* Corresponding author. E-mail: jan.embs@psi.ch 
scales - the spatiotemporal properties of the equilibrium, transport, response and instability behaviour of the ferrofluid by magnetic fields.

Here we review selected results on the equilibrium as well as on the nonequilibrium behaviour of ferrofluids. They were obtained in the project B13 of the SFB 277 by experiments and by various theoretical methods ranging from purely analytical calculations to full numerical methods.

In Sect. 2 we present theoretical results concerning the equilibrium magnetization of monodisperse and polydisperse ferrofluids as obtained by BornMayer cluster expansion methods for dipolar hard spheres. The third section deals with a ferrofluid in a driven torsional pendulum undergoing small amplitude rotary oscillations close to resonance. With this setup one can measure among others the rotational viscosity of the ferrofluid in the absence of any macroscopic shear by enforcing the flow to be like a rigid body. Section 4 deals with a related problem, namely the relationship between magnetization and magnetic field in a ferrofluid that is rotating stationarily like a rigid body. Also here experiments as well as theoretical results are discussed and compared with each other. In Sect. 5 we investigate waves on the ferrofluid surface in vertically shaken containers in the presence of a magnetic field by experimental and numerical methods. In this experiment Faraday and Rosensweig instabilities of the flat ferrofluid surface can compete with each other. The final Sect. 6 presents a theoretical/numerical analysis of convection of ferrofluids in a Rayleigh-Bénard setup of a fluid layer heated from below. In particular the influence of the strong Soret effect and of the very slow concentration diffusion of the magnetic particles on the spatiotemporal behavior of convection is investigated.

\section{Equilibrium magnetization: Born-Mayer cluster expansion}

The most simple models of the microstructure of ferrofluids treat them as a gas of noninteracting, identical particles carrying a magnetic moment. The equilibrium magnetization in a magnetic field can then easily be found by analytical means and is described by a Langevin function.

However, the energy of the particle-particle interaction in ferrofluids can easily reach the order of $k T$ and therefore cannot simply be neglected anymore. Even if the particles can still be described as monodisperse, deviations of the magnetization curve from a Langevin curve will become apparent [1]. When particle-particle interaction is taken into account an analytical solution for the equilibrium magnetization is not possible anymore.

We used the Born-Mayer or cluster expansion technique to derive an approximate analytical expression describing the equilibrium magnetization of interacting ferrofluid particles [2,3]. This expression is given by the leading terms of an expansion in two relevant dimensionless parameters, namely the volume fraction $\phi$ of the magnetic material and an interaction parameter $\epsilon$ de- 
scribing the potential energy of a particle pair. The expansion is controlled. The calculated terms are exact for their respective orders in $\phi$ and $\epsilon$ within the model used, and deviations from the result for the equilibrium magnetization are thus of higher order in $\phi$ or $\epsilon$ than those calculated. We will describe the technique and the results in the next subsection.

Real ferrofluids are polydisperse, i.e. the particles have different sizes and magnetic moments. This fact alone leads to deviations from the Langevin behavior that can be as strong or stronger than the influence of the particleparticle interaction. A model of monodisperse interacting ferrofluid particles is thus in general not sufficient to explain the magnetization curves found in experiments and the effects of polydispersity $[4,5]$ have to be taken into account. We describe the necessary changes in the Born-Mayer expansion and the modifications of the theoretical results in the third subsection. In that section we also report applications of the theory to experimental magnetization curves.

\subsection{Monodisperse ferrofluids}

In monodisperse ferrofluids, calculating the equilibrium magnetization requires the evaluation of high-dimensional integrals of the form

$$
\int \exp \left(\sum_{i} \frac{\boldsymbol{m}_{i} \boldsymbol{H}_{e}}{k T}-\sum_{i<j} \frac{V_{i j}}{k T}\right) \mathrm{d} \boldsymbol{m}_{i} \mathrm{~d} \boldsymbol{x}_{i},
$$

that appear in the expression for the partition function. Here, $\boldsymbol{m}_{i}$ is the magnetic moment of the $i$-th particle at position $\boldsymbol{x}_{i}$ and $V_{i j}$ the interaction energy of the two particles $i$ and $j . \boldsymbol{H}_{e}$ is the applied magnetic field.

When $V_{i j}$ is ignored, the integral factorizes into one-particle terms and the analytical evaluation does not pose a problem. Within the Born-Mayer expansion terms $f_{i j}$ are introduced via

$$
\exp \left(-\frac{V_{i j}}{k T}\right)=1+f_{i j},
$$

allowing to rewrite (1) in a form

$$
\int \exp \left(\sum_{i} \frac{\boldsymbol{m}_{i} \boldsymbol{H}_{e}}{k T}\right) \prod_{i<j}\left(1+f_{i j}\right) \mathrm{d} \boldsymbol{m}_{i} \mathrm{~d} \boldsymbol{x}_{i} .
$$

When the typical interaction energy is small, the $f_{i j}$ are also small in most configurations.

The exponential function is now expanded into the $f_{i j}$, such that in the $n$-th order the high-dimensional integral (3) still factorizes, mostly into one-particle contributions. Only integrals over $n$ particles at maximum remain, allowing at least a numerical evaluation of the integrals. 
We modeled the interaction between the ferrofluid particles as dipoledipole-interaction $V^{\mathrm{DD}}$ plus a hard-sphere repulsion $V^{\mathrm{HS}}$ :

$$
V_{i j}=-\frac{3\left(\boldsymbol{m}_{i} \boldsymbol{r}_{i j}\right)\left(\boldsymbol{m}_{j} \boldsymbol{r}_{i j}\right)-r_{i j}^{2} \boldsymbol{m}_{i} \boldsymbol{m}_{j}}{4 \pi \mu_{0} r_{i j}^{5}}+V_{i j}^{\mathrm{HS}},
$$

$\boldsymbol{r}_{i j}$ being the distance vector between particle $i$ and $j$.

The carrier liquid is ignored in this treatment. That can safely be done if the interaction range of the carrier liquid's molecules is short compared to the typical distance of the ferro particles [6].

Making apart from the expansion in the $f_{i j}$ an additional expansion of the $\exp \left(-V_{i j} / k T\right)$ in powers of $V_{i j} / k T$, the leading terms of the equilibrium magnetization can even be evaluated analytically. In the final result the two expansions translate into expansions in the volume fraction $\phi=N \pi D^{3} / 6 \mathrm{~V}$ of the magnetic material and the interaction parameter $\epsilon=m^{2} / 4 \pi \mu_{0} k T D^{3}$, a typical interaction energy of two particles in contact (half the energy of a head-to-tail orientation). $D$ is the hard sphere particle diameter, $N / V$ the particle density. The expansion coefficients are functions of the dimensionless magnetic field $\alpha=m H / k T$ :

$$
M=M_{\mathrm{sat}}\left(\sum_{m, n} L_{m, n}(\alpha) \phi^{m} \epsilon^{n}\right) .
$$

$M_{\text {sat }}=N m / \mu_{0} V$ the saturation magnetization. The leading term $L_{0,0}$ is the Langevin function. There are no other terms with $m=0$ or $n=0$. The terms of first order in $\phi$ can in principle be calculated analytically in every order of $\epsilon$, only the integrands become more and more unwieldily. Using a computer algebra program we calculated the terms of order $\phi \epsilon^{n}$ up to $n=13$, an expansion that is useful if the ferrofluid is dilute but the particles possess a large magnetic moment. We also derived an analytical expression for the term of order $\phi^{2} \epsilon^{2}$, (the term of order $\phi^{2} \epsilon$ vanishes) which yields important contributions when the ferrofluid is highly concentrated.

The equilibrium magnetization as function of an external magnetic field depends also on the probe geometry, a consequence of the fact that dipolar forces decrease only $\propto r^{-3}$ and are therefore of long-ranged nature. Applying the thermodynamic limit $N \rightarrow \infty$ thus requires special care. In Eq. (1) the term of the outer potential contains correctly the magnetic field in absence of all other particles, i.e. simply the external field $\boldsymbol{H}_{e}$. To make the resulting expression for $\boldsymbol{M}$ independent of the geometry one has to enter the macroscopic internal field $\boldsymbol{H}$ into the calculation. To do so, one divides the dipolar interaction between the particles into those within a cutoff radius $R_{s}$ and those that are far away, i.e. beyond the cutoff. The long-ranging contributions within the sphere of radius $R_{s}$ cancel out and the limit $R_{s} \rightarrow \infty$ can thus safely be taken. The far-away particles are treated as a continuum that contributes a field $H_{s}=H+M / 3$ inside 
the sphere where $M$ is the sought after equilibrium magnetization. $H_{s}$ formally takes the role of $H_{e}$ in (1). The magnetization is then calculated using the selfconsistency condition $M=M\left(H_{s}\right)=M(H+M / 3)$ which can be solved in the required order to yield an analytical expression for $M(H)$ in the form given in Eq. (5).

The leading term in the expression for the reduced equilibrium magnetization $M / M_{\text {sat }}$ beyond the noninteracting approximation is of order $\phi \epsilon$. It has been explicitly considered in [7]. Furthermore, several other theories reproduce the correct $\phi \epsilon$-term when written in the form of a $(\phi, \epsilon)$-expansion [8-10]. However, no other theory also delivers the higher order terms correctly.

Taking the higher order functions $L_{m, n}(\alpha)$ into account significantly extends the range of validity of the formula (5). Deviations from the $\mathcal{O}(\phi \epsilon)$ approximation already become important when $\epsilon>0$ or $\phi \epsilon=0.04$. On the other hand, judging from the relative importance of the highest order terms we have calculated we expect our extended expression to fail at about $\epsilon=3$ and $\phi \epsilon=0.5$. This larger range is relevant for real ferrofluids: Volume fractions of about $\phi=0.5$ have been reported as well as dipolar interaction strengths of $\epsilon=2.7[11]$.

\subsection{Polydisperse ferrofluids}

Polydispersity has an influence on the magnetization curve already in the noninteracting case, and the influence on the interaction contributions is even stronger. To compare the results of the Born-Mayer expansion to experiments it is thus necessary to extend the theory to take polydispersity into account.

The necessary modifications are straightforward. In the integral (1) the absolute values $m_{i}$ of the magnetic particles will now be different, as well as the hard sphere diameters that enter into the interaction term $V_{i j}$. Sums over all $N$ particles are replaced by integrals $N \int P(m, D) \mathrm{d} m \mathrm{~d} D$ for a given distribution function $P(m, D)$ that can be replaced by a function $P(D)$ if a fixed relationship $m=m(D)$ is assumed. The result (5) now takes the form

$$
M=\overline{M_{\text {sat }}}\left(\sum_{m, n} L_{m, n}^{\mathrm{poly}}(\bar{\alpha}) \bar{\phi}^{m} \bar{\epsilon}^{n}\right),
$$

where the overlined quantities are now defined via "typical" values $\bar{D}$ and $\bar{m}$ for the particle diameter and magnetic moment. The functions $L_{m, n}^{\text {poly }}(\bar{\alpha})$ are polydisperse generalizations given by $m$-fold integrals over the reduced diameters $D_{i} / \bar{D}$ and magnetic moments $m_{i} / \bar{m}$.

For reasons of simplicity we will assume the definitions $\bar{D}^{3}=\left\langle D^{3}\right\rangle$ and $\bar{m}=\langle m\rangle$ which unambiguously defines $L_{m, n}^{\text {poly }}$ if $P(m, D)$ is given. The reference values $\bar{D}$ and $\bar{m}$ can in fact be chosen freely however, which facilitates the application of the theory in cases where the actual distribution function might not be known beforehand, e.g. in magneto-granulometric measurements. 


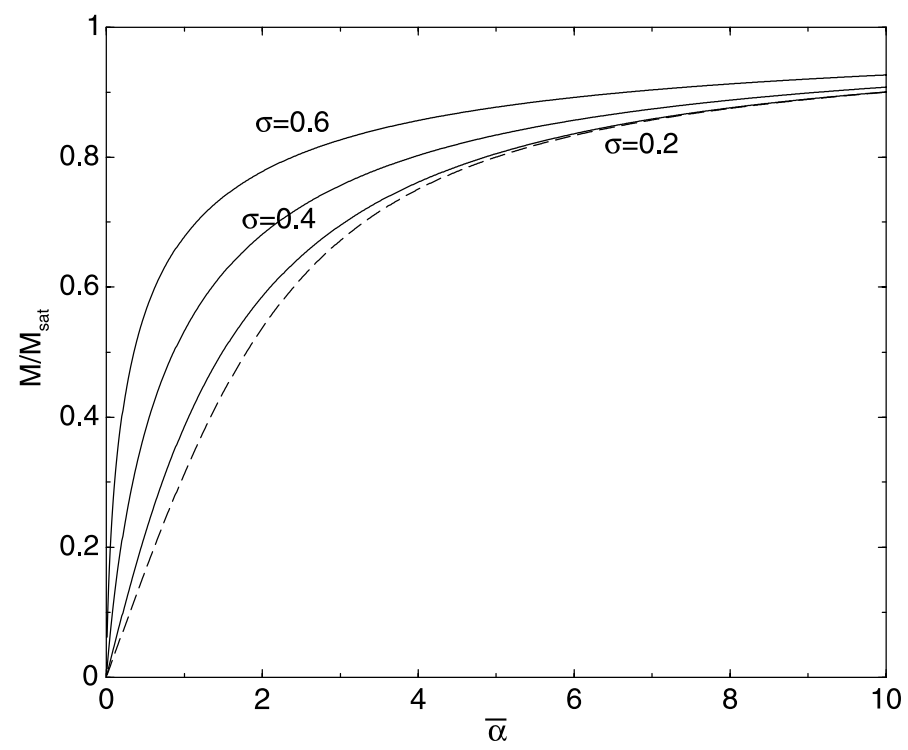

Fig. 1. Magnetization curves for noninteracting ferrofluids with the same mean magnetic moment but different distribution widths. The dashed line is the monodisperse result.

\subsubsection{Results}

The influence of polydispersity becomes apparent in Fig. 1 where we have plotted the magnetization curves for several ferrofluids with a lognormal size distribution $P(D) \sim \mathrm{e}^{-\log \left(D / D_{0}\right)^{2} / 2 \sigma^{2}}$. The variance $\sigma$ of $\log D$ assumes typical values found in experiment and $D_{0}$ is chosen such that $\langle m\rangle \sim\left\langle D^{3}\right\rangle$ is the same for all curves. The particle-particle interaction is not taken into account. A finite width of the distribution leads to an increased magnetization for medium fields, the same qualitative effect as for the particle-particle interaction. To discriminate between both effect in experiments further knowledge about the ferrofluid than just the magnetization curve is needed. We will discuss this in more detail in Sect. 2.2.2. The influence of polydispersity on the higher-order interaction terms $L_{m, n}^{\text {poly }}$ is even larger since they depend on higher order moments of the $m$ - and $D$-distribution which grow with the width of the distribution even if lower order moments are kept fixed.

This growing importance of the higher order terms in $\bar{\phi}$ and $\bar{\epsilon}$ makes all finite order approximations less reliable than in the monodisperse case, at least for distributions with a long tail. The situation differs for distributions with a realistic cutoff. Figure 2 shows the result of our theory for a bidisperse system. The relative particle density of one of the components is plotted against the initial susceptibility. The two particle species have a volume ratio of $1: 2$ and the 


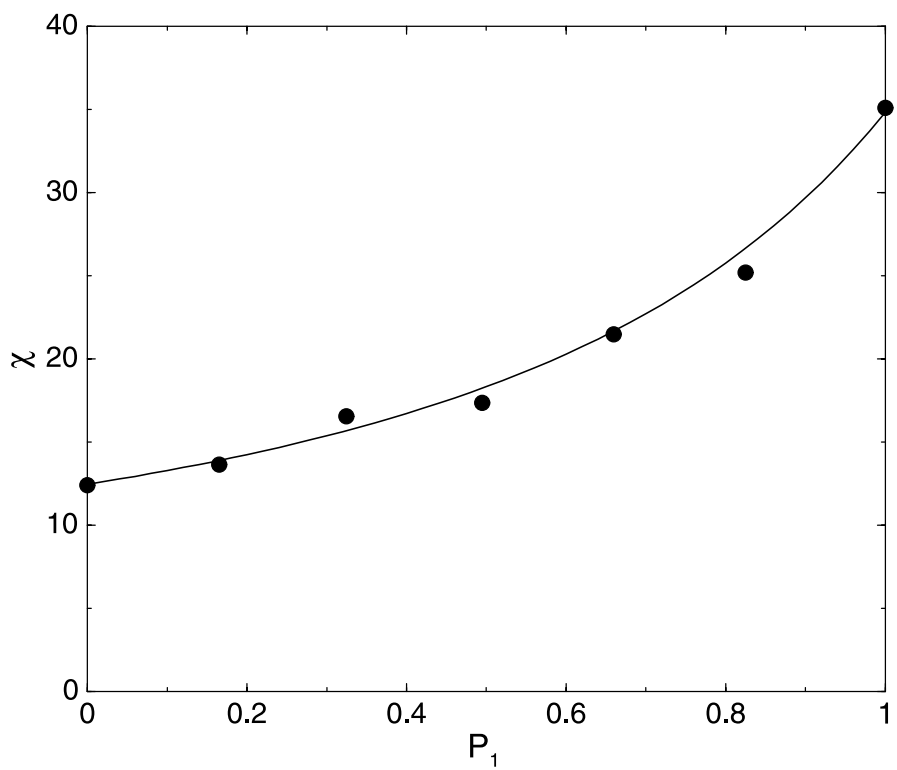

Fig. 2. The initial susceptibility of a bidisperse ferrofluid as described in the text. $P_{1}$ is the relative volume fraction of one of the species. The solid line is the result of the cluster expansion theory, the dots are numerical results by Cummings and Blum [12].

volume fraction of both species together is $\phi=0.42$. The smaller species has a larger magnetic moment such that $\epsilon=2.24$ for this species whereas $\epsilon=1.34$ for the larger species. The comparison to Monte Carlo data by Cummings and Blum [12] who have considered this system before shows a very good agreement.

\subsubsection{Comparison to experiments}

Since polydispersity can mimic the influence of dipolar interaction and vice versa, testing the cluster expansion theory in experiments requires some additional knowledge apart from a single magnetization curve.

We have applied the theory to experimental data on the equilibrium magnetization of the ferrofluid APG 933 by Ferrotec. The diameter distribution was taken from TEM measurements. Figure 3 shows the results for the diameter distribution (lower plot) as well as the experimentally found equilibrium magnetization (points, upper plot) versus the predictions of our theory (solid curve, upper plot). Apart from the diameter distribution only known quantities such as the saturation magnetization of magnetite and the ferrofluid itself, as well as reasonable assumptions about the structure of the particles, in particular the thickness of the polymer layer and the magnetically dead layer [13] entered 

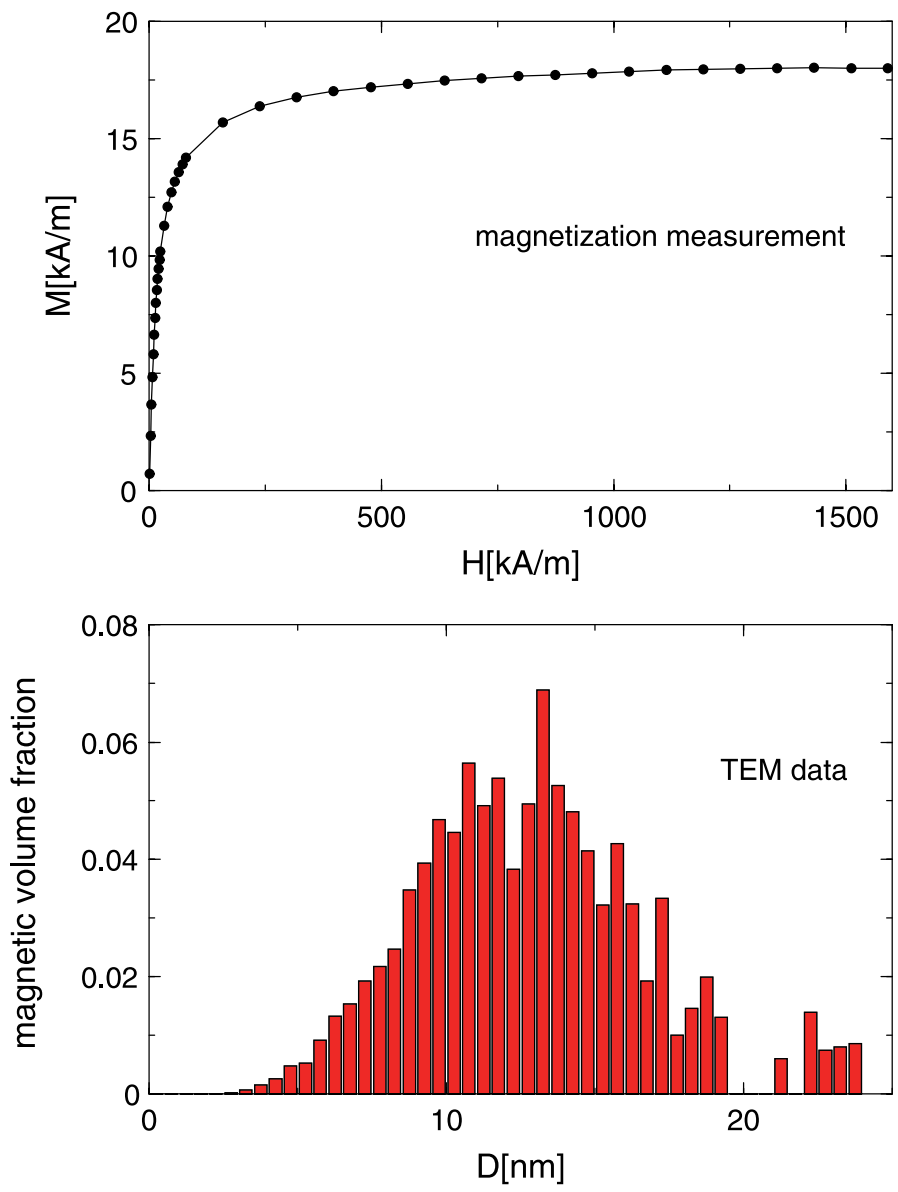

Fig. 3. Equilibrium magnetization of the ferrofluid APG 933 as measured in experiments (upper plot, points) versus the results of our theory (solid line) for the diameter distribution found in TEM measurements (lower plot).

the calculation. No fit parameters were required. Nevertheless the agreement between theoretically and experimentally found magnetization curves is excellent.

We also applied our theory to magnetization curves of a series of ferrofluids prepared by Kuznetsova and Ivanov via diluting a highly concentrating basic ferrofluid. In this case the diameter distribution $P(D)$ was a priori unknown. Assuming a $\Gamma$-distribution $P(D) \sim D^{\beta} \mathrm{e}^{-D / D_{0}}$ the task of finding $P(D)$ can be reduced to determining two fit parameters $\beta$ and $D_{0}$. Under the condition that the dilution process does not change $P(D)$, different models for the equilibrium magnetization can be judged by comparing the fit results for the different sam- 
ples. The results for the volume weighted mean diameter (in $\mathrm{nm}$ ) are shown in the following table:

\begin{tabular}{lccccccc}
\hline Degree of dilution: & 0.088 & 0.137 & 0.197 & 0.296 & 0.444 & 0.664 & 1 \\
\hline Langevin & 7.1 & 7.2 & 7.0 & 6.4 & 6.3 & 6.3 & 6.0 \\
Weiss [8] & 7.3 & 7.6 & 7.7 & 7.6 & 8.0 & 8.5 & 8.9 \\
MSM [9] & 7.3 & 7.5 & 7.5 & 7.1 & 7.2 & 7.2 & 6.9 \\
HTA [7] & 7.3 & 7.4 & 7.4 & 6.9 & 6.7 & 6.5 & 5.6 \\
MMFM-1 [10] & 7.3 & 7.5 & 7.5 & 7.1 & 7.1 & 7.1 & 6.8 \\
CET [5] & 7.13 & 7.13 & 7.13 & 7.19 & 7.24 & 7.46 & 8.25 \\
MMFM-2 [14] & 7.30 & 7.32 & 7.32 & 7.31 & 7.30 & 7.30 & 7.29 \\
\hline
\end{tabular}

Our cluster expansion theory (CET) showed the second most consistent results for the mean volume and only fails for the highest concentrations where the second order modified mean field model by Ivanov [14] turned out to be best. The results for the variance that are not shown here are similar. The shortcoming of the cluster expansion here is probably due to the strong contribution of the higher order $\phi \epsilon^{n}$-terms. In Ivanov's theory such terms are not taken into account at all. The small differences for the fit results of our theory compared to all others already at the smallest concentrations is due to the fact that we used a binned approximation to the $\Gamma$-distribution for $P(D)$ instead of a smooth curve. This also demonstrates that the calculated means and variances may depend sensitively on the model distribution used.

To conclude: Using the Born-Mayer or cluster expansion technique we have derived a formula for the equilibrium magnetization given by the leading terms of a controlled expansion in two parameters $\phi$ and $\epsilon$ modelling the ferrofluid as a system of dipolar hard spheres. We also derived a polydisperse generalization that allows to calculate the magnetization for arbitrary distributions of magnetic moments and hard sphere diameters. The formulas have been tested on both data from numerical simulations and experimental data.

\section{Resonantly driven torsional pendulum in a magnetic field}

A ferrofluid in solid-body rotation experiences a viscous dissipation if an external magnetic field is applied perpendicular to the rotation axis. This so-called magneto-viscous effect [15] is attributed to a field dependent "rotational viscosity" $\eta_{\mathrm{R}}$. With a magnetic torque acting on each magnetic colloidal particle its rotation in phase with the local vorticity of the carrier liquid is impeded thus giving rise to dissipative losses. In a mesoscopic picture the latter are due to friction between the particles and the carrier liquid. Now, the velocity gradient tensor of an arbitrary macroscopic incompressible flow can be decomposed into antisymmetric contributions (vorticity) and a traceless symmetric tensor 
reflecting shear (or, more precisely, elongational flow). In ordinary fluids only the latter contributes to dissipation proportional to the shear viscosity $\eta$ so that a solid-body rotation remains undamped there.

Thus, a ferrofluid flow in which rotation as well as shear is present poses the theoretical and experimental problem of extracting from the total dissipation the contribution due to $\eta_{\mathrm{R}}$. The main advantage of our experimental setup is that it ensures at every instant of the measurement a rigid rotation of the fluid without disturbing shear. Then the genuinely rotational dissipation rate of the ferrofluid is measured in a resonance experiment by a high quality factor torsional pendulum that is forced harmonically close to resonance. The rotational viscosity $\eta_{\mathrm{R}}$ is related to the transversal magnetic relaxation time $\tau_{\perp}$. By fitting the experimental result for $\tau_{\perp}$ to a simple mesoscopic model we deduce the average size of the magnetic core and of the hydrodynamic diameter of the suspended particles [16-18].

Finally we investigate analytically the effect of polydispersity on the dynamic response behavior of the ferrofluid pendulum.

\subsection{Comparison with other characterization methods}

In our experiments commercially available magnetite-based ferrofluids, (APG 933-936 FerroTec, Nürtingen), and a sample composed of cobalt particles (Kardiotechnik, Berlin) were investigated. The magnetite grains of the APG series are coated with a tenside layer and suspended in a hydrocarbon carrier liquid. The individual sample fluids of the APG series differ in their respective viscosities.

The Co particles were coated by lauryol-sarcosine and dispersed in a diffusion pump oil (Edwards L9). A thickener agent was added to enhance the viscosity of the sample. A dilution series (to ratios as low as $1: 2000$ ) of the Cobased ferrofluid, necessary for the light-scattering experiments and the TEM analysis, was prepared by adding carrier oil. From measurements of the saturation magnetization, we estimated the volume fraction of cobalt in the undiluted samples to be $\varphi=1.5 \%$. The specifications of the ferrofluid samples used are summarized in Table 1.

\subsubsection{Magneto-granulometry $(M G)$}

The distribution $g(D)$ of ferromagnetic core diameters $D$ in a particular sample can be derived from the equilibrium magnetization curve $M_{\mathrm{eq}}$ in an external field $H_{\mathrm{ext}}$. In a dilute sample of monodisperse ferromagnetic colloids (i.e. one in which dipolar interactions between particles can be neglected), one expects the magnetization to follow the Langevin behavior of an ideal dipolar gas, which has the form $M_{\mathrm{eq}}=M_{\mathrm{s}} \mathcal{L}(\alpha)$, where $M_{\mathrm{s}}$ is the saturation magnetization, $\mathscr{L}(\alpha)$ denotes the Langevin function $\operatorname{coth}(\alpha)-1 / \alpha$, and $\alpha$ is the Langevin parameter $m H_{\text {ext }} /\left(k_{\mathrm{B}} T\right)$, with particle magnetic moment $m=\mu_{0} \pi D^{3} M_{d} / 6$. Here, $\mu_{0}$ denotes the vacuum permeability, $M_{d}$ the bulk 
Table 1. Specifications of the investigated ferrofluids. The volume fraction $\varphi$ of the ferromagnetic material is deduced from the measured saturation magnetization $M_{\mathrm{s}}$ using the relation $\varphi=M_{\mathrm{s}} / M_{\mathrm{B}}$, where $M_{\mathrm{B}}$ is the tabulated value for the bulk magnetization of the ferromagnetic material at room temperature $\left(M_{\mathrm{B}}=4.46 \times 10^{5} \mathrm{~A} / \mathrm{m}\right.$ for magnetite and $14.01 \times 10^{5} \mathrm{~A} / \mathrm{m}$ for cobalt). The quantity $\chi_{i}$ is the magnetic susceptibility, as determined from the initial slope of $M v s$. $H$, and $\eta$ is the dynamic viscosity, as provided by the manufacturers.

\begin{tabular}{lcccc}
\hline Ferrofluid & $M_{\mathrm{s}}(\mathrm{A} / \mathrm{m})$ & $\varphi(\%)$ & $\chi_{i}$ & $\eta(\mathrm{Pa} \mathrm{s})$ \\
\hline APG 933 & 18139 & 4.1 & 1.09 & 0.5 \\
APG 934 & 16398 & 3.7 & 0.73 & 1.0 \\
APG 935 & 17885 & 4.0 & 0.58 & 1.5 \\
APG 936 & 19010 & 4.3 & 0.85 & 2.0 \\
Co & 6512 & 1.5 & 0.44 & 0.5 \\
\hline
\end{tabular}

magnetization of the magnetic material, $k_{\mathrm{B}}$ the Boltzmann constant and $T$ the temperature.

In a real ferrofluid the polydispersity of the colloid and the interparticle interactions lead to noticeable deviations from ideal Langevin behavior [19]. The polydispersity can be approximated by assuming a lognormal distribution of particle sizes:

$$
g(D)=\frac{1}{\sqrt{2 \pi} D \ln \sigma} \exp \left[-\frac{\ln ^{2}\left(D / D_{0}\right)}{2 \ln ^{2} \sigma}\right],
$$

where $D_{0}$ is the median and $\sigma$ the geometric standard deviation of the distribution. If the dipolar coupling is not too strong and the concentration $\varphi$ not too high, the dipolar particle interaction can be approximated within the mean-field Weiss model [19]. To that end the external field $H_{\text {ext }}$ appearing in the Langevin parameter $\alpha$ is replaced by the local magnetic field $H_{\text {loc }}=H+(M / 3)$, where $M / 3$ is the Lorentz cavity field $[20,21], H=H_{\text {ext }}-\bar{N} M$ is the macroscopic field inside the sample, and $\bar{N} M$ is the demagnetization related to the sample geometry. For our sample geometry we have $H_{\mathrm{loc}} \simeq H_{\mathrm{ext}}-0.11 \mathrm{M}$.

The equilibrium magnetization $M_{\text {eq }}$ can then be written in the following integral representation

$$
M_{\mathrm{eq}}=M_{\mathrm{s}} \frac{\int_{0}^{\infty} x^{3} g(x) \mathcal{L}\left(\mu_{0} M_{\mathrm{d}} \frac{\pi x^{3}}{6 k_{\mathrm{B}} T} H_{\mathrm{loc}}\right) \mathrm{d} x}{\int_{0}^{\infty} x^{3} g(x) \mathrm{d} x} .
$$

We recorded the magnetization of the ferrofluid samples using a vibratingsample magnetometer (Lake Shore VSM, Type 7300). The dashed line in Fig. 4 is a pure Langevin fit to such a magnetization curve, indicating that a monodisperse particle distribution $(\sigma=1)$ is a rather poor approximation for the true particle-size distribution. A lognormal distribution of particle 


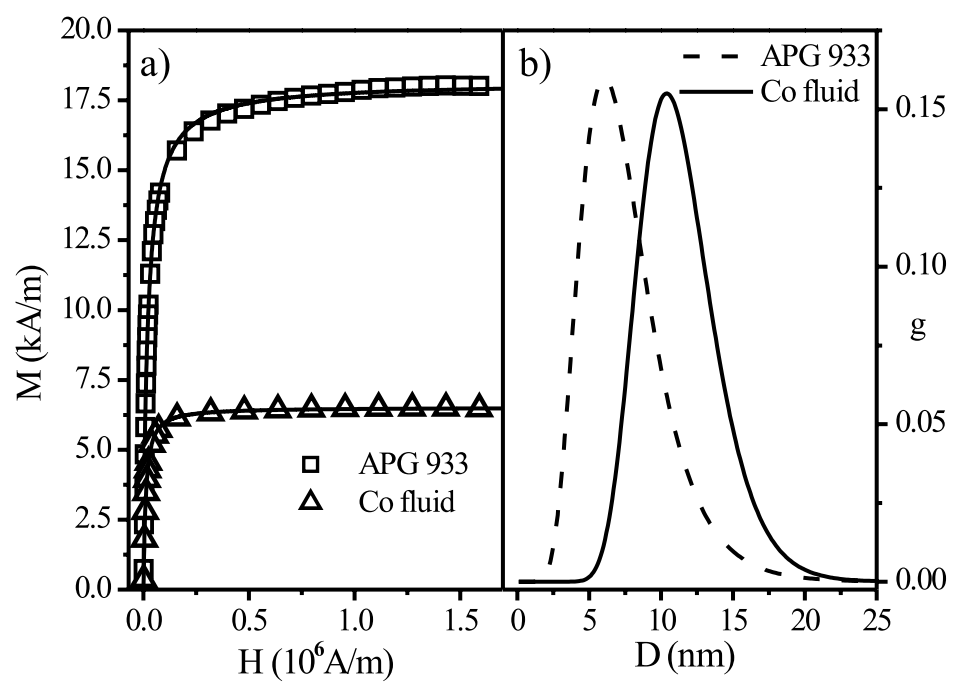

Fig. 4. Symbols denote the measured equilibrium magnetization for APG 933 as a function of the macroscopic magnetic field $H$ within the sample. The solid line is a leastsquares fit according to Eq. (8) with $D_{0}, \sigma$, and $M_{\mathrm{s}}$ as given in Table 2. The dashed line is a fit for a monodisperse ferrofluid (Langevin behavior) with $D=11.1 \mathrm{~nm}$ and $M_{\mathrm{s}}=17563 \mathrm{~A} / \mathrm{m}$.

diameters, however, results in close agreement between measured and calculated magnetization curves (Fig. 4). The lognormal distribution parameters obtained from magneto-granulometry analyses of the ferrofluid samples are listed in Table 2. Additionally, Table 2 contains values for the volume-averaged diameter $D_{v}=\left\langle D^{3}\right\rangle^{1 / 3}=D_{0} \exp \left[(3 / 2) \ln ^{2} \sigma\right]$, which can be measured by the torsional-pendulum method (Sect. 3.2), and for the volume-weighted average diameter $D_{\text {vol }}=\left\langle D^{4}\right\rangle\left\langle D^{3}\right\rangle=D_{0} \exp \left[(7 / 2) \ln ^{2} \sigma\right]$, which is the size parameter customarily provided by X-ray diffractometry (Sect. 3.1.2).

To rule out a possible concentration dependence of these parameters, we investigated a dilution series with n-heptane ranging from the non-diluted volume concentration of $\varphi \simeq 3.3 \%$ down to concentrations as low as $0.019 \%$. From the scatter in the parameter values, we estimate the uncertainties associated with the magneto-granulometric size determination to be $\pm 1.0 \mathrm{~nm}$ for $D_{0}$ and \pm 0.02 for $\sigma$.

\subsubsection{X-ray diffraction $(X R D)$}

Using XRD, we determined the volume-weighted average core size of the ferrofluid particles. A glass substrate holder was filled with undiluted ferrofluid and mounted in a Siemens D-5000 diffractometer with $\theta-\theta$ geometry. Wideangle diffraction scans were recorded from $10^{\circ}$ to $140^{\circ}(2 \theta)$ with a typical step 
Table 2. The core-size distribution parameters $D_{0}$ and $\sigma$ and the saturation magnetization $M_{\mathrm{s}}$ for the ferrofluid samples, as determined by magneto-granulometry using Eq. (8). Also listed are the volume-averaged core diameter $D_{v}=\left\langle D^{3}\right\rangle^{1 / 3}$ and the volume-weighted core diameter $D_{\text {vol }}=\left\langle D^{4}\right\rangle /\left\langle D^{3}\right\rangle$.

\begin{tabular}{lccccc}
\hline Ferrofluid & $M_{\mathrm{s}}(\mathrm{A} / \mathrm{m})$ & $D_{0}(\mathrm{~nm})$ & $\sigma$ & $D_{v}(\mathrm{~nm})$ & $D_{\text {vol }}(\mathrm{nm})$ \\
\hline APG 933 & 18149 & 7.0 & 1.47 & 8.7 & 11.7 \\
APG 934 & 16400 & 7.1 & 1.42 & 8.5 & 10.9 \\
APG 935 & 17886 & 6.3 & 1.44 & 7.6 & 9.7 \\
APG 936 & 19013 & 7.0 & 1.42 & 8.4 & 10.8 \\
Co & 6512 & 11.0 & 1.27 & 12.0 & 13.5 \\
\hline
\end{tabular}

size of $0.02^{\circ}$ and count time of $20 \mathrm{~s}$ using $\mathrm{Cu} K_{\alpha}$ radiation $(\lambda=1.54 \AA)$. All Bragg peaks recorded in the diffraction measurements of the ferrofluid samples APG 933-936 could be indexed to magnetite $\left(\mathrm{Fe}_{3} \mathrm{O}_{4}\right)$; no evidence for the presence of secondary phases was found. The Bragg peaks were broadened considerably with respect to the peak widths expected for diffraction from a coarse-grained $(>1 \mu \mathrm{m})$, strain-free sample of magnetite. This indicates the presence of a significant amount of inhomogeneous lattice strain and/or a broad size distribution of the magnetite cores, with an average size well below $50 \mathrm{~nm}$ [22]. By performing a standard peak-profile analysis according to the method of Warren and Averbach [23-26] or a full-pattern profile fit [27], it is possible to separate the lattice-strain effect from that of the crystallite size and thereby determine the inhomogeneous strain level $e$ and the volume-weighted average crystallite size $D_{\text {vol }}$. Furthermore, one can also estimate the geometric standard deviation $\sigma$ of the lognormal distribution of spherical crystallites that would give rise to Bragg peak profiles most nearly identical to those actually measured [27].

The result of a full-pattern profile fit to the diffraction scan of sample APG 936 is shown in Fig. 5. This fitting technique simultaneously refines $D_{\text {vol }}, \sigma$, $e$, the lattice parameter of magnetite and a polynomial background function in order to obtain a least-squares fit to the measured diffraction intensity.

In Table 3 the results of full-pattern profile fits to the four APG ferrofluid specimens are collected. Within the uncertainty range of this analysis technique $( \pm 10 \%)$, the crystallite sizes and lattice strains were found to be identical for all samples. Averaging over the indicated values, we find $D_{\text {vol }}$ equal to (11.8 \pm $0.7) \mathrm{nm}$ and $\sigma$ equal to $1.58 \pm 0.04$. Averaging over the inhomogeneous latticestrain values yields $e=(0.29 \pm 0.05) \%$.

Comparing the size distributions obtained by XRD with those of MG, we observe that the $D_{0}$ values from XRD are systematically smaller (on average by $20 \%$ ), and the width parameter $\sigma$ is larger by about $8 \%$. However, the results of the two methods are consistent within the ranges of experimental uncertainty for $D_{0}$ and $\sigma$. The agreement between the values obtained by each method for 


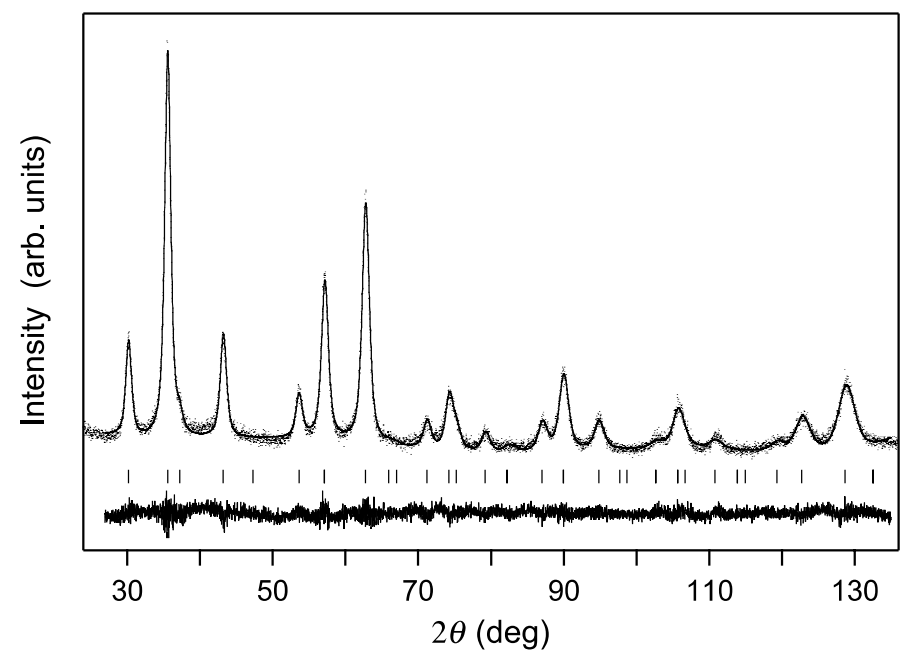

Fig. 5. X-ray diffraction scan of ferrofluid APG 936 (dots). The solid curve superimposed on the measured data is the result of a full-pattern profile fit between $27^{\circ}$ and $135^{\circ}$; below that lies the difference curve between the measured and calculated diffraction intensities. Vertical lines indicate the expected positions of Bragg reflections from $\mathrm{Fe}_{3} \mathrm{O}_{4}$. The fit curve represents the calculated diffraction intensity arising from a lognormal distribution of spherical crystallites with a median diameter of $D_{0}=5.3 \mathrm{~nm}$ and a geometric standard deviation of $\sigma=1.60$.

Table 3. The parameters $D_{0}$ and $\sigma$ of a lognormal distribution of crystallite sizes, as obtained from XRD analysis of the magnetite-based ferrofluids. Also included are the volume-averaged diameter $D_{v}=\left\langle D^{3}\right\rangle^{1 / 3}$ and the volume-weighted average diameter $D_{\text {vol }}=\left\langle D^{4}\right\rangle /\left\langle D^{3}\right\rangle$.

\begin{tabular}{ccccc}
\hline Ferrofluid & $D_{0}(\mathrm{~nm})$ & $\sigma$ & $D_{v}(\mathrm{~nm})$ & $D_{\text {vol }}(\mathrm{nm})$ \\
\hline APG 933 & 5.4 & 1.64 & 7.8 & 12.7 \\
APG 934 & 6.3 & 1.54 & 8.4 & 12.1 \\
APG 935 & 5.7 & 1.53 & 7.5 & 10.7 \\
APG 936 & 5.3 & 1.60 & 7.3 & 11.5 \\
\hline
\end{tabular}

the volume-averaged diameter $D_{v}$ is even better, with a mean difference of less than $7 \%$.

\subsubsection{Transmission electron microscopy (TEM)}

Samples for TEM analysis were prepared by diluting the magnetite-based ferrofluid samples with n-heptane in an ultrasonic bath and then transferring a small amount to copper grids coated with amorphous carbon. Bright- 
field/dark-field image pairs and electron-diffraction patterns were recorded at $200 \mathrm{kV}$ on a JEOL 2010 microscope. Cryo-TEM investigations were performed on APG 933, comparing an undiluted sample $(\varphi=4.1 \%)$ with a diluted one $(\varphi=0.007 \%)$. The cryo-TEM studies-performed by the base-grid method-entailed shock-freezing the sample holder carrying the ferrofluid before introducing it into the TEM. In addition, a high-resolution TEM analysis was carried out on individual magnetite particles in highly diluted specimens.

Unlike XRD scans, dark-field TEM images provide a direct measure for the spatial extent of the individual crystallites in a sample (see inset in Fig. 6). From such images one can determine the crystallite-size distribution without having to rely on an assumed distribution shape, as is the case with XRD or MG. Histograms approximating the crystallite-size distribution in the ferrofluid samples were generated by computer-assisted analysis of several dark-field images (Fig. 6). The resulting distribution can indeed be closely approximated by a lognormal function, as was assumed in the XRD and MG analyses. Furthermore, the corresponding values for $D_{0}$ and $\sigma$ are very close to those obtained by XRD and MG.

To investigate the possible occurrence of particle agglomeration caused by diluting the sample with $\mathrm{n}$-heptane or subsequent evaporation of heptane from the sample while in the microscope, we performed cryo-TEM investigations of a series of ferrofluids of varying concentration. Figure 6 shows the images of an undiluted (a) and highly diluted (b) sample of APG 936. The associated crystallite-size histograms shown in (c) do not differ significantly, indicating that there is no measurable particle agglomeration.

Figure 6a also sheds light on the hydrodynamic diameter of the particles in the APG 936 sample. Taking the undiluted volume fraction of $\varphi=4.3 \%$ and the measured particle core diameter of $D_{v} \simeq 8.4 \mathrm{~nm}$ (Table 1 and Table 2), one obtains for the average light distance between the surfaces of adjacent particles $11 \mathrm{~nm}$. However, the analysis of Fig. 6a (also performed on the undiluted sample) reveals that the particles are able to approach closer to each other. Here the evaluated average light distance is only $(5.2 \pm 1.7) \mathrm{nm}$. We take half of this value as an upper limit for the thickness of the particle coating.

\subsubsection{Photon-correlation spectroscopy (PCS)}

Dynamic light-scattering experiments performed on diluted ferrofluid samples yield a value for the diffusion coefficient of the particles, which in turn depends on the hydrodynamic particle diameter $D^{\text {hyd }}$. In our studies, PCS was carried out on an ALV 5000 spectrometer equipped with a krypton-ion laser (Spectra Physics). Dilution series were investigated with volume fractions ranging from $\varphi=0.002 \%$ to $0.02 \%$. The intensity correlation function of the scattered light was recorded with an ALV-5000E correlator. Thereafter, the data were 

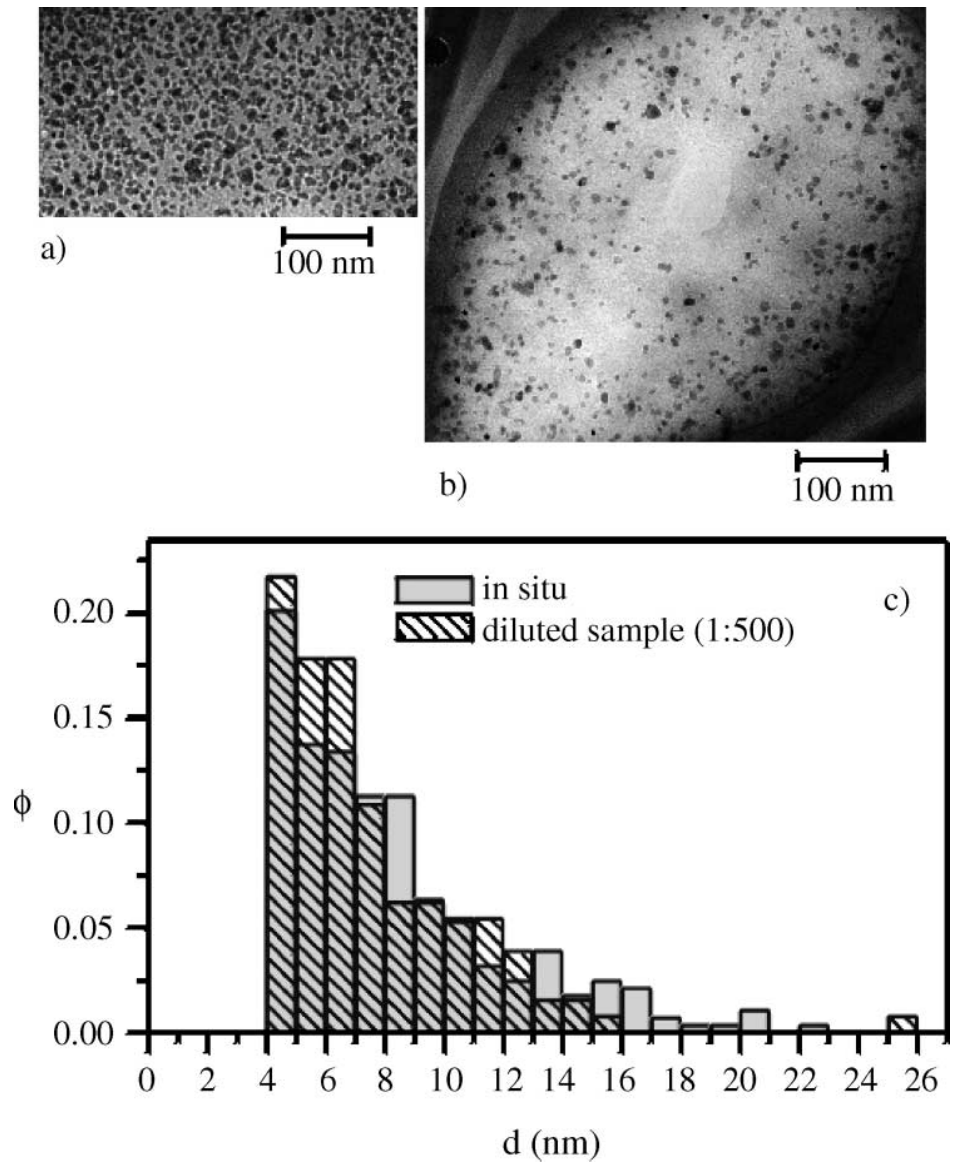

Fig. 6. Crystallite-size histograms (c) obtained by cryo-TEM studies of an undiluted sample (a) of APG 936 (grey histogram bars) and after strong dilution (b) with n-heptane to $\varphi=0.0067 \%$ (striped histogram bars).

fitted using the ALV-NonLin routine, which is based on the CONTIN regularization [28]. This analysis yields an intensity-weighted distribution function for the decay times. Under the assumption that the scattering particles behave like hard spheres in dilute solution, one can deduce the distribution of the hydrodynamic radii from the Stokes-Einstein relation. Applying an appropriate data weighting, one then obtains the number-weighted hydrodynamic-diameter distribution function $g\left(D^{\text {hyd }}\right)$ from the intensity-weighted distribution.

Figure 7 illustrates the distribution $g\left(D^{\text {hyd }}\right)$ for the magnetite-based ferrofluid APG 934 and for the Co-based sample. By fitting such CONTIN curves with a lognormal distribution (dashed lines), we obtain the size parameters $D_{0}$ and $\sigma$, which can be compared directly to the results of the other sample 


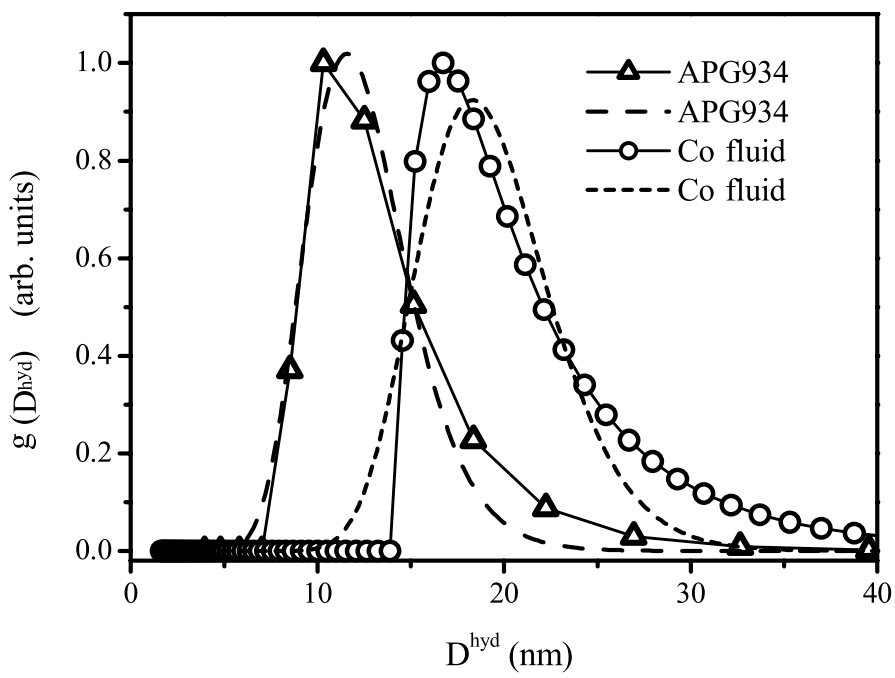

Fig. 7. Number-weighted distribution $g\left(D^{\text {hyd }}\right)$ for the hydrodynamic diameter, as obtained from the CONTIN regularization of the intensity correlation. Fitting the curves with a lognormal distribution (dashed lines) yields the parameters $D_{0}$ and $\sigma$ listed in Table 4.

Table 4. The parameters $D_{0}$ and $\sigma$ of a lognormal distribution of hydrodynamic diameters, as obtained from PCS measurements. Values for the volume-averaged hydrodynamic diameter $D_{v}=\left\langle D^{3}\right\rangle^{1 / 3}$ and the volume-weighted average hydrodynamic diameter $D_{\text {vol }}=\left\langle D^{4}\right\rangle /\left\langle D^{3}\right\rangle$ are also listed.

\begin{tabular}{lcccc}
\hline Ferrofluid & $D_{0}(\mathrm{~nm})$ & $\sigma$ & $D_{v}^{\text {hyd }}(\mathrm{nm})$ & $D_{\text {vol }}^{\text {hyd }}(\mathrm{nm})$ \\
\hline APG934 & 12.1 & 1.2 & 12.7 & 14.0 \\
APG935 & 14.5 & 1.2 & 15.2 & 16.8 \\
APG936 & 18.6 & 1.2 & 19.5 & 21.6 \\
Cobalt & 19.0 & 1.2 & 20.0 & 22.0 \\
\hline
\end{tabular}

characterizations. The resulting size parameters are listed in Table 4 together with the mean values $D_{v}^{\text {hyd }}$ and $D_{\text {vol }}^{\text {hyd }}$. From the scatter in measurements made at different angles, we estimate the uncertainty of the mean particle size to be $\pm 1.5 \mathrm{~nm}$.

\subsection{Measurements of the rotational viscosity}

One of the many fascinating properties of ferrofluids [15] is their tunable viscosity which can be controlled by an external magnetic field. This so called magneto-viscous effect has first been observed by McTague [29]. Qualitatively it can be accounted for [30] by magnetic torques acting upon the suspended 
ferromagnetic particles: depending on the relative orientation between magnetic field and local vorticity the particle rotation is hindered. The friction at the coated particle surfaces generates an extra dissipation and thus leads to an enhanced effective viscosity. This additional rotational friction is described by a "rotational viscosity" $\eta_{\mathrm{R}}$. Based on the theory of rotary Brownian motion of non-interacting rigid dipoles, Shliomis [31] derived an analytic expression for the magnetic field dependence of $\eta_{\mathrm{R}}$.

We have measured this field induced magneto-viscous damping by means of a torsional pendulum of a high quality factor [32]. While the setup is straightforward and relatively simple it does not seem to have been used before to measure $\eta_{\mathrm{R}}$ [33]. Yet it bypasses in an elegant way problems ( $c f$. our further discussion below) that arise in earlier experiments [21, 29, 34-37]. Therein the effects of shear- and of rotational friction become mixed up. Moreover, one has to invoke partly untested theoretical assumptions to extract the relatively small (only a few percent) rotational contribution. The main advantage of our setup is to ensure at every instance a solid-body rotation of the ferrofluid without dissipative shear. The rotational viscosity, i.e., the dissipation arising by internal viscous friction between magnetic particles and carrier fluid is then quantified by determining the damping rate of the pendulum.

\subsubsection{System and experimental procedure}

In our experiment a cylindrical Plexiglas container (inner height $15.1 \mathrm{~mm}$, diameter $16 \mathrm{~mm}$ ) filled with the ferrofluid APG 933 (Ferrofluidics) [38] is mounted on the carrier platform of a torsional pendulum (Fig. 8). The moment of inertia $\Theta$ of the oscillating body was determined by measuring the resonance frequencies without payload and with a payload of known moment of inertia. $\Theta$ is dominated by the mass of the carrier platform. At zero magnetic field the resonance frequency of the pendulum with the ferrofluid is $\Omega_{0} / 2 \pi=32.7 \mathrm{~Hz}$ and the damping rate is $\Gamma_{0}=0.178 \mathrm{~Hz}$ giving a quality factor of $\Omega_{0} / \Gamma_{0} \simeq 1150$. Capacitive driving is realized at one arm of the carrier with a high voltage ac-source of tunable frequency $f=\omega / 2 \pi$. A second similar capacitor at the other carrier arm is connected to a lock-in amplifier and records the deflection angle $\varphi(t)$. Its amplitude was always below $1^{\circ}$. A static homogeneous external magnetic field $\boldsymbol{H}^{\text {ext }}=\boldsymbol{H}_{0}=H_{0} \boldsymbol{e}_{x}$ of variable strength is applied transverse to the rotation axis $\boldsymbol{e}_{z}$ of the cylinder. $H_{0}$ is measured by a Hall detector.

In order to guarantee solid-body rotation of the fluid the container is partitioned by an array of densely packed plastic straws (inset of Fig. 8) of inner diameter $\simeq 2 \mathrm{~mm}$, which compares to the viscous skin depth.

To measure frequency and damping of the pendulum we performed a computer controlled resonance experiment: the frequency band $32 \mathrm{~Hz}<f<35 \mathrm{~Hz}$ is scanned and the resulting deflection amplitude as well as the phase lag $\phi$ relative to the drive are recorded (Fig. 9). Each data point shown in Fig. 9 is the 


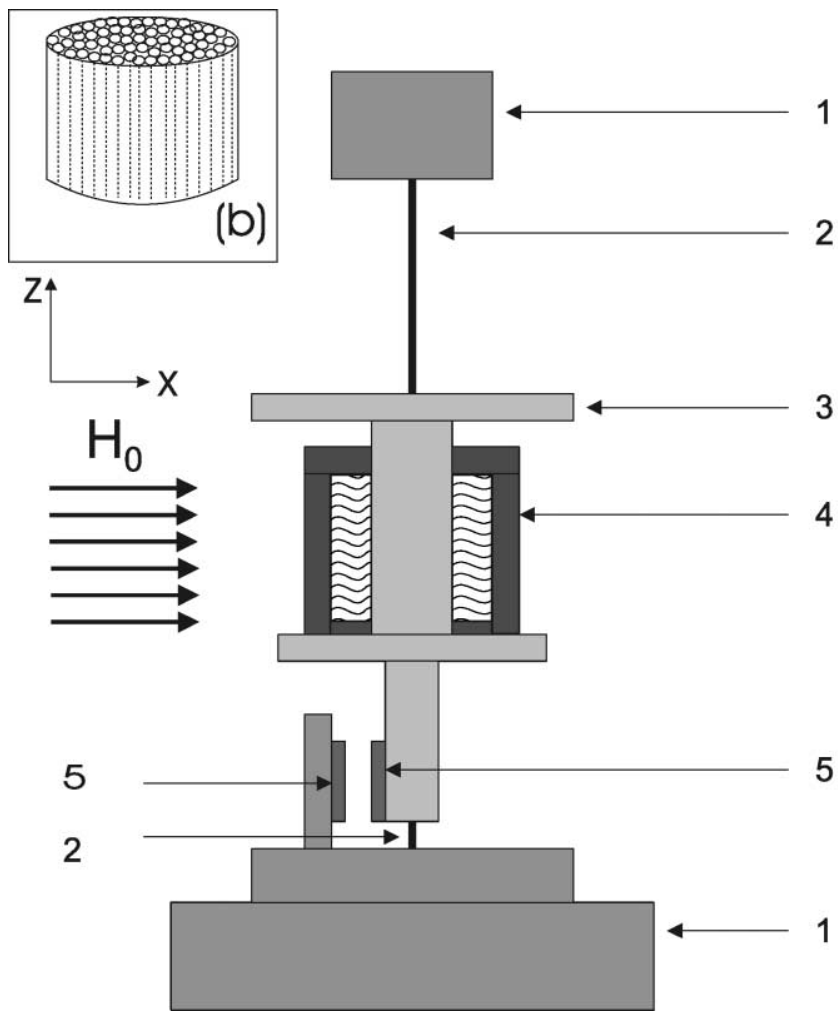

(a)

2

Fig. 8. (a) Sketch of the experimental setup. 1: outer aluminum frame; 2: torsion wire; 3: housing with carrier platform; 4: closed cylindrical Plexiglas container filled with ferrofluid; 5: capacitor for the electrostatic drive. A second capacitor (not visible here) records the deflection amplitude. The inset (b) shows the cylindrical container filled with plastic straws.

average over 50 single measurements giving a standard deviation smaller than the point size. To determine damping $\Gamma$ and eigenfrequency $\Omega$ we performed separate fits to amplitude as well as phase lag of the harmonically driven oscillator $\ddot{\varphi}+\Gamma \dot{\varphi}+\Omega^{2} \varphi=F \cos \omega t$. The agreement of the results from the two different fit-procedures was always better than $1 \%$. Performing the above described frequency scans for fields between 0 and $H_{0}=2 \times 10^{5} \mathrm{~A} / \mathrm{m}$ we obtain $\Gamma, \Omega$ as functions of $H_{0}$.

\subsubsection{Theoretical considerations}

The solid-body rotation, $\dot{\varphi} \boldsymbol{e}_{z}$, of the ferrofluid creates a nonequilibrium situation where the magnetization is forced out of the direction of the magnetic 


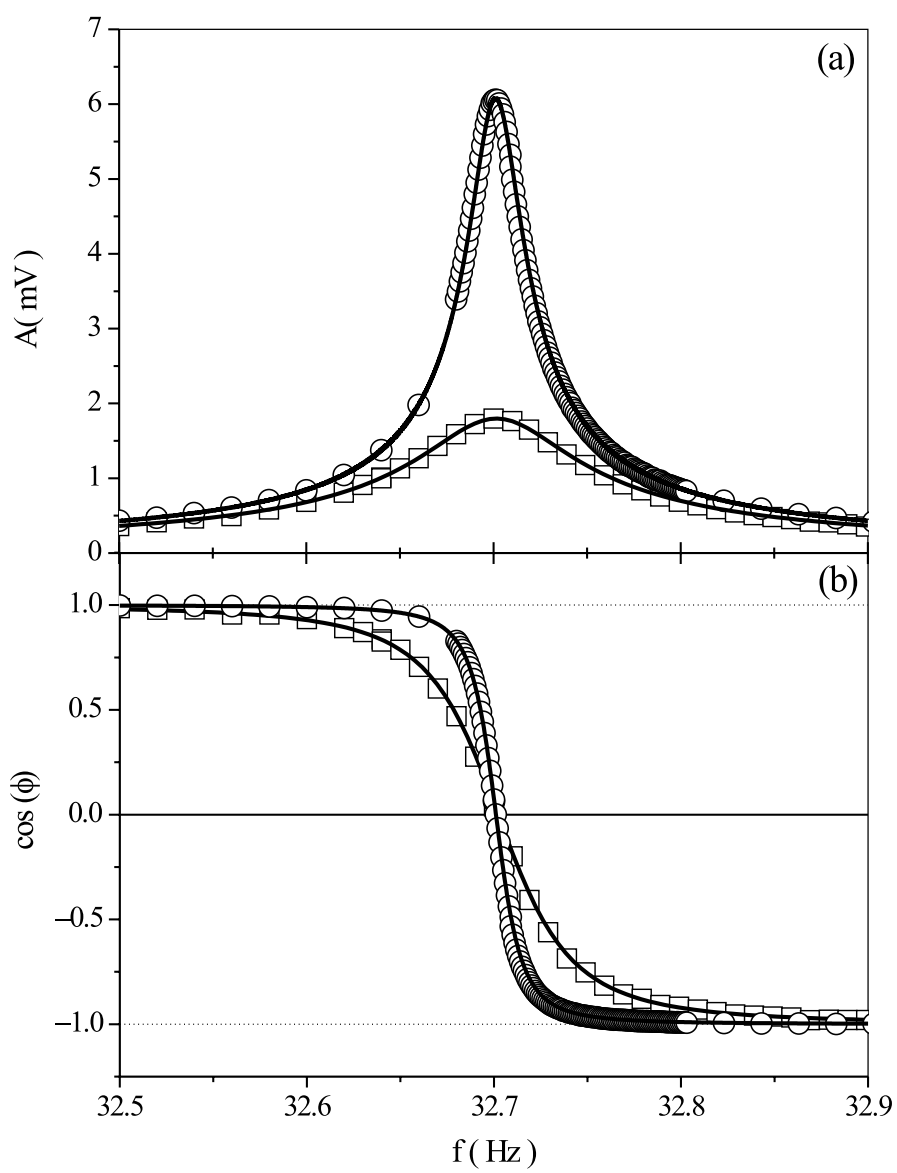

Fig. 9. Experimental raw data for the deflection amplitude (a) (measured in $\mathrm{mV}$ at the capacitor detector) and for the phase lag (b) relative to the external drive signal. Circles denote data taken for $H_{0}=0$, squares are for $H_{0}=14.2 \times 10^{4} \mathrm{~A} / \mathrm{m}$. Solid lines are fits to the harmonically driven linear oscillator. For the damping coefficient we obtain $\Gamma=0.521 \mathrm{~Hz}\left(\Gamma_{\mathrm{P}}=0.178 \mathrm{~Hz}\right)$ in the presence (absence) of the magnetic field.

field. This generates the torque [31]

$$
N=\int_{V} \mu_{0}(\boldsymbol{M} \times \boldsymbol{H})_{z} \mathrm{~d} V
$$

acting in $z$-direction on the ferrofluid volume $V$. Here $\boldsymbol{H}$ denotes the macroscopic magnetic field inside the ferrofluid probe. The magnetization $\boldsymbol{M}(\boldsymbol{r}, t)$ is an average over a large number of ferromagnetic particles, each carrying a magnetic moment $\boldsymbol{m}$, say. Their statistics is largely governed by the Langevin 
parameter $\alpha_{\text {local }}=\boldsymbol{m} \boldsymbol{H}_{\text {local }} /\left(k_{\mathrm{B}} T\right)$, where $k_{\mathrm{B}}$ is the Boltzmann constant and $T$ the absolute temperature. The local field $\boldsymbol{H}_{\text {local }}=\boldsymbol{H}_{0}-\bar{N} \boldsymbol{M}+\frac{1}{3} \boldsymbol{M}$ seen by an individual dipole differs from the local macroscopic field $\boldsymbol{H}=\boldsymbol{H}_{0}-\bar{N} \boldsymbol{M}$ by the Lorentz field of the cavity which encloses the particle [20,21]. The demagnetizing field $\bar{N} \boldsymbol{M}$ is geometry dependent; for our cylindrical container of aspect ratio $\simeq 1$ we get [38] $\bar{N} \simeq 0.3485$ so that $\boldsymbol{H}_{\text {local }} \simeq \boldsymbol{H}_{0}$ and $\alpha_{\text {local }} \simeq \alpha_{0}$. Furthermore, the tiny oscillation amplitude of our apparatus guarantees that $\boldsymbol{M}$ deviates only slightly from the equilibrium magnetization $\boldsymbol{M}_{\mathrm{eq}}=M_{\mathrm{eq}} \boldsymbol{H}_{0} / H_{0}$ in the absence of rotation, $\dot{\varphi}=0$.

We model the dynamics of the nonequilibrium magnetization $\boldsymbol{M}-\boldsymbol{M}_{\mathrm{eq}}$ by the relaxation approximation

$$
\left[\partial_{t}+(\boldsymbol{v} \nabla)-\frac{\nabla \times \boldsymbol{v}}{2} \times\right] \boldsymbol{M}=-\frac{1}{\tau_{\|}}\left(\boldsymbol{M}_{\|}-\boldsymbol{M}_{\mathrm{eq}}\right)-\frac{1}{\tau_{\perp}} \boldsymbol{M}_{\perp},
$$

which can also be motivated by studying single-particle rotary Brownian motion [39]. Here $\boldsymbol{v}(\boldsymbol{r}, t)=\dot{\varphi}\left(\boldsymbol{e}_{z} \times \boldsymbol{r}\right)$ is the velocity field and $\|$ and $\perp$ denote the respective directions parallel and perpendicular to $\boldsymbol{H}_{0}$. The quantities $\tau_{\|}, \tau_{\perp}$ are the associated field dependent relaxation times. In the limit $H_{0}=0$ they reduce to the Brownian rotational diffusion time, $\tau_{\mathrm{B}}$. Typically $\tau_{\mathrm{B}}$ lies between $10^{-3}-$ $10^{-6} \mathrm{~s}$ depending on the shape of the particle and the viscosity of the carrier liquid. With increasing field strength the relaxation times decrease according to complicated analytical expressions [39]. Ignoring spatial inhomogeneities and higher order contributions $\propto \dot{\varphi}\left(M_{\|}-M_{\text {eq }}\right)$ in Eq. (10) the magnetization $M_{y}$ in the relevant direction perpendicular to $\boldsymbol{H}_{0}$ and the rotation axis is given by

$$
\partial_{t} M_{y}=\dot{\varphi} M_{\mathrm{eq}}-\frac{1}{\tau_{\perp}} M_{y} .
$$

Thus, in the low-frequency limit, $\tau_{\perp} \Omega_{0} \ll 1$, which prevails in our experiment

$$
M_{y}(t)=\tau_{\perp} \dot{\varphi}(t) M_{\mathrm{eq}}
$$

follows the rotation frequency $\dot{\varphi}(t)$ without phase lag [40]. In this limit the torque (9) is purely dissipative

$$
N \simeq N_{\mathrm{d}}=-V \mu_{0} H_{0} M_{\mathrm{eq}} \tau_{\perp} \dot{\varphi},
$$

whereas in general the magneto-viscous torque resulting from (11) also acquires a reactive contribution. It gives rise to an $O\left(\Omega_{0} \tau_{\perp} \Gamma_{\mathrm{FF}}\right)$-increase in the eigenfrequency of the pendulum which, however, cannot be resolved with our present apparatus.

The overall damping rate $\Gamma\left(H_{0}\right)=\Gamma_{\mathrm{P}}+\Gamma_{\mathrm{FF}}\left(H_{0}\right)$ measured with our pendulum (see Fig. 8) is due to parasitic effects like air friction and mechanical 


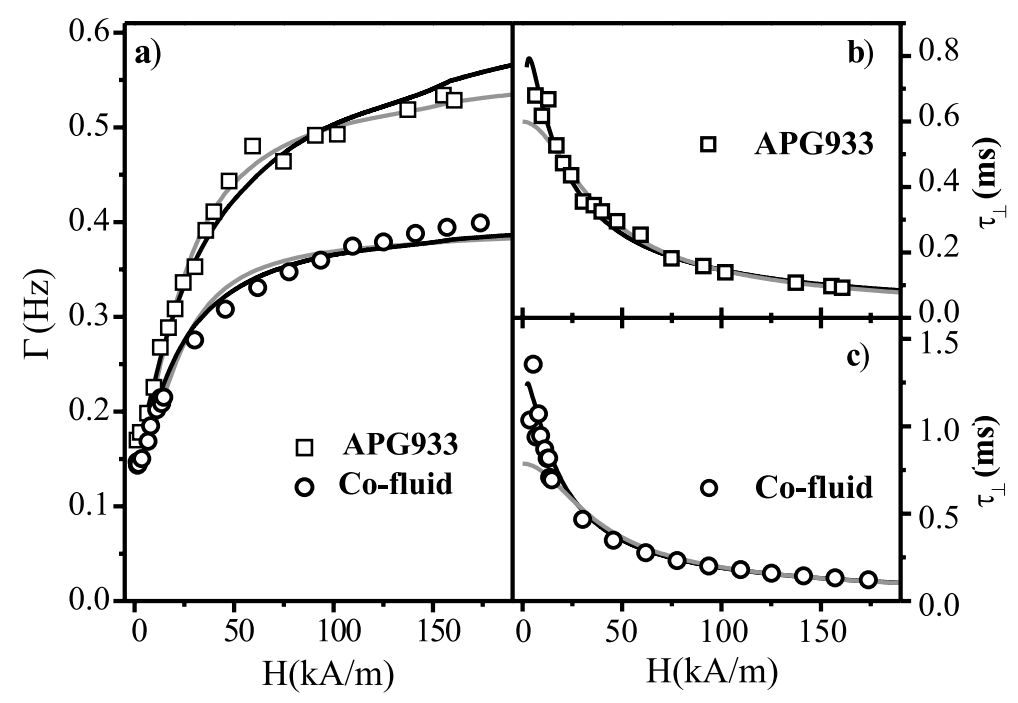

Fig. 10. The symbols denote the damping coefficient $\Gamma=\Gamma_{\mathrm{FF}}+\Gamma_{\mathrm{P}}$ measured as a function of the external magnetic field $H_{0}$. The solid line is a fit according to Eq. (15).

losses with $\Gamma_{\mathrm{P}} \simeq \Gamma\left(H_{0}=0\right)$ and to the magneto-viscous dissipation in the ferrofluid

$$
\Gamma_{\mathrm{FF}}=\frac{-N_{\mathrm{d}}}{\dot{\varphi} \Theta} .
$$

The latter is given by the total moment of inertia $\Theta$ and the oscillation frequency $\dot{\varphi}$ of the pendulum and the dissipative torque $N_{\mathrm{d}}$ of the ferrofluid. With the theoretical prediction (13) one has

$$
\Gamma_{\mathrm{FF}}=\frac{V \mu_{0} H_{0} M_{\mathrm{eq}} \tau_{\perp}}{\Theta}=\frac{V \mu_{0} M_{\mathrm{s}} k_{\mathrm{B}} T}{m \Theta} \alpha_{0} \mathcal{L}_{0} \tau_{\perp} .
$$

The second equality holds for monodisperse particles with $M_{\mathrm{eq}}=M_{\mathrm{s}} \mathcal{L}_{0}$ where $M_{\mathrm{s}}$ denotes saturation and $\mathcal{L}_{0}=\operatorname{coth} \alpha_{0}-1 / \alpha_{0}$ is the Langevin function. Note that $\Gamma_{\mathrm{FF}}$ is expressed as a function of the external magnetic field strength $\alpha_{0}$. The solid line in Fig. 10 is a fit according to Eq. (15) with $\tau_{\perp}\left(\alpha_{0}\right)$ taken from Ref. [39]. The magnetic moment $m$ of the particles, the Brownian relaxation time $\tau_{\mathrm{B}}$, and the parasitic damping $\Gamma_{\mathrm{P}}$ are considered as fit parameters in Fig. 10 leading to values $m=2.91 \times 10^{-25} \mathrm{~V} \mathrm{~s} \mathrm{~m}, \tau_{\mathrm{B}}=4.68 \times 10^{-4} \mathrm{~s}$, and $\Gamma_{\mathrm{P}}=0.178 \mathrm{~Hz}$. Assuming spherical particles of hydrodynamic diameter $d_{\mathrm{h}}$ (core plus surfactant coating) with magnetite cores of diameter $d_{\mathrm{m}}$ we get $d_{\mathrm{m}} \simeq 9.9 \mathrm{~nm}$ and $d_{\mathrm{h}} \simeq 13.5 \mathrm{~nm}$. These values are in good agreement with the manufacturer's specification [40]. For the estimate of $d_{\mathrm{h}}$ and $d_{\mathrm{m}}$ we used the relations $m=(\pi / 6) \mu_{0} M_{\mathrm{d}} d_{\mathrm{m}}^{3}$ and $\tau_{\mathrm{B}}=\pi \eta d_{\mathrm{h}}^{3} /\left(2 k_{\mathrm{B}} T\right)$, where $M_{\mathrm{d}}=4.5 \times 10^{5} \mathrm{~A} / \mathrm{m}$ 
is the bulk magnetization of magnetite and $\eta$ the shear viscosity of the ferrofluid.

\subsubsection{Rotational viscosity}

The rotational viscosity $\eta_{\mathrm{R}}\left(H_{0}\right)$ for our solid-body flow $\boldsymbol{v}=\dot{\varphi} \boldsymbol{e}_{z} \times \boldsymbol{r}$ can be identified by equating the magneto-viscous body force $\frac{\mu_{0}}{2} \nabla \times(\boldsymbol{M} \times \boldsymbol{H})$ to $\eta_{\mathrm{R}} \nabla^{2} \boldsymbol{v}$ [31]. One thus finds that

$$
\eta_{\mathrm{R}}=\frac{1}{4} \frac{-N_{\mathrm{d}} / V}{\dot{\varphi}}=\frac{\Theta}{4 V} \Gamma_{\mathrm{FF}}
$$

is given by the ratio of dissipative torque density $N_{\mathrm{d}} / V$ and rotation rate $\dot{\varphi}$. Thus, the last equality in (16) provides a simple prescription for measuring $\eta_{\mathrm{R}}\left(H_{0}\right)$ with our apparatus. It is the core result for using the torsional pendulum as a device to determine the field dependent rotational viscosity of a ferrofluid.

If one inserts Eq. (15) one gets (see also [41])

$$
\eta_{\mathrm{R}}=\frac{1}{4} \mu_{0} H_{0} M_{\mathrm{eq}} \tau_{\perp} \simeq \frac{3}{2} \eta \Phi_{\mathrm{h}} \frac{\alpha_{0} \mathcal{L}_{0}^{2}}{\alpha_{0}-\mathscr{L}_{0}} .
$$

In the last expression we have replaced $\tau_{\perp}$ by the approximation $[39,42]$ $\tau_{\perp}=2 \tau_{\mathrm{B}} \mathcal{L}_{0} /\left(\alpha_{0}-\mathscr{L}_{0}\right)$ that deviates by less than $4 \%$ from the exact $\tau_{\perp}$. Here $\Phi_{\mathrm{h}}=\Phi_{\mathrm{m}}\left(d_{\mathrm{h}} / d_{\mathrm{m}}\right)^{3}$ is the hydrodynamic volume fraction. The $\alpha_{0}$-dependence of Eq. (17) deviates by $16 \%$ from the relation derived by Shliomis [31], who found $\left(\alpha_{0}-\tanh \alpha_{0}\right) /\left(\alpha_{0}+\tanh \alpha_{0}\right)$ instead. This difference comes from his $\tau_{\perp}^{S}[40]$ being different from $\tau_{\perp}[39,42]$.

\subsubsection{Discussion}

Thus, the theory of Shliomis provides a reasonable prediction for $\eta_{\mathrm{R}}$ for our ferrofluid with a magnetic volume fraction of $\Phi_{\mathrm{m}}=3.3 \%$. This has to be seen on the background of partly conflicting previous results that we shortly discuss below. The rotational viscosity deduced by McTague from his through-flow experiment agreed qualitatively with the single-particle theory despite the fact that his $\Phi_{\mathrm{m}}$ was so high that one would expect particle correlations to show up. Weser and Stierstadt $[21,34]$ investigated a ferrofluid with $\Phi_{\mathrm{m}} \simeq 6.5 \%$ by means of a viscobalance. On taking into account the polydispersity and also parasitic viscosity contributions from excess surfactant molecules they were able to confirm Shliomis' theory by fitting the effective depth of the steric particle coating to be $25 \%$ smaller than the length of the surfactant molecules. Subsequently $\eta_{\mathrm{R}}$ was determined from the onset of Taylor vortex flow in a Couette viscometer. Here, Holderied et al. [35] reported satisfactory agreement with the theory for a ferrofluid with $\Phi_{\mathrm{m}} \simeq 7.1 \%$, while Ambacher et al. [36] and Odenbach et al. [37] observed for a less concentrated ferrofluid with $\Phi_{\mathrm{m}}=6.7 \%$ considerable discrepancies. They argued that the Shliomis approach applies only to very diluted ferrofluids with $\Phi_{\mathrm{m}}<1 \%$ and that the 
detected deviations are due to particle interactions (e.g. chain formation) not covered by the theory.

For a quantitative comparison between theory and experiment the above listed experiments have to cope with (some of) the following problems: (i) How to ensure that the assumed flow profiles (Poiseuille pipe flow, circular Couette flow, etc.) remain undeformed when the magnetic field is applied? (ii) The reported flows exhibit shear as well as rotational friction giving rise to a total effective viscosity, from which the small rotational contribution has to be separated. (iii) The important problem of identifying the proper magnetic field entering into the theoretical analysis of the experiments ( $c f$. our discussion of local vs. macroscopic field) has largely been ignored - Refs. [21,34] are an exception. (iv) For magnetizations $\boldsymbol{M}$ that deviate strongly from (local) equilibrium $\boldsymbol{M}_{\text {eq }}$ a reliable constitutive equation as required in the analysis is not available.

With the low-frequency, small-angle, rigid-body rotation of the fluid in our torsional viscometer we avoid or solve these problems and we obtain a direct and sensitive measurement of the rotational viscosity in a novel and straightforward way. By an appropriate choice of the probe geometry it was possible to evaluate the dissipative torque in terms of the (easily accessible) external magnetic field strength. For the moderately concentrated ferrofluid investigated here the results compare reasonably well with theoretical predictions based upon single-particle relaxation behavior and monodisperse size distribution.

\subsection{Effect of polydispersity}

Polydispersity strongly influences the macroscopic magnetic properties of the ferrofluid. We investigate here itseffect on the dynamic response of a ferrofluid pendulum [43].

A torsional pendulum containing a ferrofluid is forced periodically in a homogeneous magnetic field $\boldsymbol{H}^{\mathrm{ext}}=H^{\mathrm{ext}} \boldsymbol{e}_{x}$ that is applied perpendicular to the pendulum axis $\boldsymbol{e}_{z}$ (see Fig. 11). Such a ferrofluid pendulum is used for measuring the rotational viscosity $[16,32]$. The cylindrical ferrofluid container is here of sufficiently large length to be approximated as an infinite long cylinder. We consider rigid-body rotation of the ferrofluid with the time dependent angular velocity $\boldsymbol{\Omega}=\dot{\varphi} \boldsymbol{e}_{z}$ as can be realized with the set-up of $[16,32]$. The fields $\boldsymbol{H}$ and $\boldsymbol{M}$ inside the cylinder are spatially homogeneous and oscillating in time.

\subsubsection{Equations}

First, the Maxwell equations demand that the fields $\boldsymbol{H}$ and $\boldsymbol{M}$ within the ferrofluid are related to each other via

$$
\boldsymbol{H}+\bar{N} \boldsymbol{M}=\boldsymbol{H}^{\mathrm{ext}},
$$




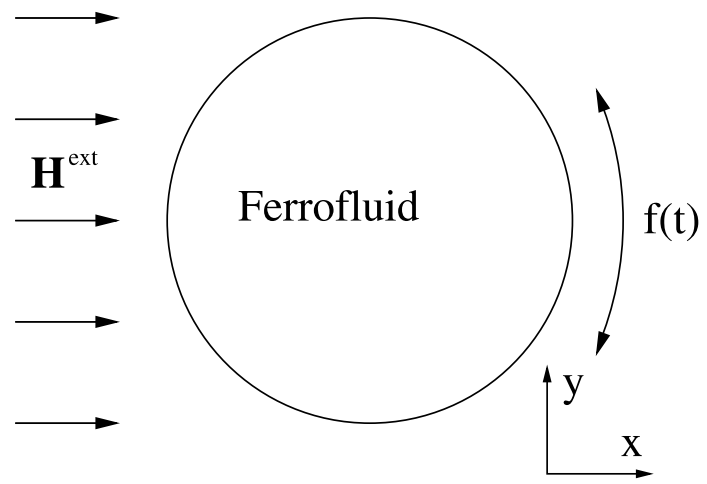

Fig. 11. Schematic plot of the FF-pendulum.

with $\bar{N}=1 / 2$ for the infinitely long cylinder. Then we have the torque balance of the torsional pendulum

$$
\ddot{\varphi}=-\omega_{0}^{2} \varphi-\Gamma_{0} \dot{\varphi}+\frac{N}{\Theta}+f(t),
$$

with the eigenfrequency $\omega_{0}$ and the damping rate $\Gamma_{0}$ of the pendulum without field and the total moment of inertia $\Theta$. The magnetic field $\boldsymbol{H}$ exerts on the magnetic moments of the ferrofluid, i.e., on the pendulum the torque

$$
N=\mu_{0} \int d V(\boldsymbol{M} \times \boldsymbol{H})_{z}=-\mu_{0} V M_{y} H^{\mathrm{ext}},
$$

and $f(t)$ is the external mechanical forcing.

Finally, we need an equation describing the magnetization dynamics. Here, we consider the polydisperse ferrofluid as a mixture of ideal monodisperse paramagnetic fluids. Then the resulting magnetization is given by $\boldsymbol{M}=\sum \boldsymbol{M}_{j}$, where $\boldsymbol{M}_{j}$ denotes the magnetization of the particles with diameter $d_{j}$. We assume that each $\boldsymbol{M}_{j}$ obeys a simple Debye relaxation dynamics described by

$$
d_{t} \boldsymbol{M}_{j}-\boldsymbol{\Omega} \times \boldsymbol{M}_{j}=-\frac{1}{\tau_{j}}\left[\boldsymbol{M}_{j}-\boldsymbol{M}_{j}^{\mathrm{eq}}(\boldsymbol{H})\right] .
$$

We take the equilibrium magnetization to be given by a Langevin function

$$
\boldsymbol{M}_{j}^{\mathrm{eq}}(\boldsymbol{H})=\chi_{j}(H) \boldsymbol{H}=w_{j} \mathcal{L}\left(\frac{\mu_{0} \pi M_{\mathrm{mat}}}{6 k_{\mathrm{B}} T} d_{j}^{3} H\right) \frac{\boldsymbol{H}}{H},
$$

with the saturation magnetization of the material $M_{\text {mat }}$ and the magnetization distribution $w_{j}\left(d_{j}\right)$. Note that the magnetization equations (21) for the different particle sizes are coupled by the internal field $\boldsymbol{H}=\boldsymbol{H}^{\mathrm{ext}}-\bar{N} \boldsymbol{M}$. As relaxation rate we combine Brownian and Néel relaxation $1 / \tau_{j}=1 / \tau_{\mathrm{B}}^{j}+1 / \tau_{\mathrm{N}}^{j}$. The 
relaxation times depend on the particle size by $\tau_{\mathrm{B}}^{j}=\pi \eta / 2 k_{\mathrm{B}} T\left(d_{j}+2 s\right)^{3}$ and $\tau_{\mathrm{N}}^{j}=f_{0}^{-1} \exp \left(\pi K d_{j}^{3} / 6 k_{\mathrm{B}} T\right)$ with $\eta$ the viscosity, $s$ the thickness of the nonmagnetic particle layer and $K$ the anisotropy constant.

Altogether we use the following system of equations:

$$
\begin{aligned}
\dot{\varphi} & =\Omega \\
\dot{\Omega} & =-\omega_{0}^{2} \varphi-\Gamma_{0} \Omega-\mu_{0} \frac{V}{\Theta} H^{\mathrm{ext}} M_{y}+f(t) \\
\dot{M}_{x}^{j} & =-\Omega M_{y}^{j}-\frac{1}{\tau_{j}}\left[M_{x}^{j}-\chi_{j}(H)\left(H^{\mathrm{ext}}-\bar{N} M_{x}\right)\right] \\
\dot{M}_{y}^{j} & =\Omega M_{x}^{j}-\frac{1}{\tau_{j}} M_{y}^{j}-\frac{1}{\tau_{j}} \bar{N} \chi_{j}(H) M_{y} .
\end{aligned}
$$

\subsubsection{Linear response analysis}

First we should like to emphasize that we use in this paragraph the index 0 to identify the equilibrium situation of the unforced pendulum at rest: $\varphi_{0}=\Omega_{0}=M_{y}^{j 0}=0$ and $M_{x}^{j 0}=M_{\mathrm{eq}}^{j}\left(H_{0}\right)$. Furthermore, $M_{0}=\sum M_{\mathrm{eq}}^{j}\left(H_{0}\right)$ with $H_{0}$ being the internal field in the pendulum at rest, i.e., $H_{0}=H^{\mathrm{ext}}-\bar{N} M_{0}\left(H_{0}\right)$.

External forcing with small $|f|$ leads to small deviations of $\varphi$, of $\Omega$, and of $\delta \boldsymbol{H}=\boldsymbol{H}-\boldsymbol{H}_{0}=-\bar{N}\left(\boldsymbol{M}-\boldsymbol{M}_{0}\right)=-\bar{N} \delta \boldsymbol{M}$ from the above described equilibrium state. We expand each $\chi_{j}(H)$ up to linear order in $\delta \boldsymbol{H}$

$$
\chi_{j}\left(\left|\boldsymbol{H}_{0}+\delta \boldsymbol{H}\right|\right)=\chi_{j 0}-\chi_{j 0}^{\prime} \bar{N} \delta M_{x}+\mathcal{O}(\delta \boldsymbol{H})^{2} .
$$

Here, $\chi_{j 0}=\chi_{j}\left(H_{0}\right)$ and $\chi_{j 0}^{\prime}$ is the derivative of $\chi_{j 0}$. Then we get the linearized equations

$$
\begin{aligned}
\dot{\varphi} & =\Omega \\
\dot{\Omega} & =-\omega_{0}^{2} \varphi-\Gamma_{0} \Omega-\kappa y+f(t) \\
\dot{x}_{j} & =-\frac{1}{\tau_{j}} x_{j}-\frac{1}{\tau_{j}} \bar{N}\left(\chi_{j 0}+\chi_{j 0}^{\prime} H_{0}\right) x \\
\dot{y}_{j} & =\Omega x_{j}^{0}-\frac{1}{\tau_{j}} y_{j}-\frac{1}{\tau_{j}} \bar{N} \chi_{j 0} y .
\end{aligned}
$$

We have introduced the abbreviations $x_{j}=\delta M_{x} / M_{0}, x_{j}^{0}=M_{x}^{j, 0} / M_{0}, y_{j}=$ $\delta M_{y} / M_{0}$ and $x=\sum_{j} x_{j}, y=\sum_{j} y_{j}$. The strength of the coupling constant between the mechanical degrees of freedom $\varphi, \Omega$ and the magnetic ones is $\kappa=\mu_{0} H^{\mathrm{ext}} M_{0} V / \Theta$.

For periodic forcing $f(t)=\hat{f}^{-\mathrm{i} \omega t}$ we look for solutions in the form

$$
\left(\begin{array}{c}
\varphi(t) \\
\Omega(t) \\
x_{j}(t) \\
y_{j}(t)
\end{array}\right)=\left(\begin{array}{c}
\hat{\varphi} \\
\hat{\Omega} \\
\hat{x}_{j} \\
\hat{y}_{j}
\end{array}\right) \mathrm{e}^{-\mathrm{i} \omega t} .
$$


Inserting the ansatz (32) into the linearized equations (28)-(31) yields

$$
\begin{aligned}
\hat{\Omega} & =-\mathrm{i} \omega \hat{\varphi} \\
\hat{x} & =0=\hat{x}_{j} \\
\hat{y}_{j} & =-\left[\frac{\mathrm{i} \omega \tau_{j}}{1-\mathrm{i} \omega \tau_{j}} x_{j}^{0}-\frac{\bar{N} \chi_{j 0}}{1-\mathrm{i} \omega \tau_{j}} \frac{\omega}{\kappa} \Sigma\right] \hat{\varphi} \\
\hat{y} & =-\frac{\omega}{\kappa} \Sigma \hat{\varphi}
\end{aligned}
$$

and

$$
\hat{\varphi}=G \hat{f}=\left[\omega_{0}^{2}-\omega^{2}-\mathrm{i} \omega \Gamma_{0}-\omega \Sigma\right]^{-1} \hat{f} .
$$

The ferrofluid-induced 'selfenergy' $\Sigma(\omega)$ in the expression for the dynamical response function $G(\omega)$ of the torsional pendulum is

$$
\Sigma(\omega)=\mathrm{i} \kappa\left(1+\bar{N} \sum_{j} \frac{\chi_{j 0}}{1-\mathrm{i} \omega \tau_{j}}\right)^{-1} \sum_{j} \frac{\tau_{j} x_{j}^{0}}{1-\mathrm{i} \omega \tau_{j}} .
$$

Its imaginary part changes the damping rate $\Gamma_{0}$ of the pendulum for $\kappa=0$, i.e., in zero field. The real part shifts the resonance frequency of the pendulum. In the special case of a monodisperse ferrofluid one has

$$
\Sigma(\omega)=\frac{\mathrm{i} \kappa \tau}{1-\mathrm{i} \omega \tau+\bar{N} \chi_{0}} .
$$

\subsubsection{Results}

We evaluated the linear response function $G(\omega)=\hat{\varphi}(\omega) / \hat{f}$ of the pendulum's angular deviation amplitude $\hat{\varphi}(\omega)$ to the applied forcing amplitude $\hat{f}$ and the selfenergy $\Sigma(\omega)$ for some experimental parameters from [16,32]: $\omega_{0} / 2 \pi=32.7 \mathrm{~Hz}, \Gamma_{0}=0.178 \mathrm{~Hz}, V / \Theta=20 \mathrm{~m} / \mathrm{kg}$. The cylinder is filled with the ferrofluid APG 933 of FERROTEC. Therefore, we used in Eq. (39) an experimental $\tau=0.6 \mathrm{~ms}$ and the experimental $M_{\mathrm{eq}}(H)$. These monodisperse results were compared with the expression (38) for the polydisperse case for the typical parameter values $M_{\text {mat }}=456 \mathrm{kA} / \mathrm{m}, \eta=0.5 \mathrm{Pas}, s=2 \mathrm{~nm}$, $K=44 \mathrm{~kJ} / \mathrm{m}^{3}$ and $f_{0}=10^{9} \mathrm{~Hz}$. The contributions $w\left(d_{j}\right)$ that enter into the formulas (22) for the susceptibilities $\chi_{j}$ are given by a lognormal distribution [17]:

$$
\begin{aligned}
& w\left(d_{j}\right)=M_{\text {sat }} \frac{g\left(d_{j}\right) d_{j}}{\sum_{k=1}^{30} g\left(d_{k}\right) d_{k}} \\
& \text { with } g\left(d_{j}\right)=\frac{1}{\sqrt{2 \pi} d_{j} \ln \sigma} \exp \left(-\frac{\ln ^{2}\left(d_{j} / d_{0}\right)}{2 \ln ^{2} \sigma}\right) .
\end{aligned}
$$




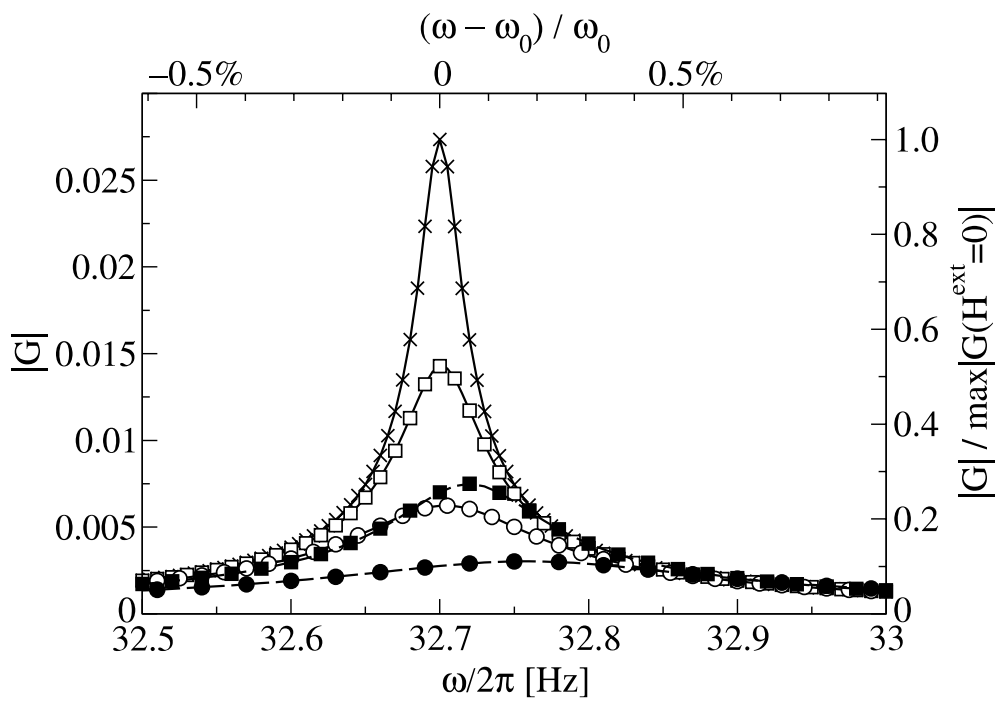

Fig. 12. $|G|$ near the resonance $\omega_{0} ; \times H^{\text {ext }}=0 \mathrm{kA} / \mathrm{m}$, squares $H^{\text {ext }}=5 \mathrm{kA} / \mathrm{m}$, circles $H^{\text {ext }}=10 \mathrm{kA} / \mathrm{m}$; filled symbols: polydisperse.

Fitting the experimental $M_{\mathrm{eq}}(H)$ with a sum of Langevin functions (22) yields $M_{\text {sat }}=18149 \mathrm{~A} / \mathrm{m}, d_{0}=7 \mathrm{~nm}$ and $\sigma=1.47$. We used here 30 different particle sizes from $d_{1}=1 \mathrm{~nm}$ to $d_{30}=30 \mathrm{~nm}$.

The calculations show the additional damping rate caused by the interaction between ferrofluid and external field. An increasing magnetic field leads to smaller amplitudes; in polydisperse ferrofluids the amplitude decreases faster (Figs. 12 and 13a). Furthermore, one can see a shift of the peak position to higher frequencies $\omega_{\max }$, which is stronger in polydisperse ferrofluids (Figs. 12, $13 \mathrm{~b}$ and $14 \mathrm{a})$.

\section{Rotating ferrofluid cylinder in a magnetic field}

The prospect of influencing the rotational dynamics of the nanoscaled magnetic particles in a ferrofluid by a macroscopic flow and/or by a magnetic field in order to then observe the resulting response via the magnetization and/or via changes in the flow has been stimulating many research activities [15,44-46] ever since McTague measured [29] the so-called magneto-viscous effect. Of particular interest are in this context flows that are shear free on the macroscopic scale as in a fluid that is rotating like a rigid body with a rotation frequency, say, $\boldsymbol{\Omega}=\boldsymbol{\Omega} \boldsymbol{e}_{z}$.

While the colloidal magnetic particles then undergo thermally sustained rotational and translational Brownian motion on the microscopic scale they 


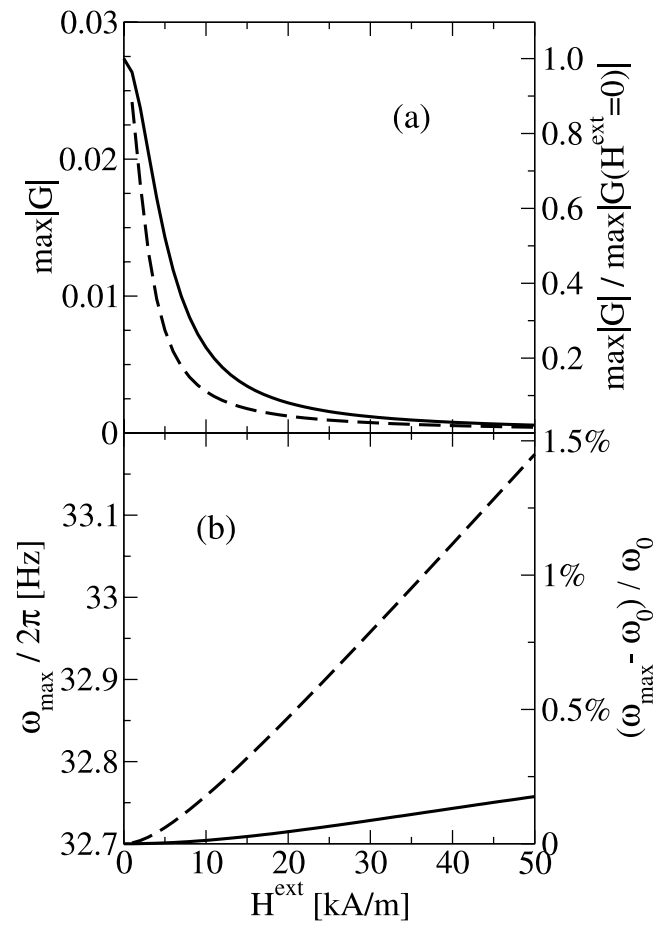

Fig. 13. Maximum value $\max |G|$ (a) and peak position $\omega_{\max }$ (b) as a function of external field $H^{\text {ext }}$; full line monodisperse, dashed line polydisperse.

co-rotate in the mean with the deterministic macroscopic rigid body flow. However, this mean co-rotation can be hindered by magnetic torques on their moments when a magnetic field, say, $\boldsymbol{H}^{\mathrm{ext}}=\boldsymbol{H}_{0}=H_{0} \boldsymbol{e}_{x}$ is applied perpendicular to the rotation axis $\boldsymbol{e}_{z}$ of the flow. The combination of the externally imposed forcing of the particle motion by (i) the rigid body flow in which they are floating and by (ii) the magnetic torques on their magnetic moments drives the colloidal suspension out of equilibrium. Concerning the magnetic moments, this forcing causes the mean orientation of the moments, i.e., of the magnetization $\boldsymbol{M}$ of the ferrofluid to be no longer parallel to the internal magnetic field $\boldsymbol{H}$. Instead, $\boldsymbol{M}$ is pushed out of the direction of $\boldsymbol{H}$ as well as of that of $\boldsymbol{H}_{0}$ thereby acquiring a nonzero transverse component $M_{y}$.

This transverse magnetization $M_{y}$ of a slender cylindrical column of ferrofluid that was enforced to rotate like a rigid body with constant frequency $\Omega \boldsymbol{e}_{z}$ in an applied homogeneous magnetic field $H_{0} \boldsymbol{e}_{x}$ was measured as a function of $\Omega$ and $H_{0}$ [47]. These measurements are compared with the predictions based on both single-relaxation time models [31,48-52] and polydisperse models with multi-relaxation times [53] for the magnetization dynamics. 


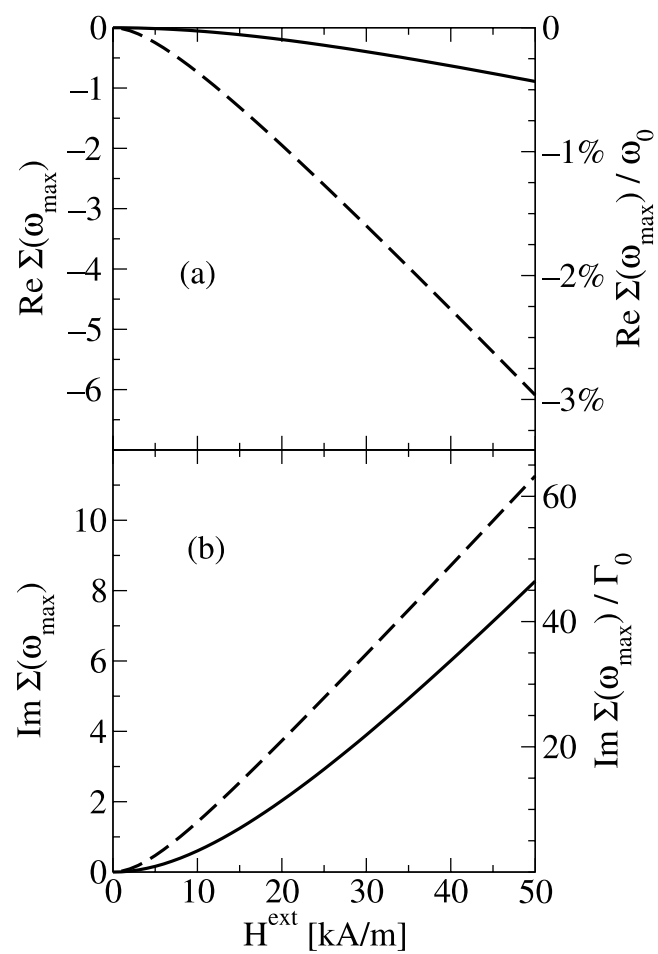

Fig. 14. $\operatorname{Re}(\Sigma)$ (a) and $\operatorname{Im}(\Sigma)$ (b) at $\omega=\omega_{\max }$; full line monodisperse, dashed line polydisperse.

\subsection{Experimental system}

The experimental setup for measuring the magnetization of a rotating cylindrical column of ferrofluid [47] is sketched in Fig. 15. The ferrofluid is filled into a cylindrical plexiglass sample holder with inside radius $R=3.2 \mathrm{~mm}$. This radius is so small that for our rotation frequencies the ferrofluid rotates as a rigid body with a flow field $\boldsymbol{u}(\boldsymbol{r})=\boldsymbol{\Omega} \times \boldsymbol{r}=\boldsymbol{\Omega} \boldsymbol{r} \boldsymbol{e}_{\varphi}$. Here $\Omega$ is the externally enforced constant rotation rate of the sample and $\boldsymbol{e}_{\varphi}$ is the unit vector in azimuthal direction.

The sample is placed centrally between the poles of an electromagnet (BRUKER B-E 10V) providing the homogeneous and temporally constant magnetic field $\boldsymbol{H}_{0}=H_{0} \boldsymbol{e}_{x}$ perpendicular to the cylinder axis $\boldsymbol{e}_{z}$.

For such a combination of enforced rotation and applied field theoretical models allow for a spatially and temporally constant nonequilibrium magnetization $\boldsymbol{M}$ that is pushed out of the directions of $\boldsymbol{H}_{0}$ and $\boldsymbol{H}$ by the flow. However, in equilibrium, $\boldsymbol{\Omega}=0$, the three fields are indeed collinear: the equilibrium magnetization $\boldsymbol{M}_{\mathrm{eq}}(\boldsymbol{H})=M_{\mathrm{eq}}(H) \boldsymbol{H} / H$ is parallel to the internal field $\boldsymbol{H}$. 


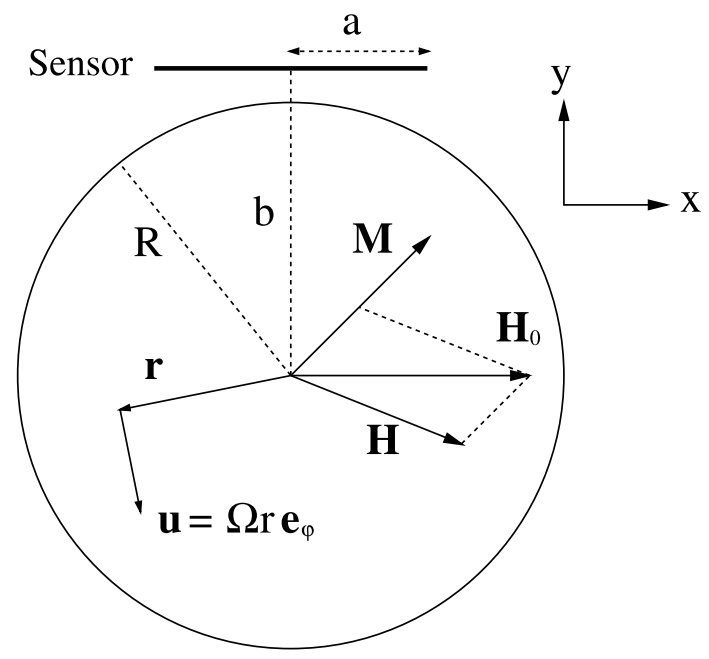

Fig. 15. Schematic plot of the rotating FF-cylinder. The cylindrical sample holder with inner radius $R$ rotates with angular velocity $\boldsymbol{\Omega}$ in the applied static magnetic field $\boldsymbol{H}_{0}$ perpendicular to $\boldsymbol{\Omega}$. The magnetization $\boldsymbol{M}_{\mathrm{y}}$ is measured with a Hall sensor. $\boldsymbol{M}$ and $\boldsymbol{H}$ denote the magnetization and internal magnetic field of the ferrofluid. Both are constant in space and time.

According to the Maxwell equations the fields $\boldsymbol{H}$ and $\boldsymbol{M}$ within the ferrofluid are related to each other via

$$
\boldsymbol{H}=\boldsymbol{H}_{0}-\frac{1}{2} \boldsymbol{M} \text {. }
$$

for our long cylindrical sample and in particular $H_{y}=-M_{y} / 2$ as indicated schematically in Fig. 15. In addition they demand that the magnetic field outside the ferrofluid cylinder

$$
\boldsymbol{H}^{\text {out }}=\boldsymbol{H}_{0}+\frac{1}{2} \frac{R^{2}}{r^{2}}\left(2 \frac{\boldsymbol{r}}{r} \frac{\boldsymbol{M r}}{r}-\boldsymbol{M}\right)
$$

is a superposition of the applied field $\boldsymbol{H}_{0}$ and the dipolar contribution from $\boldsymbol{M}$. This result yields a relation between the perpendicular component of the magnetization $M_{y}$ resp. of the internal field $H_{y}=-M_{y} / 2$ and the field $H_{y}^{\text {sensor }}$ measured by the Hall-sensor outside the sample as indicated in Fig. 15. Considering the finite size of the Hall-sensor, $H_{y}^{\text {sensor }}$ is given by

$$
H_{y}^{\text {sensor }}=\frac{1}{2 a} \int_{-a}^{a} H_{y}^{\text {out }} \mathrm{d} x=-\frac{R^{2}}{a^{2}+b^{2}} H_{y} .
$$

In our experimental setup $b=4.75 \mathrm{~mm}, R=3.2 \mathrm{~mm}$, and the horizontal extension of the Hall sensor $a=2 \mathrm{~mm}$. So, we get $H_{y}^{\text {sensor }}=-0.386 H_{y}$. 
In our experiments we used the ferrofluid APG933 of FerroTec. We have measured the equilibrium magnetization of the ferrofluids ( $c f$. Sect. 3.1.1). In order to get information on the particle size distribution of the ferrofluid under investigation, we used fits with a lognormal form of the distribution [17] as well as with a regularization procedure based on Tichonovs method [34, 47, 54].

The saturation magnetization of the ferrofluid sample was $M_{\mathrm{sat}}^{\mathrm{FF}}=$ 19108.6 A/m. From the saturation magnetization the volume concentration of the magnetite particles was found to be $\phi=M_{\mathrm{sat}}^{\mathrm{FF}} / M_{\mathrm{sat}}^{\mathrm{bulk}}=4.1 \%$. For the initial susceptibility we found the value $\chi_{0}=1.09$.

\subsection{Theoretical models}

\subsubsection{Monodisperse case}

The model equations that we compare here imply a relaxational dynamics with a single relaxation time either of $\boldsymbol{M}$ towards the equilibrium magnetization

$$
\boldsymbol{M}_{\mathrm{eq}}(\boldsymbol{H})=\frac{M_{\mathrm{eq}}(H)}{H} \boldsymbol{H}=\chi(H) \boldsymbol{H}
$$

or of the "local equilibrium" or "effective" field

$$
\boldsymbol{H}_{\mathrm{eff}}(\boldsymbol{M})=\frac{M_{\mathrm{eq}}^{-1}(M)}{M} \boldsymbol{M}=F(M) \boldsymbol{M}
$$

towards the internal field $\boldsymbol{H}$. The equilibrium magnetization $M_{\mathrm{eq}}(H)$ referring to the functional relation between internal field $H$ and magnetization in the case of $\Omega=0$ is a thermodynamic material property of the ferrofluid. The effective field $\boldsymbol{H}_{\text {eff }}$ lies parallel to $\boldsymbol{M}$ and can be seen as the inverse of the defining requirement

$$
\boldsymbol{M}=\boldsymbol{M}_{\mathrm{eq}}\left(\boldsymbol{H}_{\mathrm{eff}}\right) .
$$

In equilibrium, $\Omega=0$, one has $\boldsymbol{H}_{\mathrm{eff}}=\boldsymbol{H}$ and $\boldsymbol{M}=\boldsymbol{M}_{\mathrm{eq}}$.

We consider here the relations [48]

$$
\begin{array}{rlrl}
\text { Debye: } & \boldsymbol{\Omega} \times \boldsymbol{M} & =\frac{1}{\tau}\left(\boldsymbol{M}-\boldsymbol{M}_{\mathrm{eq}}\right) \\
\text { S'72 [31]: } & \boldsymbol{\Omega} \times \boldsymbol{M}=\frac{1}{\tau}\left(\boldsymbol{M}-\boldsymbol{M}_{\mathrm{eq}}\right)+\frac{\mu_{0}}{4 \zeta} \boldsymbol{M} \times(\boldsymbol{M} \times \boldsymbol{H}) \\
\text { FK [49, 50, 55]: } & \boldsymbol{\Omega} \times \boldsymbol{M}=\gamma_{H}\left(\boldsymbol{H}_{\mathrm{eff}}-\boldsymbol{H}\right)+\frac{\mu_{0}}{4 \zeta} \boldsymbol{M} \times(\boldsymbol{M} \times \boldsymbol{H}) \\
\text { S'01 [51, 56]: } & \boldsymbol{\Omega} \times \boldsymbol{H}_{\mathrm{eff}}=\frac{1}{\tau}\left(\boldsymbol{H}_{\mathrm{eff}}-\boldsymbol{H}\right)+\frac{\mu_{0}}{4 \zeta} \boldsymbol{H}_{\mathrm{eff}} \times(\boldsymbol{M} \times \boldsymbol{H}) \\
\text { ML [52]: } & \boldsymbol{\Omega} \times \boldsymbol{M}=\xi\left(\boldsymbol{H}_{\mathrm{eff}}-\boldsymbol{H}\right)
\end{array}
$$


resulting for the rotating cylinder from the above 5 models. Here $\tau$ denotes a magnetic relaxation time, $\gamma_{H}$ and $\xi$ relaxation rates, $\zeta$ the vortex viscosity and $\mu_{0}$ the vacuum permeability. In our calculations, we use $\gamma_{H}=\chi_{0} / \tau$ [57]. In ML we use the weak field variant of Ref. [52] with two different choices for the parameter $\xi$ : Either the $\xi=\chi_{0} / \tau$ as in FK that is denoted here by ML(F). Or the variant $\xi=1 /[F(M) \tau]$ as in S'01 that is denoted here by ML(S).

Especially the perpendicular component $H_{y}=-\frac{1}{2} M_{y}$ of the magnetic field is suited for a comparison of the different models with each other and with experiments. Before doing the former we should like to draw the attention to the frequency behavior of $M_{y}\left(H_{0}, \Omega\right)$. We mentioned already that $M_{y}$ vanishes for zero vorticity, $\Omega=0$. Furthermore, one finds that $M_{y}$ as well as $M_{x}$ vanishes also in the limit $\Omega \rightarrow \infty$. Because of $M_{y} \geq 0$ one sees, that $M_{y}(\Omega)$ has a maximum as a function of $\Omega$ [48].

The differences in the results for the different models are easily captured by comparing their predictions for the maximum values of $\left|H_{y}\right|=\frac{1}{2} M_{y}$ and the locations of these maxima at $\Omega^{\max }$, each as a function of applied field $H_{0}$.

As an aside we mention that the maximal values of $\left|H_{y}\right|$ of Debye and S'72 are the same while their locations, $\Omega^{\max }$, differ. The models S'01, FK, and ML formulated in terms of the effective field also share a common maximal value of $\left|H_{y}\right|$ being larger than that of Debye and S' 72 while the location, $\Omega^{\text {max }}$, differ partly substantially. Hence, the magnetic torque, $\boldsymbol{M} \times \boldsymbol{H}$, entering into S'72, FK, and S' 01 only shifts the frequency $\Omega^{\max }$ [48].

\subsubsection{Polydisperse case}

Here comparisons are made of the above single-relaxation-time approach with polydisperse models with multi-relaxation times [53]. We consider here as a first step polydisperse models with single-particle Brownian and Néel relaxation dynamics for the different particle sizes. Such models have been used to determine within a linear response analysis the effect of polydispersity on the dynamics of a torsional ferrofluid pendulum that was periodically forced close to resonance to undergo small amplitude oscillations in a rigid body flow $[16,32,43]$.

We ignore the influence of any dipolar magnetic interaction and of any flow induced interaction on the (rotation) dynamics of the particles. Thereby collective, collision dominated long-range and long-time hydrodynamic relaxation dynamics of the ensemble of magnetic moments are discarded since only the individual relaxation of each magnetic moment is considered - albeit in the collectively generated internal magnetic field $\boldsymbol{H}$.

For numerical reasons we use a discrete partition of the particle size distribution. Then, without interaction, the magnetization of the resulting mixture of mono-disperse ideal paramagnetic gases is given by $\boldsymbol{M}=\sum \boldsymbol{M}_{j}$, where $\boldsymbol{M}_{j}$ denotes the magnetization of the particles with diameter $d_{j}$. We assume that each magnetic moment and with it each $\boldsymbol{M}_{j}$ obeys a simple Debye relaxation dynam- 
ics that drives them in the absence of flow towards their respective equilibrium value $\boldsymbol{M}_{j}^{\mathrm{eq}}(\boldsymbol{H})$. Then, in the stationary situation resulting from the rigid body rotation with constant $\boldsymbol{\Omega}$ the Debye relaxation equation for each sub magnetization (21) reduces to

$$
\boldsymbol{\Omega} \times \boldsymbol{M}_{j}=\frac{1}{\tau_{j}}\left[\boldsymbol{M}_{j}-\boldsymbol{M}_{j}^{\mathrm{eq}}(\boldsymbol{H})\right] .
$$

In the absence of interactions the equilibrium magnetization $\boldsymbol{M}_{j}^{\mathrm{eq}}(\boldsymbol{H})$ of each species is determined by a Langevin function (22). For the magnetic weight $w_{j}\left(d_{j}\right)$ of species $j$ appearing in (22) we take the experimentally determined values from the representation $\boldsymbol{M}^{\mathrm{eq}}=\sum \boldsymbol{M}_{j}^{\mathrm{eq}}$. Note that the magnetization equations (52) for the different particle sizes are coupled via the internal field $\boldsymbol{H}$ : according to Maxwell's equations $\boldsymbol{H}=\boldsymbol{H}_{0}-\frac{1}{2} \boldsymbol{M}$ is given in terms of the total $\boldsymbol{M}=\sum \boldsymbol{M}_{j}$.

In the relaxation rate $1 / \tau_{j}$ we take into account Brownian and Néel relaxation processes by adding their rates with equal weight

$$
\frac{1}{\tau_{j}}=\frac{1}{\tau_{\mathrm{B}}^{j}}+\frac{1}{\tau_{\mathrm{N}}^{j}} .
$$

The relaxation times depend on the particle size according to $\tau_{\mathrm{B}}^{j}=\pi \eta / 2 k_{\mathrm{B}} T$ $\left(d_{j}+2 s\right)^{3}$ and $\tau_{\mathrm{N}}^{j}=f_{0}^{-1} \exp \left(\pi K d_{j}^{3} / 6 k_{\mathrm{B}} T\right)$. Her $\eta$ is the viscosity, $s$ the thickness of the nonmagnetic particle layer, $K$ the anisotropy constant and $f_{0}$ an attempt frequency of $\sim 10^{9} \mathrm{~Hz}[15,58]$. The combined relaxation rate (53) is dominated by the faster of the two processes. Thus, large particles relax in a Brownian manner with relaxation times of about some $10^{-3} \mathrm{~s}$, while small particles have the much smaller Néel relaxation times. The typical range of $K$ for magnetite-based ferrofluids is about $(10-50) \mathrm{kJ} / \mathrm{m}^{3}[59,60]$. The boundary between Néel and Brownian dominated relaxation as a function of particle size $d$ depends sensitively on the anisotropy constant $K$. This is documented in Fig. 16 for the two values $K=15 \mathrm{~kJ} / \mathrm{m}^{3}$ and $K=50 \mathrm{~kJ} / \mathrm{m}^{3}$. For these specific examples the boundaries between Néel and Brownian dominated relaxation lie at about $d \simeq 20 \mathrm{~nm}$ and $d \simeq 13 \mathrm{~nm}$, respectively.

\subsection{Results}

We measured $H_{y}$ as a function of $\Omega$ ranging from 0 up to $3000 \mathrm{rad} / \mathrm{s}$ for a fixed value of $H_{0}$. After completing an experiment we increased $H_{0}$ and repeated the experiment. In this way we got $H_{y}(\Omega)$ for seven different values of $H_{0}$. One observes that the maximum amplitude of $H_{y}$ increases with increasing $H_{0}$ and that the peak position $\Omega^{\max }$ is shifted towards higher $\Omega$-values with increasing $H_{0}$.

In a first attempt we compared the experimental data for APG 933 with numerical calculations of $H_{y}^{\text {sensor }}(\Omega)$ with the single-relaxation time models (Sect. 4.2.1). Therefore we used $\tau$ as fit parameter and in addition an ad-hoc 


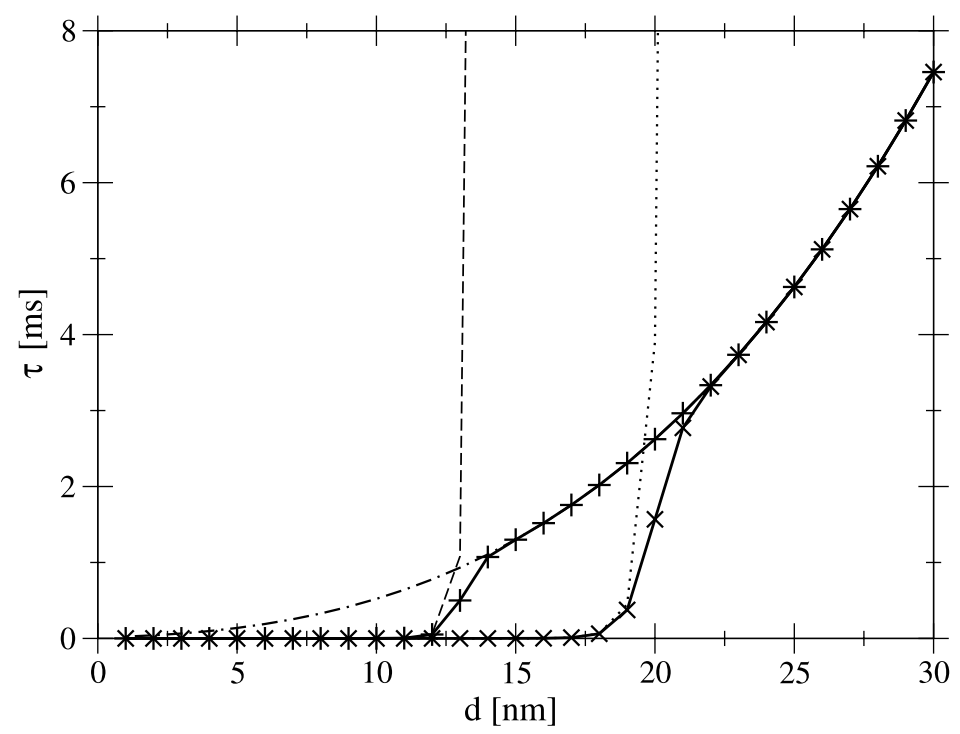

Fig. 16. Relaxation times as a function of particle diameter $d$ : Brownian (dot-dashed line), Néel (dotted line for $K=15 \mathrm{~kJ} / \mathrm{m}^{3}$, dashed line for $K=50 \mathrm{~kJ} / \mathrm{m}^{3}$ ), and the combination (53) (solid line with crosses for $K=15 \mathrm{~kJ} / \mathrm{m}^{3}$, solid line with plusses for $K=50 \mathrm{~kJ} / \mathrm{m}^{3}$ ).

amplitude correction factor $f$ discussed below that forces the model prediction for the maximal $H_{y}^{\text {sensor }}$ to coincide with the measured one. Figure 17 shows the comparisons of experimental data with the fits for the case of the medium sized field $H_{0}=30 \mathrm{kA} / \mathrm{m}$. The parameters used to calculate $H_{y}(\Omega)$ are summarized in Table 5. We furthermore used as input the experimental equilibrium magnetization $M_{\mathrm{eq}}(H)$ of APG 933, the experimental initial susceptibility $\chi_{0}=1.09$ and the vortex viscosity $\zeta=\frac{3}{2} \Phi \eta$ with viscosity $\eta=0.5 \mathrm{~Pa}$ s and volume concentration $\Phi=0.041$.

The necessity of an amplitude reduction factor $f<1$ reflects in our opinion the fact that only a fraction of the particles contribute to the experimental signal: only those particles contribute that have a volume large enough to ensure the magnetic moment to be fixed within the particle and therefore to relax in a Brownian manner.

In the experiments done with polydisperse ferrofluids for frequencies up to $\Omega \simeq 3000 \mathrm{rad} / \mathrm{s}$ mainly the large particles contribute to $M_{y}$ resp. to $H_{y}^{\text {sensor }}$ since their magnetic moments are effectively frozen in the particle's crystal lattice. Only these magnetic moments can be pushed out of the direction of the magnetic field by the combined action of thermally induced rotary Brownian motion and deterministic macroscopic flow in the rotating cylinder. Smaller particles can keep their moment aligned with the magnetic field by the Néel process when these particles undergo rotational motion. Finally, the particle 


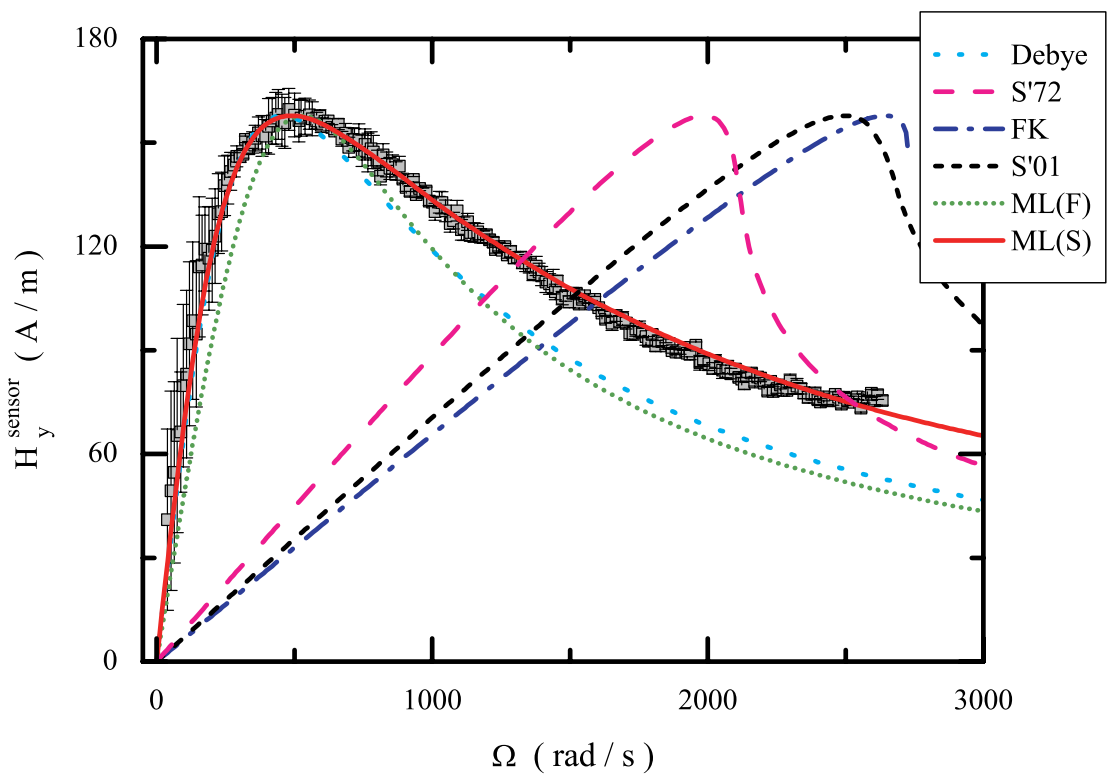

Fig. 17. Comparison of the experimental data (symbols) obtained at $H_{0}=30 \mathrm{kA} / \mathrm{m}$ with the numerical results according to different theoretical models. See Table 5 for an identification of the curves and the fit parameters used.

Table 5. Models described in Sect. 4.2.1 are used in Fig. 17 in order to get the presented curves. $f$-factor refers to the amplitude factor.

\begin{tabular}{llcc}
\hline model & color & $f$-factor & relaxation time $\tau[\mathrm{ms}]$ \\
\hline Debye & (dotted) & 0.164 & 2.5 \\
S'72 & (dashed) & 0.164 & 2.5 \\
FK & (dashed dotted) & 0.125 & 5 \\
S'01 & (short dashed) & 0.125 & 3.5 \\
ML(F) & (short dotted) & 0.125 & 6 \\
ML(S) & (solid) & 0.125 & 3.5 \\
\hline
\end{tabular}

diameter that separates Néel behavior from Brownian behavior in the size distribution and that thereby determines how many particles contribute to $M_{y}$ resp. to the experimental signal $H_{y}^{\text {sensor }}$ depends sensitively on the anisotropy constant $K$ : The smaller $K$, the smaller is the number of Brownian particles according to Fig. 16, and the smaller is $M_{y}$ resp. $H_{y}^{\text {sensor }}$.

The above sketched physical picture is corroborated by Fig. 18. There we compare the experimentally obtained $H_{y}^{\text {sensor }}(\Omega)$ (stars) as a function of $\Omega$ for a representative externally applied field $H_{0}=30 \mathrm{kA} / \mathrm{m}$ with various 


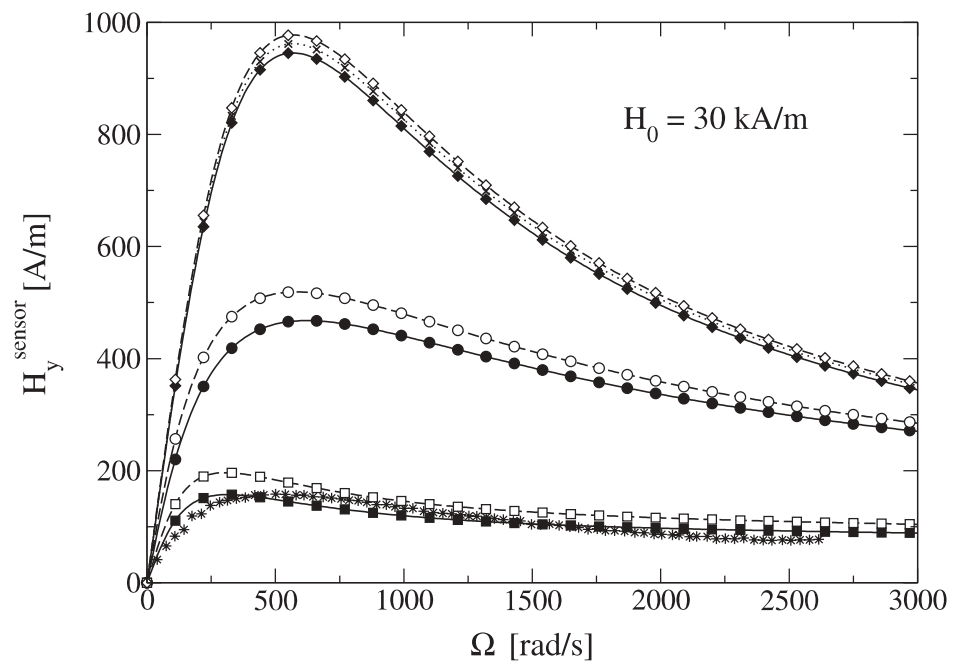

Fig. 18. $H_{y}^{\text {sensor }}$ as function of $\Omega$ for $H_{0}=30 \mathrm{kA} / \mathrm{m}$. Experimental data for APG 933 (stars) are compared with the results of different theoretical models. The three uppermost curves refer to single-time relaxation approximations, each with $\tau=2 \mathrm{~ms}$ : monodisperse Debye model (dotted line with crosses), polydisperse models with common $\tau_{j}=\tau$ and magnetic weights $w_{j}$ obtained with a lognormal distribution (full line with full diamonds) and with a regularization method (dashed line with open diamonds). Curves with circles and squares refer to truly polydisperse models (Eqs. (52-53)) with $K=50 \mathrm{~kJ} / \mathrm{m}^{3}$ (circles) and $K=15 \mathrm{~kJ} / \mathrm{m}^{3}$ (squares). Full lines with full symbols were obtained with a lognormal distribution. Dashed lines with open symbols refer to a distribution resulting from the regularization method. In all models the equilibrium magnetization $M_{\mathrm{eq}}(H)$ was taken to be the experimental one.

model variants that take into account the polydispersity to different degrees. This is done for two different anisotropy constants, namely, $K=15 \mathrm{~kJ} / \mathrm{m}^{3}$ and $K=50 \mathrm{~kJ} / \mathrm{m}^{3}$ as representative examples. However, the three uppermost curves refer to single time relaxation approximations, each with $\tau=2 \mathrm{~ms}$ : the dotted line with crosses is the result of the strictly monodisperse Debye model used above, however without amplitude correction factor $(f=1)$. The lines with diamonds refer to polydisperse models, however, with common $\tau_{j}=\tau=2 \mathrm{~ms}$ taken in Eq. (52) but different magnetic weights $w_{j}$ obtained either from a lognormal distribution (full line with full diamonds) or from the regularization method (dashed line with open diamonds). The equilibrium magnetization $M_{\mathrm{eq}}(H)$ was taken to be the experimental one, the distributions were obtained from this experimental $M_{\mathrm{eq}}(H)$ by the lognormal ansatz resp. the regularization method. This, first of all, shows that models with only one relaxation time show roughly the same behavior of $M_{y}(\Omega)$ irrespective of whether the particle size and magnetic moment distributions are polydisperse or not. 
The set of curves with circles and squares in Fig. 18 refer to truly polydisperse models, Eqs. (52-53), but different anisotropy constants of the magnetic material $\left(K=50 \mathrm{~kJ} / \mathrm{m}^{3}\right.$ (circles), $K=15 \mathrm{~kJ} / \mathrm{m}^{3}$ (squares)). For the numerical calculations we used typical values for the ferrofluid APG 933: $M_{\text {sat }}^{\text {bulk }}=$ $456 \mathrm{kA} / \mathrm{m}, \eta=0.5 \mathrm{Pas}, s=2 \mathrm{~nm}$, and $f_{0}=10^{9} \mathrm{~Hz}$. Again, full lines with full symbols were obtained with a lognormal distribution while dashed lines with open symbols refer to a distribution resulting from the regularization method.

The largest and most important difference between the curves with diamonds (i.e., the single-time models) and the curves with circles and squares (i.e., the genuine polydisperse models) come from the difference in the anisotropy constants of the magnetic material that governs how many particles contribute efficiently as Brownian ones to the transverse magnetization $M_{y}$ resp. to $H_{y}^{\text {sensor: }}$ for smaller $K$ the magnetic moments of fewer particles being Brownian ones may be rotated out of the direction of the magnetic field by the flow in the cylinder. The curves for $K=15 \mathrm{~kJ} / \mathrm{m}^{3}$ yield roughly the same maximal size $H_{y}^{\text {sensor }}$ as the experiments - they could be fine-tuned even further.

Furthermore we found that the models FK, S'01, and S'72 do not properly reproduce the position $\Omega^{\max }$ of the maximum of the experimental curve in Fig. 17. In contrast, using the other models with an appropriate choice of the fit-parameter $\tau$ we can reproduce the peak position $\Omega^{\max }$. However, only the model ML(S) reproduces the experimental curve in Fig. 17 for larger values of $\Omega$.

From the measured curves of $H_{y}\left(\Omega, H_{0}\right)$ versus $H_{0}$ we extracted the position of the maximum (denoted as $\Omega^{\max }\left(H_{0}\right)$ ) and the maximum value $\max \left(H_{y}^{\text {sensor }}\right)=H_{y}^{\text {sensor }}\left(\Omega^{\max }, H_{0}\right)$. The latter is shown in Fig. 19 in comparison with the results of the models. In order to fit the maximum amplitude we had to introduce an $a d$-hoc additional amplitude reduction factor $f$ ( $c f$. Table 5). Note that the models Debye and S'72 yield the same maximum (Sect. 4.2.1). The other models [FK, S'01, ML(F), and ML(S)] also have a common but different maximal $H_{y}$. Thus, Table 5 contains only two different amplitude reduction factors $f$. Fig. 19 shows that already the simple Debye model reasonably well reproduces the variation of $\max \left(H_{y}^{\text {sensor }}\right)$ with $H_{0}$ if one ignores the $a d$-hoc amplitude reduction factor.

We have seen, that only the polydisperse model can reproduce the amplitude $\max \left(H_{y}^{\text {sensor }}\right)$ without an amplitude reduction factor. But also above polydisperse model yields locations, $\Omega^{\max }\left(H_{0}\right)$, of the maxima for different $H_{0}$ which are still off from the experimental ones as shown in Fig. 20a and 21b. However, the agreement between the predictions of the polydisperse models of Eqs. (52-53) and the experiments concerning the location $\Omega^{\max }\left(H_{0}\right)$ can be improved by allowing the relaxation rates $\tau_{j}$ of the differently sized particles to depend also on the internal field $H$. We used the ansatz [42]

$$
\tau_{j}^{\gamma}(H)=\tau_{j} \frac{2 \mathcal{L}(\gamma H)}{\gamma H-\mathcal{L}(\gamma H)},
$$




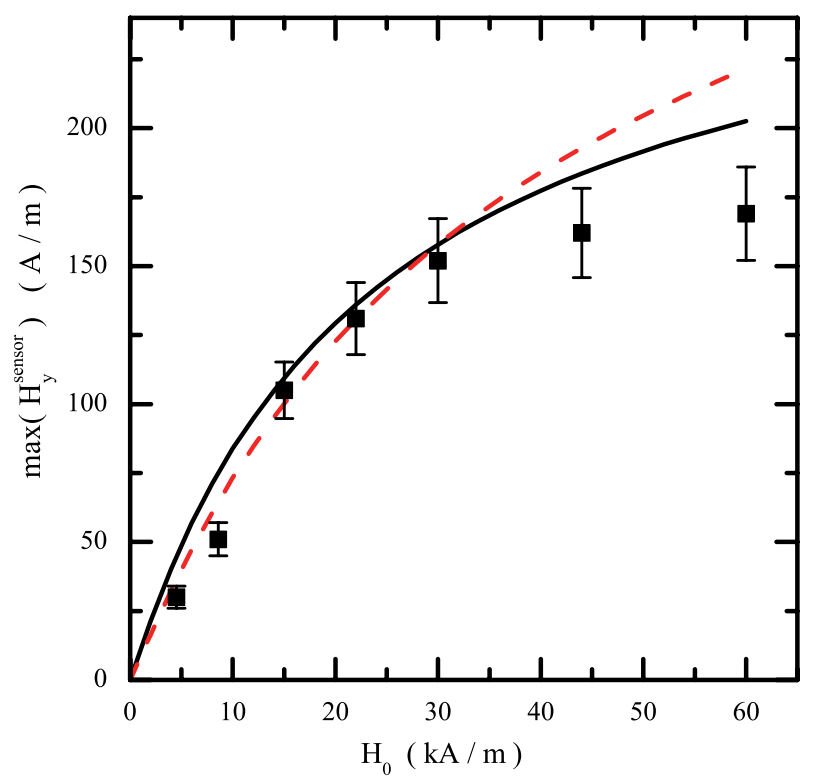

Fig. 19. Maxima of $H_{y}^{\text {sensor }}=H_{y}^{\text {sensor }}\left(\Omega^{\max }\right)$ as a function of the external field $H_{0}$. Squares with error bars refer to experimental data. Solid line shows the result of the Debye and S'72 model. Dashed line is common to the models denoted as FK, S'01, and ML. Parameters are listed in Table 5.

with one additional fit parameter $\gamma$. Values of about $\gamma \sim 10^{-4} \mathrm{~m} / \mathrm{A}$ yield maximum locations $\Omega^{\max }\left(H_{0}\right)$ that agree well with the experiments as can be seen in Fig. 20b and 21b. This generalization of the model (52-53) leaves the peak value $H_{y}^{\text {sensor }}\left(\Omega^{\max }\right)$ almost unchanged, $c f$. Fig. 21a.

However, also this augmented polydispersive model reproduces with fixed values of $K$ and $\gamma$ the experimental data only in a small range of $\Omega$ and $H_{0}$, cf. Figs. 20 and 21.

\subsection{Conclusion}

We have compared the predictions of polydisperse models of the magnetization dynamics of ferrofluids with recent experiments measuring the transverse magnetization component $M_{y}$ of a rotating ferrofluid cylinder. The models use mixtures of mono-disperse ideal paramagnetic gases of differently sized particles. The magnetization dynamics of the models take into account the rigid body rotation of the fluid combined with a simple Debye relaxation of the magnetic moments of each particle with size dependent Brownian and Néel magnetic relaxation times. Thus, in the absence of flow, each magnetic moment and with it each sub-magnetization would be driven independently of the others towards its respective mean equilibrium value that, however, depends 


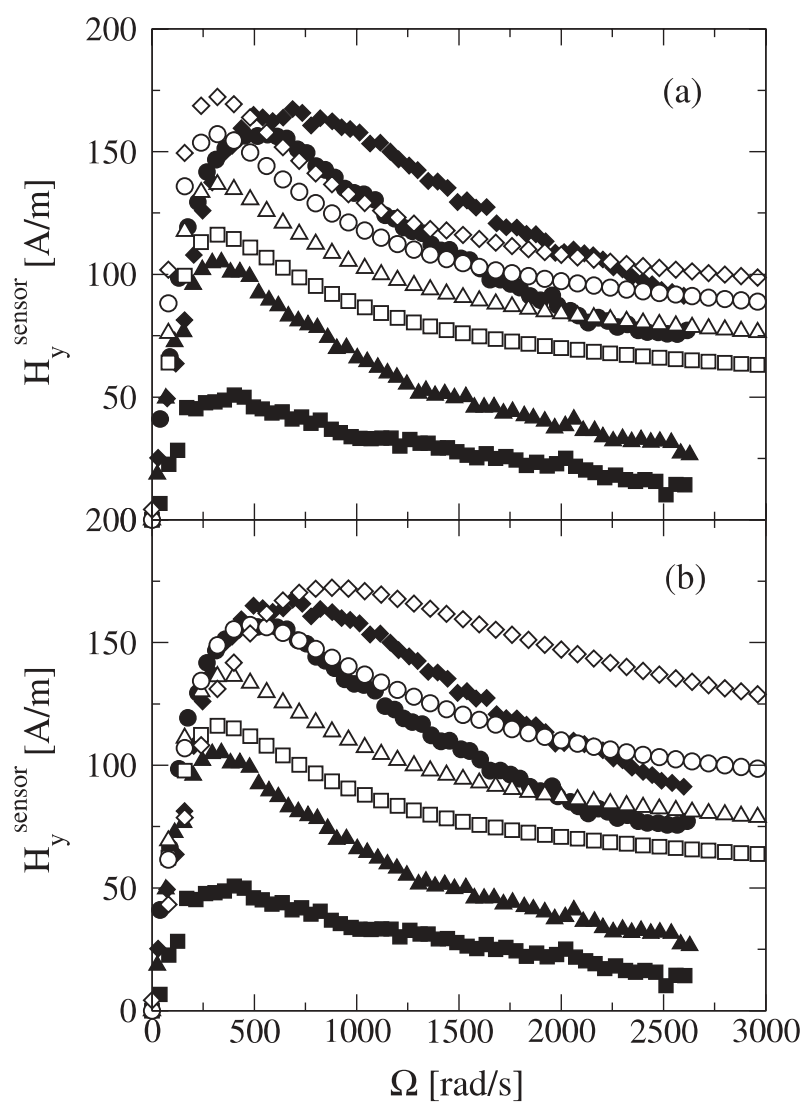

Fig. 20. $H_{y}^{\text {sensor }}$ as function of $\Omega$ for $H_{0}=8.6 \mathrm{kA} / \mathrm{m}$ (squares), $15 \mathrm{kA} / \mathrm{m}$ (triangles), $30 \mathrm{kA} / \mathrm{m}$ (circles), and $60 \mathrm{kA} / \mathrm{m}$ (diamonds). Full symbols denote experimental data. Open symbols refer to the polydisperse model (Eqs. (52-53)) with lognormal distribution and $K=15 \mathrm{~kJ} / \mathrm{m}^{3}$ : in (a) the relaxation times $\tau_{j}$ are independent of $H$, in (b) they are replaced by $\tau_{j}^{\gamma}(H)$ (54) with $\gamma=10^{-4} \mathrm{~m} / \mathrm{A}$.

on the internal magnetic field $\boldsymbol{H}$ being collectively generated by all magnetic moments.

The comparison suggests that mainly the large particles contribute to $M_{y}$ since their magnetic moments are effectively frozen in the particle's crystal lattice. Smaller particles can keep their moment aligned with the magnetic field by the Néel process when these particles undergo rotational motion.

Finally, the particle diameter that separates Néel behavior from Brownian behavior in the size distribution and that thereby determines how many particles contribute to $M_{y}$ resp. to the experimental signal $H_{y}^{\text {sensor }}$ depends quite sensitively on the anisotropy constant $K$ of the magnetic material. $K$ deter- 


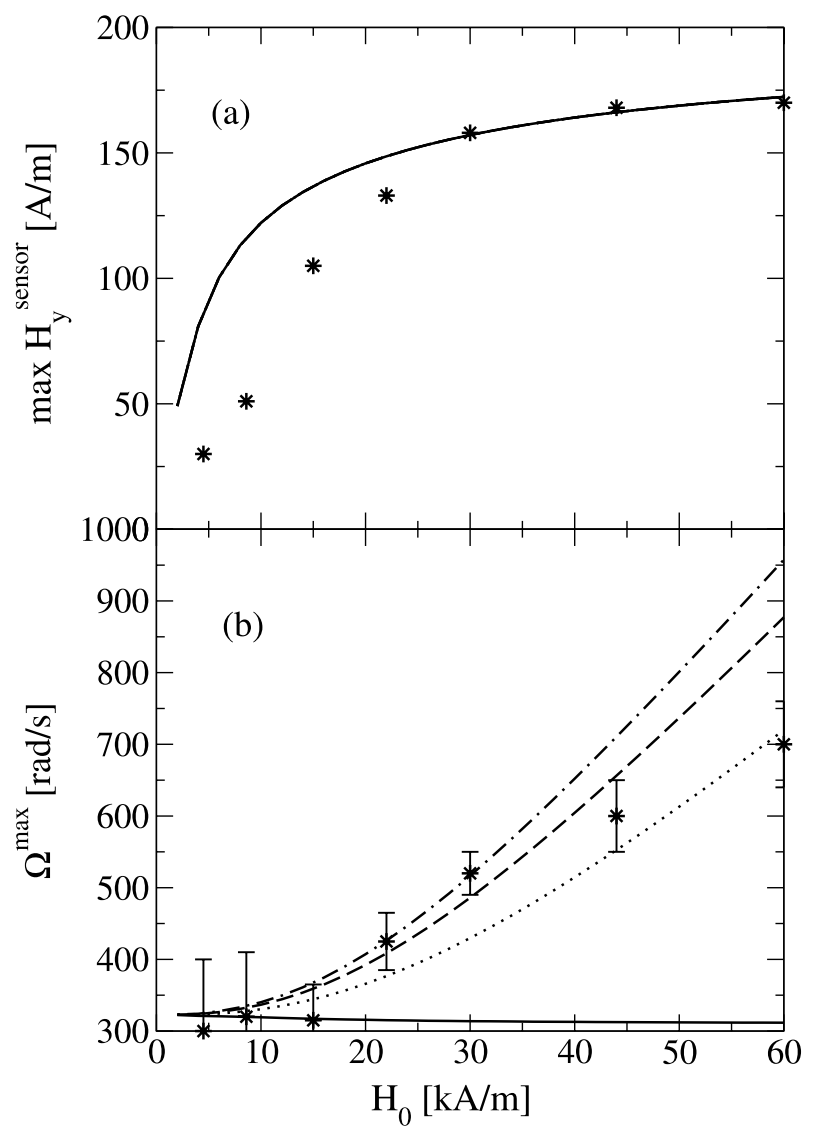

Fig. 21. Maximum magnitude $\max \left(H_{y}^{\text {sensor }}\right)=H_{y}^{\text {sensor }}\left(\Omega^{\max }\right)$ (a) and location of the maximum $\Omega^{\max }$ (b) as functions of the external field $H_{0}$. Stars show experimental data. Lines refer to the results of polydisperse models with a lognormal distribution and $K=15 \mathrm{~kJ} / \mathrm{m}^{3}: H$-independent relaxation times (solid); $H$-dependencies with $\gamma=0.8 \times$ $10^{-4} \mathrm{~m} / \mathrm{A}$ (dotted), $\gamma=10^{-4} \mathrm{~m} / \mathrm{A}$ (dashed), and $\gamma=1.1 \times 10^{-4} \mathrm{~m} / \mathrm{A}$ (dot-dashed). In (a) the differences between the lines are too small to be seen.

mines how many magnetic moments are "frozen" or "blocked" in particles and thus can be rotated by the rigid body flow: The smaller $K$, the smaller is the number of Brownian particles with frozen moments, and the smaller is the resulting $M_{y}$. Or, vice versa, a large transverse magnetization can be expected in ferrofluids with large anisotropy constants.

An analysis of the rotation rates $\Omega^{\max }\left(H_{0}\right)$ for which $M_{y}$ is largest indicates that the agreement between experiments and model predictions can be improved by allowing the relaxation rates of the differently sized particles to depend also on the magnetic field $H$. 


\section{Surface instabilities}

Here we discuss and compare results from experimental investigations of the anomalous dispersion branch of surface waves on ferrofluids in a magnetic field and numerical analyses [61]. In our experiments we used the well known Faraday instability of parametrically excited surface waves on a ferrofluid in the presence of a magnetic field oriented perpendicular to the fluid's surface.

\subsection{Earlier work}

Cowley and Rosensweig [62] have investigated experimentally the instability of a flat ferrofluid surface in a static magnetic field $H$ oriented perpendicular to the surface: above a certain critical value, $H_{\mathrm{C}}^{\mathrm{R}}$, the surface spontaneously deforms into regularly spaced peaks, that are usually arranged in a hexagonal pattern. At the critical field $H_{\mathrm{C}}^{\mathrm{R}}$ of this so-called Rosensweig instability, the dispersion curve of surface waves touches the zero frequency axis at a finite wave number $k_{\mathrm{C}}^{\mathrm{R}}$, analogous $e . g$. to the soft mode behavior of phonons at structural phase transitions of solids. For magnetic fields slightly below $H_{\mathrm{C}}^{\mathrm{R}}$, the dispersion is non-monotonous with a section of negative slope (anomalous dispersion). There exists a number of papers dealing with theoretical modelling of the dispersion relation [63-66]. But up to now the anomalous dispersion has not been detected experimentally, although there is experimental evidence for a jump in wave number [67] that is expected to occur in the presence of anomalous dispersion when ramping the frequency up and down [68].

The excitation of surface waves has been realized by different mechanisms. Pétrélis et al. [69] have studied the influence of the Faraday instability mechanism of parametrically excited surface waves that arise in vertically shaken containers on the Rosensweig instability for driving frequencies $f \geq 30 \mathrm{~Hz}$. They have demonstrated experimentally that the Faraday instability inhibits the Rosensweig instability, a fact that was predicted by Müller in [68]. In [67] Mahr et al. have reported the existence of two different wave numbers occurring at the same excitation frequency in a V-shaped annular teflon channel. In order to generate surface waves they have directed an air jet towards the magnetic fluid's surface. Results of measurements at a magnetic field $3 \%$ below the threshold of the Rosensweig instability have been presented but the anomalous region of the dispersion has not been studied. Due to the finite geometry of the vessel a quantitative comparison of their experimental results with calculation was not possible. In Ref. [70] a mechanical excitation of surface waves in the presence of a dc magnetic field was used. The authors have found so-called twin-peak patterns in the non-monotonic regime of the dispersion relation $2 \%$ below the critical field $H_{\mathrm{C}}^{\mathrm{R}}$ and furthermore domain structures, oscillating defects and relaxational phase oscillations. These findings have been attributed to the non-monotonic dispersion relation of ferrofluids. The same group has presented in [71] experimental results on surface waves, that have been excited 
by an oscillating magnetic field. Both, subharmonic and harmonic surface response have been detected. Higher order resonance tongues were generated by tuning the viscous damping with an external magnetic field. This was made possible by a specific peculiarity of ferrofluids, namely a very weak damping of surface waves when the wave number is close to $k_{\mathrm{C}}^{\mathrm{R}}$. Browaeys et al. [72] have used a small coil placed beneath the ferrofluid container in order to generate surface waves. With this method it was very difficult to excite surface waves with wavelengths small compared to the coils diameter. They limited the ratio $H / H_{\mathrm{C}}^{\mathrm{R}}$ to 0.79 because above this ratio the peaks started to form at the boundary of the vessel.

\subsection{Generating surface waves with anomalous dispersion}

A drawback of generating surface waves by modulating the magnetic field is that the time variation of the magnetization $M(t)=\chi H(t)$ enters quadratically into the dispersion relation so that the effective excitation signal is composed of two frequencies. Furthermore, the effective magnetic drive is proportional to $k^{2}$ while it is linear in $k$ for the gravity modulation. Thus, shorter wavelength are favored by the magnetic drive. To avoid these complications the Faraday forcing is used, where surface waves are generated only beyond a critical excitation amplitude [73]. In this type of experiment the earth's gravity acceleration $g_{0}$ is modulated in the co-moving frame according to $g(t)=g_{0}+a \cos (2 \Omega t)$. As long as the amplitude $a$ is smaller than a critical one, $a_{\mathrm{C}}$, the surface of the ferrofluid remains flat. By increasing $a$ beyond $a_{\mathrm{C}}$, the flat surface becomes unstable and the system forms standing waves. The wave pattern depends on the mass density $\rho$, surface tension $\sigma$, viscosity $\eta$ of the fluid and its filling level $h$. For small values of $h$, an experimental investigation of the anomalous part of the dispersion relation was predicted to be feasible [68].

To get access to the anomalous dispersion branch we took advantage of the dissipation in the bottom boundary layer [68] that is responsible for the suppression of the growth of waves with wave numbers below $k \sim 1 / h=200 \mathrm{~cm}^{-1}$ for $h=5 \mathrm{~mm}$. Being strongly damped the onset of such waves in the region of linear dispersion is delayed. Furthermore, by choosing the viscosity appropriately the marginal stability curve $a(k)$ of the flat surface against the growth of standing waves can be tuned such that its absolute minimum $k_{\mathrm{c}}$ lies in the wave number region of anomalous dispersion. Then the growth rates of waves in this region are largest and get selected when increasing the acceleration above threshold.

Besides the filling level $h$, the fluid's viscosity $\eta$ influences the marginal curves in a way, that can be used to get access to the anomalous dispersion branch. The influence of the viscosity on the marginal curves is shown in Fig. 22. The upper panel of Fig. 22 shows the marginal curves for a ferrofluid of low viscosity $(\eta=6.66 \mathrm{mPa}$ s), while in the lower panel the high-viscosity case $(\eta=53.3 \mathrm{mPa} \mathrm{s})$ is presented. In the latter, the high viscosity clearly favors the 


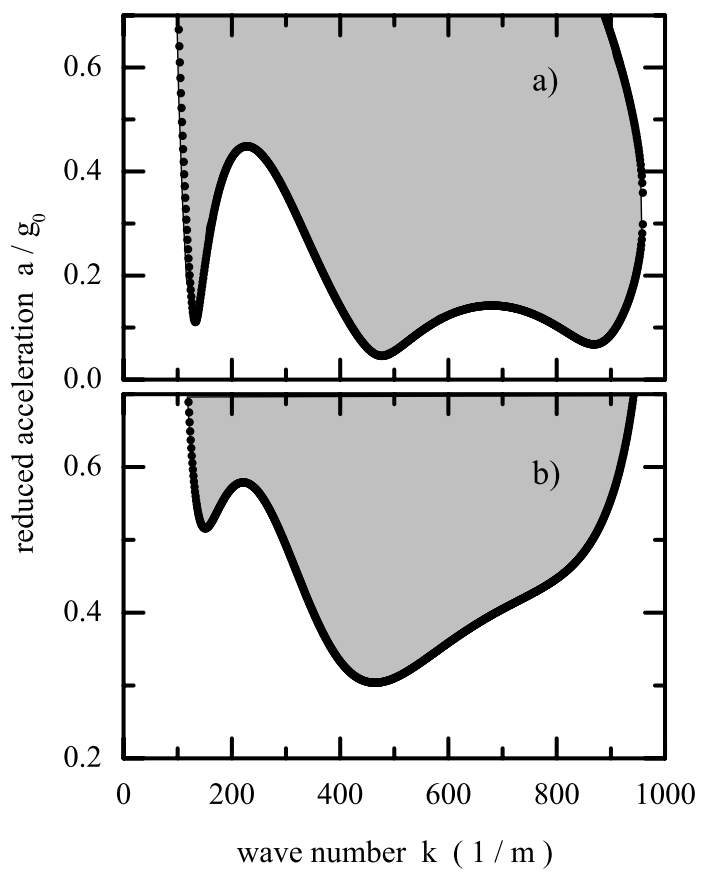

Fig. 22. Marginal stability curves of the flat surface against growth of standing waves for different values of the viscosity $\eta$ (a) $\eta=6.66 \mathrm{mPa} \mathrm{s}$, (b) $\eta=53.3 \mathrm{mPa}$ ) for the following parameters: $f=7 \mathrm{~Hz}, h=5 \mathrm{~mm}, \sigma=0.022 \mathrm{~N} / \mathrm{m}, \rho=1110 \mathrm{~kg} / \mathrm{m}^{3}, \chi=0.8$ and $M=0.999 M_{\mathrm{C}}^{\mathrm{R}}$. Within the gray regions, the plane surface is unstable.

local minimum belonging to the anomalous dispersion branch. Thus, choosing an appropriate combination of filling level and fluid's viscosity enabled us to see in our experiment only the dispersion branch of interest, i.e. the anomalous one.

\subsection{Experimental setup}

The experimental setup is sketched in Fig. 23. The cylindrical container filled with the ferrofluid is attached to a mechanical vibrator (Large Displacement Shaker V617, Gearing \& Watson Electronics Ltd.) connected to a $4 \mathrm{~kW}$ power amplifier (DSA-4k, Gearing \& Watson Electronics Ltd.). The container is placed in the center of a pair of Helmholtz coils providing a magnetic field oriented perpendicular to the fluid surface. The homogeneity of the field across the liquid level is better than 5\%. The sinusoidal signal for the power amplifier was synthesized by a digital-analog card installed in a Pentium PC. The vertical acceleration of the container is measured by a piezo-electric device (Brüel \& Kjaer 4393). The signal of this device is amplified (Brüel \& Kjaer 2525) 


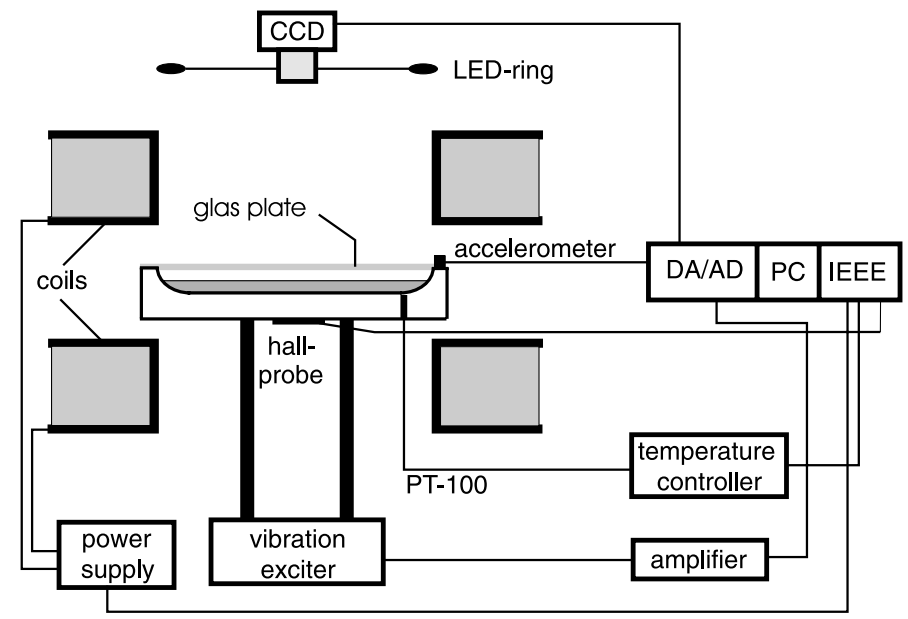

Fig. 23. Sketch of the experimental setup. For further details see text.

and routed to the PC for data acquisition. The temperature was regulated to $T=(24.5 \pm 0.1) \mathrm{K}$ to hold the viscosity and the surface tension of the liquid constant. In order to visualize the surface structure a full frame CCD camera (Hitachi KPF-1) was placed about $1 \mathrm{~m}$ above the interface ferrofluid/air in the center of a ring (with radius $r=0.3 \mathrm{~m}$ ) of 120 LEDs illuminating the surface. Using this visualization technique only surface elements of appropriate slope reflect light into the camera, while the flat regions of the surface appear black. The spatial symmetry of the surface deformation $\zeta(x, y)$ was analyzed by using two-dimensional Fourier transformation. For the measurements we used a commercial ferrofluid (APG J12, FerroTec) with the following specifications: dynamic viscosity $\eta=45 \times 10^{-3} \mathrm{~Pa} \mathrm{~s}$, mass density $\rho=1110 \mathrm{~kg} / \mathrm{m}^{3}$ and initial magnetic susceptibility $\chi=0.8$.

\subsection{Dispersion relation}

The dispersion relation of surface waves for an ideal inviscid fluid in a perpendicular magnetic field,

$$
\begin{aligned}
\omega_{0}^{2}(k)= & \tanh (k h)\left\{g_{0} k+\frac{\sigma}{\rho} k^{3}-\frac{\mu_{0}}{\rho}\left(\frac{1+\chi}{2+\chi}\right)\right. \\
& \left.\times\left[1-\frac{2 \chi}{(2+\chi)^{2} \mathrm{e}^{2 k h}-\chi^{2}}\right] M^{2} k^{2}\right\},
\end{aligned}
$$

depends on $h, g_{0}, \sigma, \chi$ and the magnetization $M$. For high filling levels, $k h \gg 1$, the critical magnetization $M_{\mathrm{C}}^{\mathrm{R}}$ and the critical wave number $k_{\mathrm{C}}^{\mathrm{R}}$ of the 
Rosensweig instability read

$$
\left(M_{\mathrm{C}}^{\mathrm{R}}\right)^{2}=\frac{2}{\mu_{0}}\left(\frac{2+\chi}{1+\chi}\right) \sqrt{\rho g_{0} \sigma}, \quad k_{\mathrm{C}}^{\mathrm{R}}=\sqrt{\rho g_{0} / \sigma} .
$$

There is a second characteristic value of the magnetization $\tilde{M}<M_{\mathrm{C}}^{\mathrm{R}}$ above which the dispersion curve is no longer monotonic. A simple calculation gives: $\widetilde{M}=\sqrt[4]{3 / 4} M_{\mathrm{C}}^{\mathrm{R}}$, i.e., $\widetilde{M} \approx 0.93 M_{\mathrm{C}}^{\mathrm{R}}$. For $M>\widetilde{M}$ the dispersion relation $\omega_{0}^{2}(k)$ shows a minimum so that there is a range of $k$-values in which the group velocity is negative; this is referred to as anomalous dispersion. Thus, for $M$ approaching $M_{\mathrm{C}}^{\mathrm{R}}$ there is a band of driving frequencies in which surface waves with three different $k$-values can be generated.

For real fluids the viscosity of the fluid must be taken into account. Using the expression of Kumar and Tuckerman [73] the dispersion relation subject to appropriate boundary conditions reads now

$$
-\omega^{2}+\omega^{2} X(k, \omega)+\omega_{0}^{2}(k)=0 .
$$

The magnetization enters via the inviscid dispersion relation $\omega_{0}(k)(55)$ so that

$$
\begin{aligned}
& -\omega^{2}+\omega^{2} X(k, \omega)=\frac{v^{2}}{q \operatorname{coth}(q h)-k \operatorname{coth}(k h)} \\
& \times\left\{q\left[4 k^{4}+\left(k^{2}+q^{2}\right)^{2}\right] \operatorname{coth}(q h)-k\left[4 k^{2} g^{2}+\left(k^{2}+q^{2}\right)^{2}\right] \tanh (k h)-\right\} . \\
& \left\{\cdot \frac{4 k^{2} q\left(k^{2}+q^{2}\right)}{\cosh (k h) \sinh (q h)}\right\},
\end{aligned}
$$

with $q=\sqrt{k^{2}+\mathrm{i} \omega / \nu}$, where $v$ denotes the kinematic viscosity. In order to determine the critical parameters $a_{\mathrm{C}}$ and $k_{\mathrm{C}}$ of the Faraday instability for a given field value $H$ and a given driving frequency $f$, we used a special version of the algorithm proposed by Kumar and Tuckerman. The surface deformation is described by a Floquet-ansatz

$$
\zeta \propto \mathrm{e}^{\mathrm{i} \beta \Omega t} \sum_{n=-\infty}^{\infty} \zeta_{n} \mathrm{e}^{2 \mathrm{i} n \Omega} .
$$

Here $\beta=1(\beta=0)$ corresponds to a subharmonic (harmonic) response of the surface deformation respectively. The Floquet-ansatz leads to tridiagonal system of equations

$$
0=\left[-\omega^{2}+\omega^{2} X(\omega, k)+\omega_{0}^{2}(k)\right] \zeta_{n}+\frac{a k \tanh (k h)}{2}\left(\zeta_{n+1}+\zeta_{n-1}\right),
$$

with $\omega=(2 n+\beta) \Omega$. Eqs. (61) can be numerically approximated by an appropriate cutoff. The solvability condition yields the neutral stability curves 


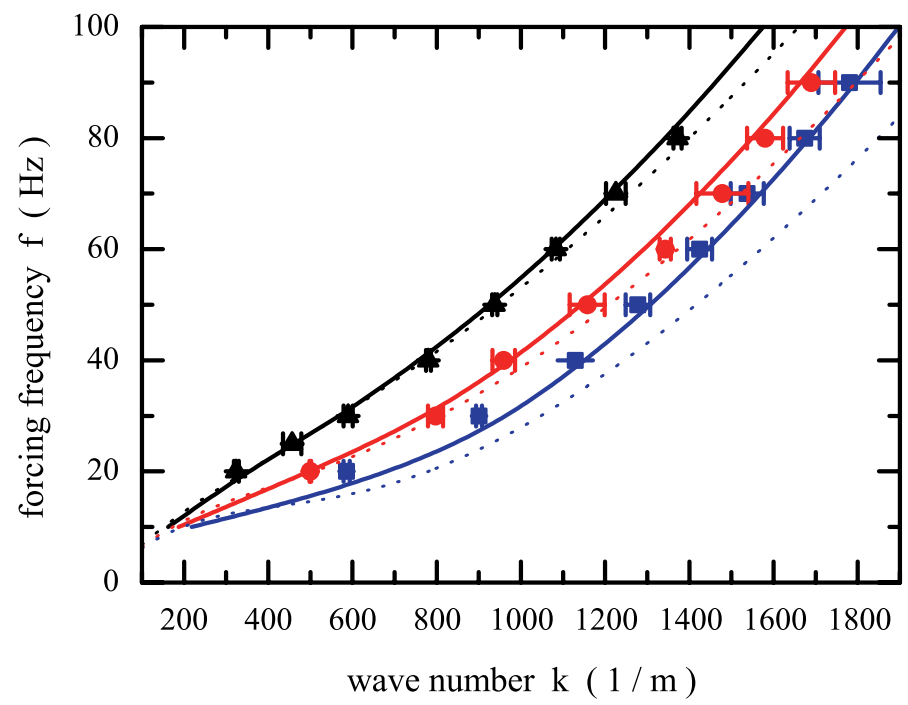

Fig. 24. Forcing frequency $f$ versus wave number $k$ of the standing wave pattern at slightly supercritical acceleration amplitudes. The solid lines show critical values resulting from Eqs. $(57,58,60,61)$ with the parameters $\rho=1110 \mathrm{~kg} / \mathrm{m}^{3}, \chi=0.8, \sigma=0.02 \mathrm{~N} / \mathrm{m}$ and $\eta=45.77 \times 10^{-3} \mathrm{~Pa}$ s. The dashed lines are calculated for $\eta=0$. The magnetization was $M=0,0.7 M_{\mathrm{C}}^{\mathrm{R}}$, and $0.87 M_{\mathrm{C}}^{\mathrm{R}}$ from top to bottom, respectively.

$a^{\mathrm{SH}}(k)$ and $a^{\mathrm{H}}(k)$ for the subharmonic $(\mathrm{SH})$ and harmonic $(\mathrm{H})$ surface response, respectively. A minimization of these curves with respect to the wave number $k$ determines the critical acceleration $a_{\mathrm{C}}^{\mathrm{SH}}\left(a_{\mathrm{C}}^{\mathrm{H}}\right)$ and the critical wave number $k_{\mathrm{C}}^{\mathrm{SH}}\left(k_{\mathrm{C}}^{\mathrm{H}}\right)$.

\subsection{Experimental and numerical results}

Here we first present results of experiments performed at a fixed filling level of $h=5 \mathrm{~mm}$. The surface tension $\sigma$ of the ferrofluid was not known, therefore we first performed measurements at $M=0, M=0.7 M_{\mathrm{C}}^{\mathrm{R}}$ and $M=0.87 M_{\mathrm{C}}^{\mathrm{R}}$. The data of these measurements are presented in Fig. 24. As demonstrated in $[73,74]$ the Faraday instability provides a versatile method to measure the surface tension. With our investigations we measured the critical acceleration $a_{\mathrm{C}}$ as function of the driving frequency. Furthermore, the critical wave number $k_{\mathrm{C}}$ was determined from video images recorded close to the threshold of the Faraday instability.

Our experimental results are well described by numerical calculations using Eqs. (57-61) and the following parameters: $\eta=45.77 \times 10^{-3} \mathrm{Pas}, \chi=$ 0.8 and $\sigma=0.02 \mathrm{~N} / \mathrm{m}$. So the surface tension $\sigma=0.02 \mathrm{~N} / \mathrm{m}$ is a direct outcome of our experiments. In order to study the anomalous dispersion branch 


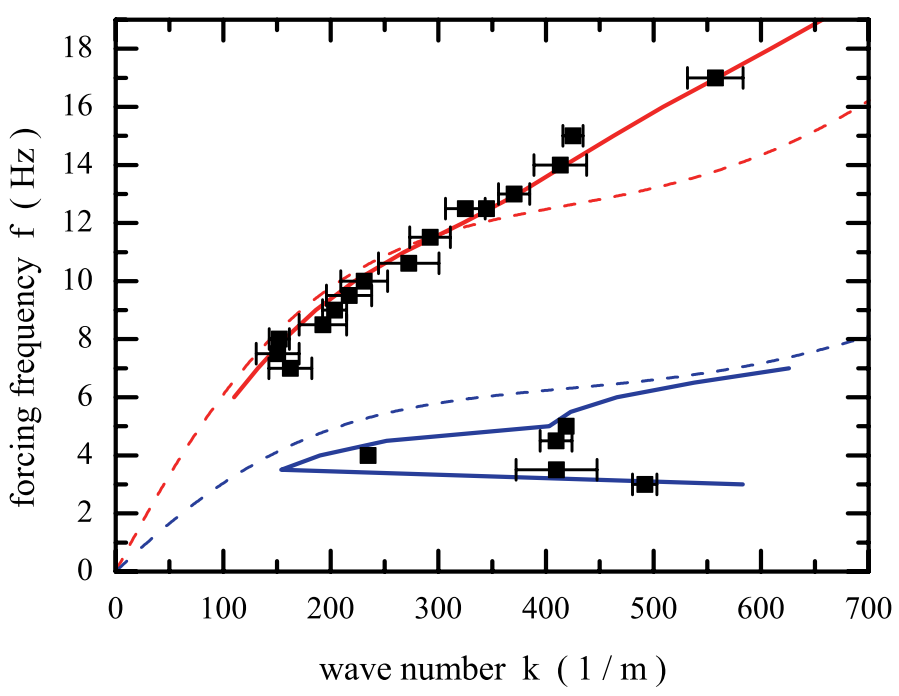

Fig. 25. Forcing frequency $f$ versus wave number $k$ as in Fig. 24, here, however, for $M=0.9 M_{\mathrm{C}}^{\mathrm{R}}$. For $3 \mathrm{~Hz} \leq f \leq 5.5 \mathrm{~Hz}$ the surface response is harmonic in time, while for $f \geq 6 \mathrm{~Hz}$ it is subharmonic. Full lines show critical values as in Fig. 24; dashed curves refer to the idealized case $\eta=0$.

the external field strength was chosen to be shortly below the critical value. The experiments have been performed in the following way: with $M$ and $f$ set to the desired values, the acceleration of the shaker has been slowly increased up to the critical value of the Faraday instability where the initially flat surface transforms into a standing wave pattern. In order to map the surface deformation we had to increase the acceleration further to about $3-5 \%$ above $a_{\mathrm{c}}$.

In Fig. 25 we present the experimental results obtained at $M=0.9 M_{\mathrm{R}}^{\mathrm{C}}$. For forcing frequencies $3 \mathrm{~Hz} \leq f \leq 5.5 \mathrm{~Hz}$ the system responds with a frequency equal to the forcing frequency (harmonic response), while for $f \geq 6 \mathrm{~Hz}$ the response frequency is half the driving frequency (subharmonic response). In contrast to the subharmonic dispersion curve, that shows normal behavior, the harmonic one in the lower part of Fig. 25 is more complex which is well described by the numerical results. We stress that in non-magnetizable fluids temporal harmonic surface waves are realizable only either at very extreme experimental conditions, i.e., low excitation frequencies and very low filling levels (usually below $h=1 \mathrm{~mm}$ ) $[75,76]$ or by using non-Newtonian liquids [77].

In a second experiment $M$ was set $1.3 \%$ below the critical Rosensweig value $M_{\mathrm{C}}^{\mathrm{R}}$ (see Fig. 26). Both, the temporal harmonic and the temporal subharmonic dispersion relation now show anomalous behavior for forcing frequencies $f \leq 10 \mathrm{~Hz}$. The experimental findings are well described with the numerical results obtained with the same parameters for $\eta, \sigma$ and $\chi$ as in 


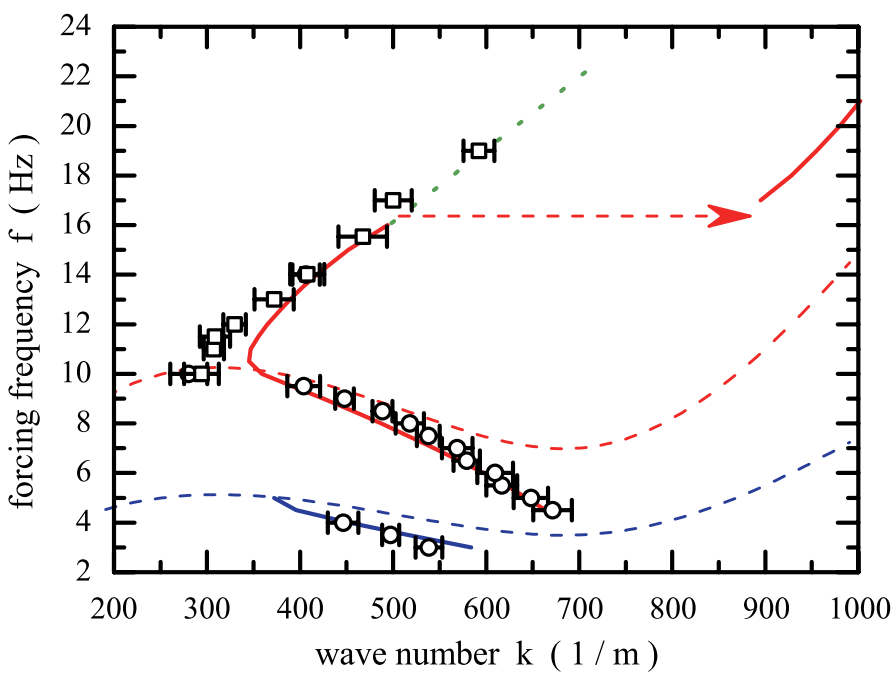

Fig. 26. Forcing frequency $f$ versus wave number $k$ as in Fig. 24 for $M=0.987 M_{\mathrm{C}}^{\mathrm{R}}$. Open circles denote experimental data on the anomalous dispersion branch, while open squares refer to the normal dispersion branch. The dotted line is a guide for the eye, following the subharmonic-dispersion relation. The arrow is explained in the text. Full lines show critical values as in Fig. 24; dashed curves refer to the idealized case $\eta=0$.

the experiments at $M=0$ and $M<\tilde{M}$. For forcing frequencies $f \geq 16 \mathrm{~Hz}$ the numerical results predict a jump in wave number from $k \approx 500 \mathrm{~m}^{-1}$ to $k \approx 900 \mathrm{~m}^{-1}$ as indicated by the arrow in Fig. 26. However, this jump does not appear in the experiment. The data points rather follow the continuation of the low-frequency dispersion branch (as indicated by the dotted line in Fig. 26 as a guide for the eye). This finding is up to now not fully clear, although the topology of the neutral stability curves could explain this result: for frequencies $f \geq 16 \mathrm{~Hz}$ and $\varepsilon_{A} \equiv a / a_{\mathrm{C}}-1=0.05$ the band of possible wave numbers $k$ is about one order of magnitude larger than for driving frequencies within the range of the anomalous dispersion relation. Since the experiments have to be performed at finite supercritical $\varepsilon_{A}$ their exists the possibility for the system to select a wave number different from $k_{\mathrm{C}}$.

\section{Convection in colloidal suspensions}

Convection in a ferrofluid layer heated from below (Rayleigh-Bénard convection) and exposed to a homogeneous external magnetic field in vertical direction has been considered for a long time [78]. But only later [79] it was understood that the binary nature of the ferrofluid as a mixture of carrier liquid and magnetic particles is important to understand the convection properties. 
Ferrofluids, as well as colloidal mixtures in general, differ greatly from molecular mixtures like ethanol-water normally studied in the framework of Rayleigh-Bénard convection. Firstly, the large particle diameter in the nanometer range leads to a very slow concentration diffusion in colloidal mixtures. Secondly, colloids are known to show a very strong Soret effect, i.e., a very strong driving of concentration currents by thermal gradients [80]. This feature has also been explained by the large particle diameter [81]. As a consequence, the solutal diffusion coefficient and the Soret coefficient in ferrofluids and other colloids differ by orders of magnitude from those found in common molecular mixtures. We have investigated this parameter region numerically [82] which has hardly been studied before [83, 84].

\subsection{The system and numerical details}

The system we have considered is a fluid layer confined between two infinitely extended, rigid, and impermeable parallel plates perpendicular to the direction of gravity. The plates are kept at constant temperatures, the lower plate being the warmer one, allowing for buoyancy-driven instabilities. In the conductive ground state without fluid motion, the temperature field thus shows a linear profile in the vertical direction. The fluid is a binary mixture with a nonvanishing Soret effect which couples the temperature into the concentration field dynamics. In particular, in the ground state the Soret effect will establish a linear profile in the concentration field too. The concentration couples back into the dynamics of the other fields via the concentration dependence of the density and thus of the buoyancy force.

For the system of equations governing the system we refer to $[85,86]$. Four dimensionless parameters appear in this system. The Prandtl number $\sigma$ is the ratio of momentum to thermal diffusivity. Typical values for liquids are of the order of 10, and as long as $\sigma \gg 1$, the system is not very sensitive to the exact value. We used $\sigma=10$ for all our calculations. The Rayleigh number $R$ is the control parameter, the dimensionless temperature difference between lower and upper plate. Below we will mainly use the reduced Rayleigh number $r=R / R_{\mathrm{c}}^{0}, R_{\mathrm{c}}^{0}=1707.76$ being the critical Rayleigh number for the pure fluid. The Lewis number $L$ is the ratio of solutal to thermal diffusivity. For molecular mixtures a typical value is $L=0.01$. Colloids on the other hand can easily reach values of $\mathcal{O}\left(10^{-4}\right)$ and smaller. The separation ratio $\psi$ is the ratio of concentration- and temperature-induced density gradients in the ground state. $\psi$ expresses the strength and direction of the Soret effect. In ethanol-water one finds values between -0.5 and 0.5 , depending on mean temperature and concentration. In colloids on the other hand values of $\mathcal{O}(10)$ and larger have been found [80]. Below, we will briefly refer to mixtures with $\sigma=\mathcal{O}(10), L=\mathcal{O}\left(10^{-2}\right)$, and $|\psi|=\mathcal{O}(0.1)$ as liquid molecular mixtures, and to mixtures with $\sigma=\mathcal{O}(10), L=\mathcal{O}\left(10^{-4}\right)$ or less, and $|\psi|>1$ as colloidal mixtures. 
The patterns we have investigated are those that are the most important ones in liquid molecular mixtures. For negative $\psi$ we have investigated stationary and traveling convection roll patterns (traveling waves, TWs). For positive $\psi$ stationary rolls, squares, and crossrolls were investigated. Squares can be thought as superpositions of two perpendicular roll sets of equal amplitude. Crossrolls also consist of perpendicular roll sets, but of unequal amplitude, such that the full square symmetry is not fulfilled. Two different types of crossrolls exist, a stationary one, and an oscillatory variant where the amplitudes are time-dependent.

We used the Galerkin method to compute the convection patterns. Within this method spatially periodic fields are expanded into a complete set of orthonormal functions (modes) using an ansatz $\sim \cos (l k x) \cos (m k y)$ in lateral direction. The basic equations are then projected onto these functions, transforming the system of partial differential equations into an ordinary system of first order in time, with the time-dependent amplitudes as new unknowns. The system is in principle infinite but truncated to allow for a numerical treatment.

\subsection{Linear stability, convection at small amplitudes}

When $\psi=0$, concentration variations will only appear as transients, as without Soret effect the concentration field will always equilibrate. In that case, the ground state becomes unstable at the critical temperature difference $r=1$ of the pure fluid. When the Soret effect is positive, $\psi>0$, the lighter component will go into the direction of higher temperatures, further destabilizing the ground state, and thus convection sets in at smaller $R$ than $R_{\mathrm{c}}^{0}$. For a negative Soret effect $(\psi<0)$ the opposite is the case. The critical $R$ is larger than $R_{\mathrm{c}}^{0}$, and except for very small $|\psi|$ that are not of interest here the first bifurcation out of the ground state is backwards leading to unstable small amplitude convection. Furthermore, the critical Rayleigh number quickly diverges when $|\psi|$ grows. Arbitrary small amplitude convection thus does not exist at all for colloids, and we can ignore negative $\psi$ here.

For $\psi>0$ the first instability is always stationary. For the reduced Rayleigh number of the threshold $r_{\text {stab }}(k)$ the power law dependence

$$
r_{\text {stab }}(k, L, \psi)=h(k) \frac{L}{\psi}
$$

on $L$ and $\psi$ holds for a broad range of $\psi$ and $L$ including the relevant parameters for colloids. This behavior can be seen in Fig. 27 where $r_{\text {stab }}$ is plotted as function of $L$ and $\psi$ for $k=0$, the critical wave number for typical colloids, and $k_{\mathrm{c}}^{0}=3.116$, the critical wave number for pure fluids. This behavior can also be confirmed by examining the semianalytical results for the ground state stability found in [87]. The convective threshold in colloids thus lies very low compared to molecular mixtures. 

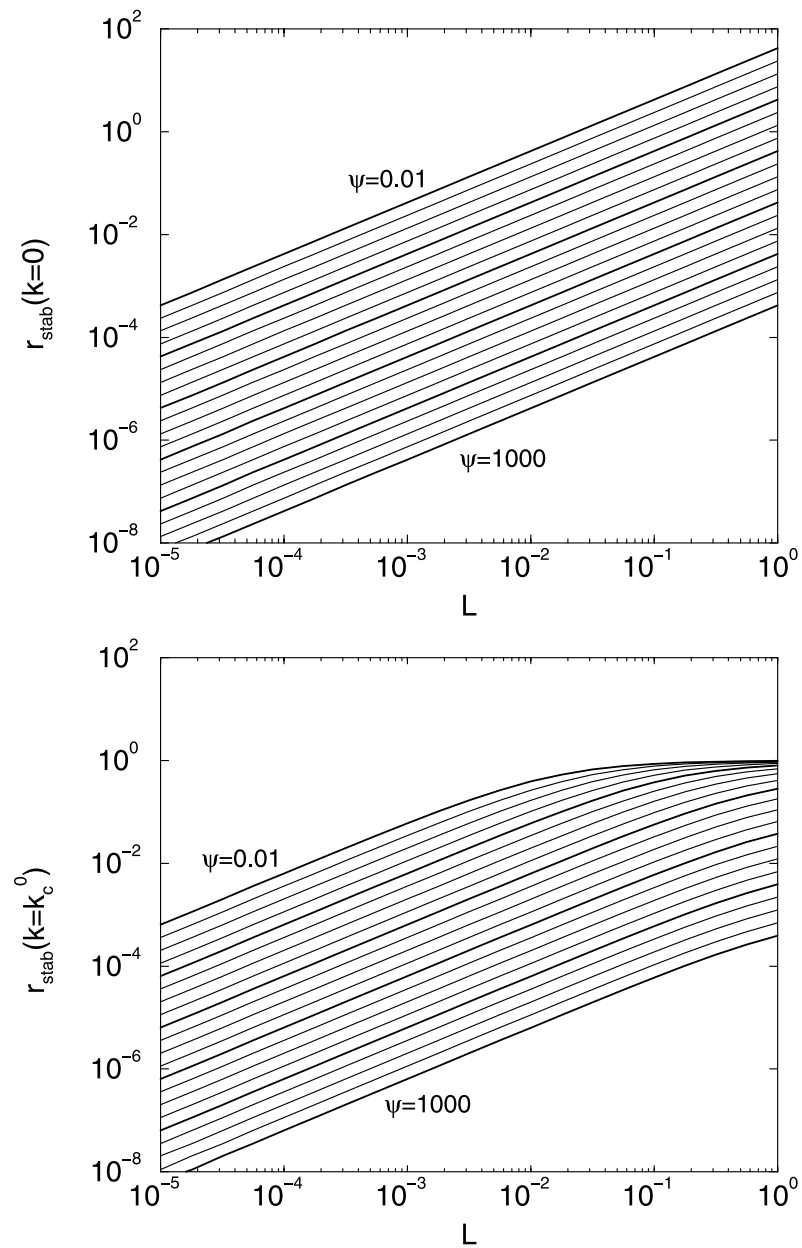

Fig. 27. Reduced stability thresholds of the conductive state, $r_{\text {stab }}(k, \psi, L)$, against non oscillatory convection with wave numbers $k=0$ (top) and $k=k_{\mathrm{c}}^{0}$ (bottom) versus $L$. Between thick lines $\psi$ changes by a factor of 10 .

That the threshold decreases with growing $\psi$ can be explained by the destabilization due to the growing density gradient. In particular, $r_{\text {stab }}$ is for $\psi>0$ always smaller than for pure fluids. The dependence on $L$ can be qualitatively explained by the fact that a larger $L$ leads to faster equilibration of concentration perturbations and thus to increased stability.

A power law does also hold for the initial slope $S$, that can be defined as

$$
S=\frac{\partial N}{\partial r} \quad \text { at } \quad r=r_{\text {stab }},
$$




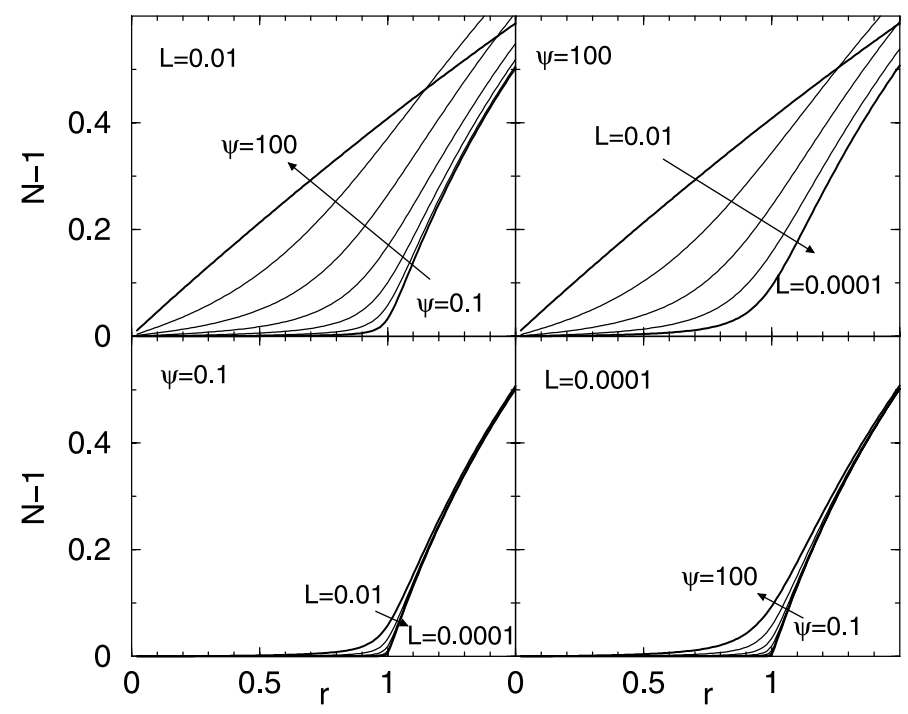

Fig. 28. Nusselt number versus $r$ for $\sigma=10, k=k_{\mathrm{c}}^{0}$, and several $(L, \psi)$-combinations. The parameter that is varied changes by a factor of $\sqrt{10}$ between the curves in each plot.

where $N$ is the Nusselt number, the ratio of total to conductive heat transport through the system. Here one finds $S \sim L \psi$ for typical colloidal as well as for typical molecular mixtures. The proportionality factor depends also on $k$ and $\sigma$. The dependence on $\sigma$ is very weak as long as $\sigma \gg 1$. The type of pattern does also play a role, but for the two relevant patterns that bifurcate out of the ground state, namely rolls and squares, the difference in the slope is practically constant for colloids and moreover only about $2 \%$.

\subsection{Bifurcation scenario for positive $\psi$}

\subsubsection{Bifurcation curves}

Away from the bifurcation point two characteristic convection regimes exist in liquid molecular mixtures that can also be found in colloids, the Soret and Rayleigh regime. These two regimes are visible in the bifurcation diagrams, like in Fig. 28. There we have plotted the Nusselt number $N$ against the reduced Rayleigh number $r$ for roll patterns at $k=k_{\mathrm{c}}^{0}$. Square patterns also exist for all parameter combinations shown but the difference in the Nusselt number between these two patterns is very small, not only near the onset as discussed above but in the whole $r$-interval displayed. Except for the highest $\psi$ and $L$, a sharp bend in the curves separates the Soret region $r<1$ and the Rayleigh region $r>1$, especially when both $L$ and $\psi$ are small. Here, the Nusselt number is quantitatively very similar to that of a pure fluid. In particular, $N \approx 1$ in 
the Soret region, where in a pure fluid the conductive state is still stable and therefore the total heat transport equals the conductive heat transport $(N \equiv 1)$.

The explanation for this behavior is as follows: For small $\psi$ the solutal contribution to the density gradient in the ground state is negligible. Nevertheless, for small $L$ the onset of convection lies significantly lower than for pure fluids because the very slow concentration diffusion allows perturbations in the concentration field to grow before they are diffused away. On the other hand, the slow diffusion makes advective mixing very efficient such that only very small convection amplitudes are needed to reach a stationary state for $r<1$, especially since the concentration gradients are small in the first place. At $r>1$, however, the density gradient becomes strong enough to allow perturbations to grow even in absence of a concentration gradient. The convection amplitude now quickly grows, assuming values typical for pure fluids, while the weak concentration gradients vanish when the mixture becomes homogenized due to advective mixing.

For large $\psi$ and $L$, on the other hand, the solutally induced density gradient dominates and solutal diffusion is faster such that the concentration variations are not easily mixed away. The transition between Soret regime and Rayleigh regime is thus much smoother.

The influence of the large $\psi$ and the small $L$ of colloids compensate each other to a certain degree. A detailed inspection has shown that the bifurcation curves depend mainly on the product $L \psi$. Although no strict power law holds as it does near to the onset, changing $L$ and $\psi$ while keeping the product constant changes the curves only very slightly. Colloids therefore show very similar curves $N(r)$ as liquid molecular mixtures with the same $L \psi$, at least when compared on scales $r=\mathcal{O}(1)$ where the onset of convection that scales like $L / \psi$, not $L \psi$, is very close to zero in both cases.

\subsubsection{Pattern selection}

It is known that for small $L$ and large $\psi$ square patterns are the first stable form of convection above the onset. The square patterns lose their stability in the Rayleigh region against oscillatory crossrolls. Therein the amplitudes of the two constituting perpendicular roll sets oscillate in counterphase sweeping through a square state of equal amplitude, such that first one and than the other roll set becomes the dominant one for half a period. Beyond this bifurcation point there is another one on the square branch, where the stationary crossrolls bifurcate. They gain stability after the oscillatory solution vanishes in an entrainment process [88]. The amplitude of the dominant roll set then grows with growing $r$ while the other one shrinks until the crossrolls end up at the roll branch and the rolls now gain stability.

We have calculated the Rayleigh numbers of three bifurcation points for a wide range of $\psi$ and $L$, namely the bifurcation from squares to oscillatory crossrolls, from squares to stationary crossrolls, and from rolls to stationary 


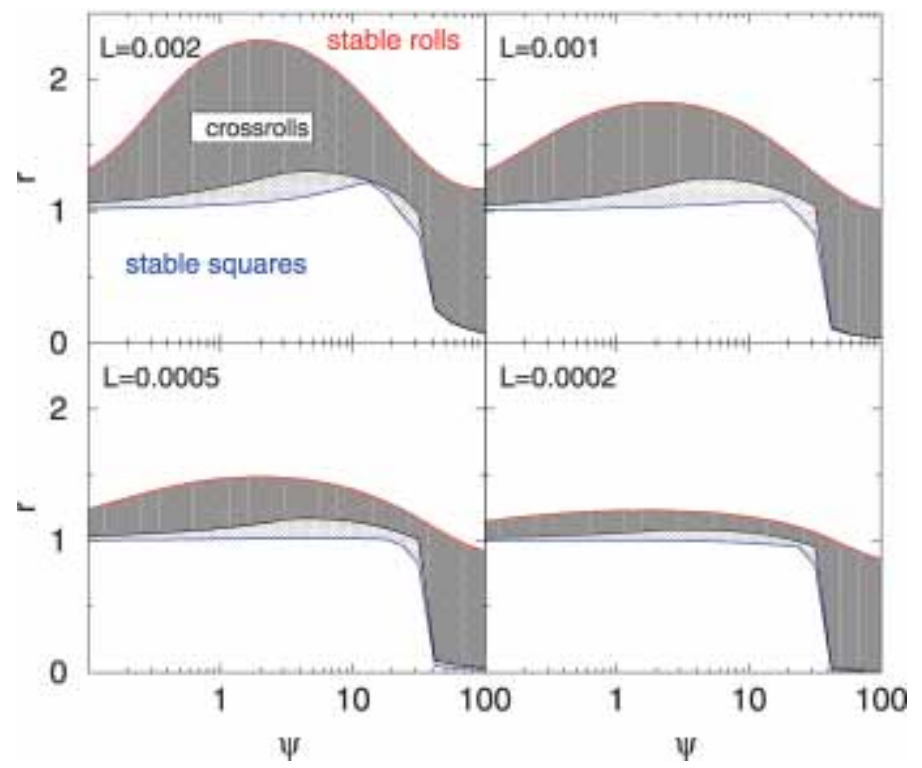

Fig. 29. $r-\psi$-phase diagrams for squares, oscillatory crossrolls, stationary crossrolls, and rolls for $k=k_{\mathrm{c}}^{0}, \sigma=10$, and four different $L$. Rolls are stable in the upper region, squares in the lower. Crossrolls exist in the gray regions.

crossrolls. The resulting phase diagrams for $k=k_{\mathrm{c}}^{0}$ and $\sigma=10$ are shown in Fig. 29. For up to $\psi \approx 40$ we always found the pattern sequence of squares, oscillatory crossrolls, stationary crossrolls, and rolls. Crossrolls can be found in the gray areas. Note however that the boundary between the light and dark gray area marks the bifurcation point from squares to stationary crossrolls. The latter exist in the whole dark gray area but will probably still be unstable towards oscillatory crossrolls in the lower part. The smaller $L$ the smaller the crossroll region becomes: For $L=0.0002$ squares are stable in practically the whole Soret region, rolls in the Rayleigh region with a very small transition region above $r=1$.

For $\psi>40$ the behavior changes abruptly. Squares are still stable directly above onset but lose their stability very quickly. Crossrolls dominate almost the whole Soret region instead. In the Rayleigh region rolls remain stable.

\subsection{Bifurcation scenario for negative $\psi$}

In a wide parameter region typical for molecular mixtures at negative $\psi$ the first instability of the ground state will be an oscillatory one, leading to a pattern of traveling waves (TWs) [89] and related structures. Appearing at a heating rate $r_{\text {osc }}$ the TWs bifurcate backwards, gain stability at a saddle node, and finally end up at another bifurcation point that connects their branch to the station- 


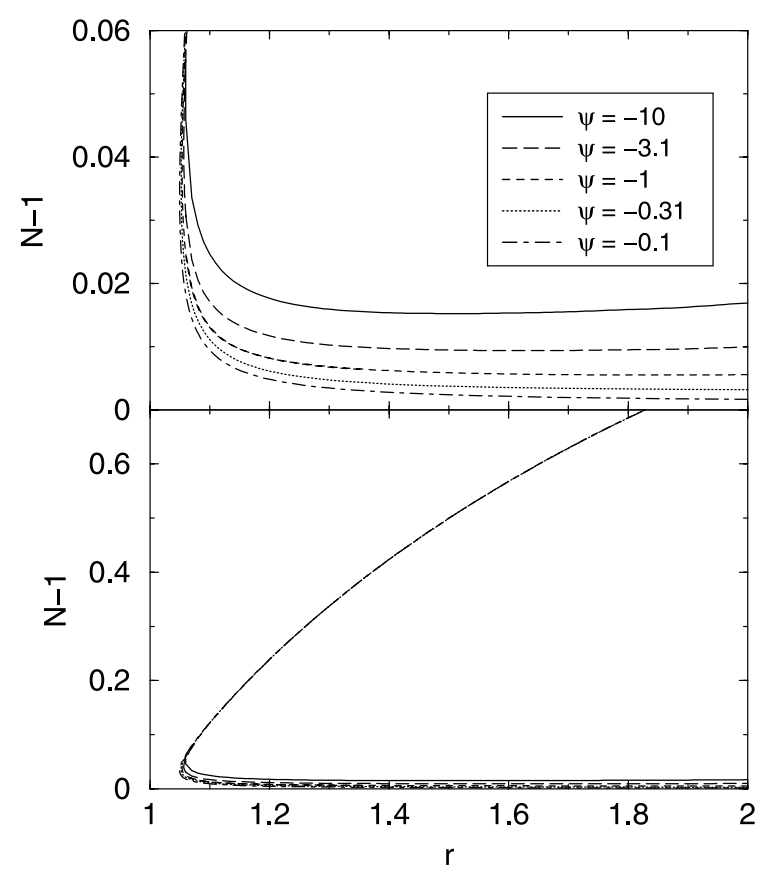

Fig. 30. Bifurcation diagram of Nusselt number versus $r$ of stationary rolls for $\sigma=10$, $k=k_{\mathrm{c}}^{0}$, and different negative $\psi$ such that $|L \psi|=10^{-4}$. Top plot is a magnified part of the bottom plot.

ary rolls branch at the heating rate $r^{*}$. The rolls that have been unstable before gain stability here. For $\psi<-1$ the stabilizing effect of the concentration gradient overcompensates the destabilizing effect of the thermal gradient and neither a stationary nor an oscillatory threshold exists at finite $r$. Although the ground state is always stable convection at larger amplitudes is in principle still possible however, even for colloid parameters $\psi \ll-1$, as long as the advection is strong enough to sufficiently equilibrate the concentration field such that its stabilizing effect vanishes. Again, the mixing can be expected to be more effective when $L$ is small.

Figure 30 shows the stationary roll branches for a fixed product $L \psi=$ $-10^{-4}$. Although both parameters span over two orders of magnitude the Nusselt number is almost independent of the product $L \psi$ on the upper branch, demonstrating again the compensating effect of increasing $|\psi|$ while making $L$ smaller at the same time. Only the lower branch shows some variations as can be seen better in the magnified top plot.

So convection in the form of stationary rolls is possible at moderate $r$ as Fig. 30 demonstrates, but we found that these structures are not stable. The reason lies in the divergence of the lower stability boundary $r^{*}$. It is known that $r^{*}$ 


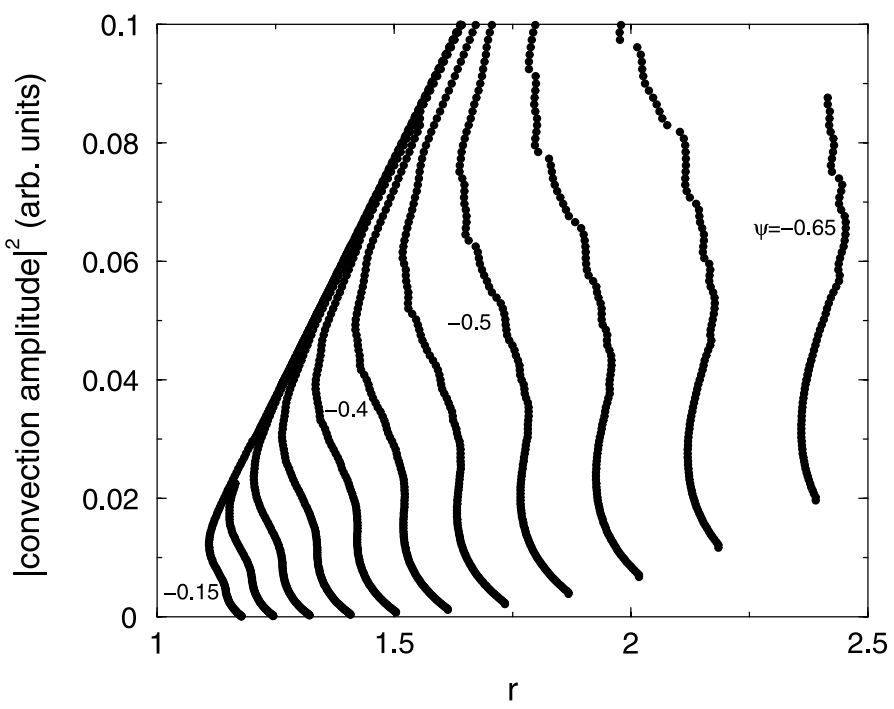

Fig. 31. Bifurcation diagrams of squared TW convection amplitudes versus $r$ for $\sigma=10$, $k=k_{\mathrm{c}}^{0}, L \psi=-10^{-4}$, and several values of $\psi$ between -0.15 and -0.65 .

diverges already for moderate $L$ when $|\psi|$ is increased. For $L=0.01$ for example this is the case at $\psi \approx-0.45$. In this case, reducing $L$ in parallel does not compensate the effect. On the contrary: $r^{*}$ diverges even earlier for fixed $L \psi=-10^{-4}[82]$.

Not only the bifurcation point between TWs and rolls is shifted towards higher heating rates, the whole TW branch moves to the right when $|\psi|$ is increased, and here too the reduction of $L$ does not compensate this behavior. This can be seen in the last plot Fig. 31. Already at $\psi<-0.65$, still not unusual for molecular mixtures, no TWs can be found for $r<2$ when $L \psi=-10^{-4}$.

To conclude: We have numerically studied the bifurcation properties of Rayleigh-Bénard convection in a parameter region far away from what is accessible with commonly studied molecular mixtures but easily accessible with colloidal mixtures. Both, for positive as well as for negative $\psi$ the bifurcation curves for rolls do not change much going from molecular mixtures to colloids, as long as the product $L \psi$ is kept constant.

For colloids with positive $\psi$ we found the pattern sequence of squares, oscillatory crossrolls, stationary crossrolls, and rolls that are known already from the molecular liquid case. For not too large $\psi$ the $r$ interval where crossrolls are found becomes very small in the limit $L \rightarrow 0$. The region $r<1$ and $r>1$ are dominated by squares and rolls respectively. For $\psi$ larger than about 40 , however, squares exist as stable form of convection only immediately above onset. The region $r<1$ is dominated by crossrolls instead. 
For $\psi<0$, we found only the ground state to be stable for typical colloidal parameters. Of the two relevant structures for molecular fluids only the roll branch still exists for moderate $r$, but is unstable. TWs don't exist at all for not too large $r$.

\section{Acknowledgement}

This work was supported by the Deutsche Forschungsgemeinschaft (SFB 277) and by INTAS (03-51-6064). We gratefully acknowledge the contributions of B. Fischer, R. Hempelmann, A. V. Kityk, K. Knorr, C. E. Krill III, S. May, F. Meyer, B. Müller, H. W. Müller, H. Natter, C. Wagner, S. Wiegand as coauthors of different parts of the work reviewed here and discussions with them.

\section{References}

1. B. Huke and M. Lücke, Rep. Prog. Phys. 67 (2004) 1731.

2. B. Huke and M. Lücke, Phys. Rev. E 62 (2000) 6875.

3. B. Huke and M. Lücke, Magnetohydrodynamics 37 (2001) 222.

4. B. Huke and M. Lücke, J. Magn. Magn. Mater. 252 (2002) 132.

5. B. Huke and M. Lücke, Phys. Rev. E 67 (2003) 051403.

6. V. I. Kalikmanov, Physica A 183 (1992) 25.

7. Y. A. Buyevich and A. O. Ivanov, Physica A 190 (1992) 276.

8. P. Debye, Phys. Z. 13 (1912) 97.

9. K. I. Morozov and A. V. Lebedev, J. Magn. Magn. Mater. 85 (1990) 51.

10. A. F. Pshenichnikov, V. V. Mekhonoshin, and A. V. Lebedev, J. Magn. Magn. Mater. 161 (1996) 94.

11. G. A. van Ewijk, G. J. Vroege, and A. P. Philipse, J. Phys.: Condens. Matter 14 (2002) 4915.

12. P. T. Cummings and L. Blum, J. Chem. Phys. 85 (1986) 6658.

13. T. Sato, T. Iijima, M. Seki, and N. Inagaki, J. Magn. Magn. Mater. 65 (1987) 252.

14. A. O. Ivanov and O. B. Kuznetsova, Phys. Rev. E 64 (2001) 041405.

15. R. E. Rosensweig, Ferrohydrodynamics. Cambridge University Press, Cambridge (1985).

16. J. Embs, H. W. Müller, M. Lücke, and K. Knorr, Magnetohydrodynamics 36 (2000) 387.

17. J. Embs, H. W. Müller, C. E. Krill, F. Meyer, H. Natter, B. Müller, S. Wiegand, M. Lücke, R. Hempelmann, and K. Knorr, Magnetohydrodynamics 37 (2001) 222.

18. J. P. Embs, H. W. Müller, C. E. Krill, F. Meyer, H. Natter, B. Müller, S. Wiegand, M. Lücke, R. Hemplemann, and K. Knorr, Z. Phys. Chem. 220 (2006) 153.

19. B. Huke and M. Lücke, Phys. Rev. E 62 (2000) 6875.

20. J. D. Jackson, Klassische Elektrodynamik. de Gruyter, Berlin (1982).

21. T. Weser and K. Stierstadt, Z. Phys. B 59 (1985) 257.

22. H. P. Klug and L. E. Alexander, X-Ray Diffraction Procedures for Polycrystalline and Amorphous Materials. Wiley, New York (1975).

23. B. E. Warren and B. L. Averbach, J. Appl. Phys. 23 (1952) 497.

24. B. E. Warren, X-Ray Diffraction. Dover Publications Inc., Dover, New York (1990).

25. C. E. Krill and R. Birringer, Philos. Mag. A 77 (1998) 621. 
26. H. Natter, M. Schmelzer, M. S. Löffler, C. E. Krill, A. Fitch, and R. Hempelmann, J. Phys. Chem. B 104 (2000) 2467.

27. R. Haberkorn, C. E. Krill, and R. Birringer, Handbook of Nanostructured Materials and Nanotechnology, Vol.2: Spectroscopy and Theory. Academic Press, London (2000) Chap. 3.

28. S. J. Provencher, Comp. Phys. Commun. 27 (1982) 213.

29. J. P. McTague, J. Chem. Phys. 51 (1969) 133.

30. W. F. Hall and S. N. Busenberg, J. Chem. Phys. 51 (1969) 137.

31. M. I. Shliomis, Sov. Phys. JETP 34 (1972) 1291.

32. J. Embs, H. W. Müller, C. Wagner, K. Knorr, and M. Lücke, Phys. Rev. E 61 (2000) R2196.

33. K. Henjes, J. Magn. Magn. Mater. 117 (1992) L311.

34. T. Weser and K. Stierstadt, Z. Phys. B 59 (1985) 253.

35. M. Holderied, L. Schwab, and K. Stierstadt, Z. Phys. B 70 (1988) 431.

36. O. Ambacher, S. Odenbach, and K. Stierstadt, J. Phys. I France 86 (1992) 29.

37. S. Odenbach and H. Gilly, J. Magn. Magn. Mater. 152 (1996) 123.

38. The specifications for the ferrofluid APG933 are as follows: $\mu_{0} M_{\mathrm{s}}=20 \mathrm{mT}$, $\eta=500 \mathrm{mPa}$, density $\rho=1.09 \mathrm{~g} / \mathrm{cm}^{3}, \Phi_{\mathrm{m}}=3.3 \%$, susceptibility $\chi=0.9$, mean diameter of the particle's magnetic core $10 \mathrm{~nm}$, thickness of the polymeric coating $2 \mathrm{~nm}$.

39. J. T. Waldron, Y. P. Kalmykov, and W. T. Coffey, Phys. Rev. E 49 (1994) 3976.

40. One arrives at relation (12) also from the phenomenological low-frequency equations given in Ref. [90]. Also the relaxation equation $\partial_{t} \boldsymbol{M}=-\frac{1}{\tau_{\mathrm{B}}}\left(\boldsymbol{M}-\boldsymbol{M}_{\mathrm{eq}}\right)+$ $\frac{1}{2}(\nabla \times \boldsymbol{v}) \times \boldsymbol{M}+\frac{\mu_{0}}{6 \eta \phi_{\mathrm{h}}}(\boldsymbol{M} \times \boldsymbol{H}) \times \boldsymbol{M}$ suggested by Shliomis [31] reduces under the approximations done in the text to Eq. (12), however, with $\tau_{\perp}$ being replaced by $\tau_{\perp}^{S}=\tau_{\mathrm{B}} /\left(1+\frac{1}{2} \alpha_{0} \mathcal{L}_{0}\right)$. We prefer to fit our data with the field dependence of $\tau_{\perp}[39,42]$ that has been derived without a semi-microscopic estimate of the Onsager coefficient, which couples linear and spin-angular momentum [31].

41. M. I. Shliomis, Chem. Eng. Commun. 67 (1988) 275.

42. M. A. Martsenyuk, Y. L. Raikher, and M. I. Shliomis, Sov. Phys. JETP 38 (1974) 413.

43. A. Leschhorn and M. Lücke, Z. Phys. Chem. 220 (2006) 89.

44. E. Blums, A. Cebers, and M. M. Maiorov, Magnetic Fluids. Walter de Gruyter, Berlin (1997).

45. S. Odenbach, Lect. Notes Phys. m71 (2002).

46. S. Odenbach (Ed.), Lect. Notes Phys. 594 (2002).

47. J. P. Embs, S. May, C. Wagner, A. V. Kityk, A. Leschhorn, and M. Lücke, Phys. Rev. E 73 (2006) 036302.

48. A. Leschhorn and M. Lücke, Z. Phys. Chem. 220 (2006) 219.

49. B. U. Felderhof and H. J. Kroh, J. Chem. Phys. 110 (1999) 7403.

50. B. U. Felderhof, Phys. Rev. E 62 (2000) 3848.

51. M. I. Shliomis, Phys. Rev. E 64 (2001) 060501.

52. H. W. Müller and M. Liu, Phys. Rev. E 64 (2001) 061405.

53. A. Leschhorn and M. Lücke, J. Phys.: Condens. Matter 18 (2006) 2633.

54. Minimizing

$$
\sigma=\sum_{i=1}^{K}\left[M^{\mathrm{eq}}\left(H_{k}\right)-M_{k}\right]^{2}
$$

with respect to $w_{i}$, in which $\left\{H_{k}, M_{k}\right\}_{k=1}^{K}$ indicate the experimental data, leads to an ill-posed problem, resulting in large positive and negative magnetic weights $w_{i}$. To avoid these unphysical results one introduces an additional quantity $\rho=\sum_{i=1}^{N} w_{i}^{2}$ 
and one now minimizes

$$
\sigma^{\prime}=\sigma+\rho \widetilde{\alpha},
$$

with respect to $w_{i}$ where $\widetilde{\alpha}$ refers to the so-called regularization parameter $(\widetilde{\alpha}=0$ leads to the initial ill-posed problem). Using a small but finite positive $\tilde{\alpha}$ allows for the computation of the distribution of the magnetic weights $w_{i}$ as function of the particle diameters $d_{i}$.

55. B. U. Felderhof, Phys. Rev. E 64 (2001) 063502.

56. M. I. Shliomis, Phys. Rev. E 64 (2001) 063501.

57. B. U. Felderhof, Phys. Rev. E 64 (2001) 021508.

58. T. K. McNab, R. A. Fox, and J. F. Boyle, J. Appl. Phys. 39 (1968) 5703.

59. P. C. Fannin, P. A. Preov, and S. W. Charles, J. Phys. D 32 (1999) 1583.

60. P. C. Fannin, J. Magn. Magn. Mater. 136 (1994) 49.

61. J. Embs, C. Wagner, K. Knorr, and M. Lücke, Europhys. Lett. 78 (2007) 44003.

62. M. D. Cowley and R. E. Rosensweig, J. Fluid Mech. 30 (1967) 671.

63. B. Abou, G. Néron de Surgy, and J. E. Wesfreid, Phys. Rev. E 7 (1997) 1159.

64. J. Weilepp and H. R. Brand, J. Phys. II (Paris) 6 (1996) 419.

65. D. Salin, Europhys. Lett. 21 (1993) 667.

66. A. Lange, Magnetohydrodynamics 39 (2003) 65.

67. T. Mahr, A. Groismann, and I. Rehberg, J. Magn. Magn. Mater. 159 (1996) L45.

68. H. W. Müller, Phys. Rev. E 58 (1998) 6199.

69. F. Pétrélis, É. Falcon, and S. Fauve, Eur. Phys. J. B 15 (2000) 3.

70. T. Mahr and I. Rehberg, Phys. Rev. Lett. 81 (1998) 89.

71. T. Mahr and I. Rehberg, Europhys. Lett. 43 (1998) 23.

72. J. Browaeys, J.-C. Bacri, C. Flament, S. Neveu, and R. Perzynski, Eur. Phys. J. B 9 (1999) 335

73. K. Kumar and L. S. Tuckerman, J. Fluid Mech. 279 (1994) 49.

74. P. Huber, V. P. Soprunyuk, J. P. Embs, C. Wagner, M. Deutsch, and S. Kumar, Phys. Rev. Lett. 94 (2005) 184504.

75. C. Wagner, H. W. Müller, and K. Knorr, Phys. Rev. E 68 (2003) 066204.

76. H. W. Müller, H. Wittmer, C. Wagner, J. Albers, and K. Knorr, Phys. Rev. Lett. 78 (1997) 2357.

77. C. Wagner, H. W. Müller, and K. Knorr, Phys. Rev. Lett. 83 (1999) 308.

78. S. Chandrasekhar, Hydrodynamic and Hydromagnetic Stability. Dover Publications Inc., Dover, New York (1981).

79. P. J. Stiles, M. Kagan, and P. J. Blennerhassett, J. Magn. Magn. Mater. 168 (1994) 545.

80. E. Blums, S. Odenbach, A. Mezulis, and M. Maiorov, Phys. Fluids. 10 (1998) 2155.

81. K. I. Morozov, Lect. Notes Phys. 584 (2002) 38.

82. B. Huke, H. Pleiner, and M. Lücke, Phys. Rev. E 75 (2007) 036203.

83. A. Ryskin, H.-W. Müller, and H. Pleiner, Phys. Rev. E 67 (2003) 046302.

84. A. Ryskin and H. Pleiner, Phys. Rev. E 71 (2005) 056303.

85. J. K. Platten and J. C. Legros, Convection in Liquids. Springer, New York (1984).

86. M. Lücke W. Hort, and S. J. Linz, Phys. Rev. A 45 (1992) 3737.

87. St. Hollinger and M. Lücke, Phys. Rev. E 52 (1995) 642.

88. Ch. Jung, B. Huke, and M. Lücke, Phys. Rev. Lett. 81 (1998) 3651.

89. R. W. Walden, P. Kolodner, A. Passner, and C. M. Surko, Phys. Rev. Lett. 55 (1985) 496.

90. V. G. Bashtovoy, B. M. Berkovsky, and A. Vislovich, Introduction to Thermomechanics od Magnetic Fluids. Hemisphere Publishing Corporation, Washington (1988). 